\title{
Rh(III)-Catalyzed C-H Activation-Initiated Directed Cyclopropanation of Allylic Alcohols
}

Supporting Information

Erik J.T. Phipps and Tomislav Rovis*

Table of Contents

I General Methods 2

II General Procedures for the Synthesis of Starting Materials 3

III General Procedure for the Cyclopropanation Reaction and 8 Characterization of Products

$\begin{array}{ll}\text { IV Mechanistic Experiments } & 20\end{array}$

$\begin{array}{lll}\text { V Model for Diastereoselectivity } & 30\end{array}$

V X-ray Crystallographic Data 31

$\begin{array}{lll}\text { VI NMR Spectra } & 33\end{array}$

$\begin{array}{llr}\text { VII References } & 62\end{array}$ 


\section{General Methods}

All reactions were carried out in oven-dried glassware with magnetic stirring. ACS grade TFE and reagents were purchased from TCI, Strem, Alfa Aesar, and Sigma-Aldrich and were used without further purification. Dichloromethane, tetrahydrofuran, diethyl ether were degassed with argon and passed through two columns of neutral alumina. Column chromatography was performed on SiliCycle $\AA$ SilicaFlash $\AA$ P60, 40-63 $\mu \mathrm{m} 60 \AA$ and in general were run using flash techniques. ${ }^{1}$ Thin layer chromatography was performed on SiliCycle $\AA 250 \mu \mathrm{m} 60 \AA$ plates. Visualization was accomplished with UV light $(254 \mathrm{~nm}) .{ }^{1} \mathrm{H},{ }^{19} \mathrm{~F}$, and ${ }^{13} \mathrm{C}$ NMR spectra were collected at ambient temperature in $\mathrm{CDCl}_{3}$ on Bruker $300 \mathrm{~Hz}, 400 \mathrm{MHz}$, or $500 \mathrm{MHz}$ spectrometers. Chemical shifts are expressed as parts per million $(\delta, \mathrm{ppm})$ and are referenced to the residual solvent peak of chloroform $\left({ }^{1} \mathrm{H}=7.26 \mathrm{ppm} ;{ }^{13} \mathrm{C}=77.2 \mathrm{ppm}\right)$. Scalar coupling constants $(J)$ are quoted in Hz. Multiplicity is reported as follows: $\mathrm{s}=$ singlet, $\mathrm{d}=$ doublet, $\mathrm{t}=$ triplet, $\mathrm{q}=$ quartet, $\mathrm{m}=$ multiplet). Mass spectra were obtained on a Waters (LRMS). Infrared (IR) spectra were obtained with neat samples on a Bruker Tensor 27 FT-IR spectrometer with OPUS software. Typically, the experiment consisted measuring the transmission in 16 scans in the region from 4000 to $400 \mathrm{~cm}^{-1}$. 


\section{General Procedure for Starting Materials}

A. Synthesis of $\left[\mathrm{Cp}^{* \mathrm{CF}^{3}} \mathrm{RhCl}_{2}\right]_{2}$ Catalyst $^{2}$

Synthesis of 1,2,3,4-tetramethyl-5-(trifluoromethyl)cyclopenta-1,3-diene (+ isomers)

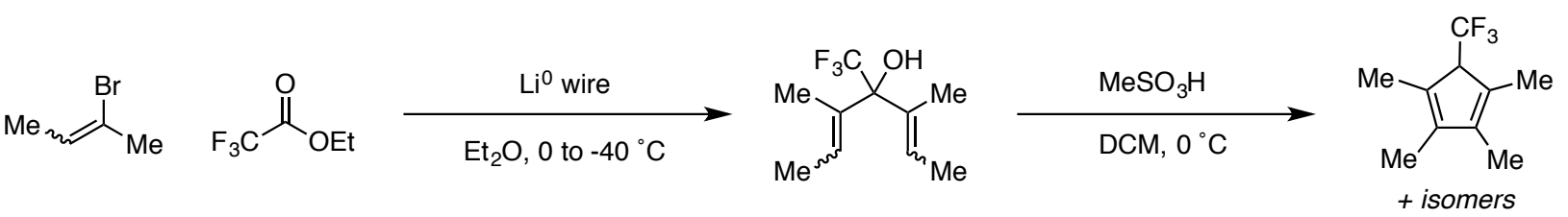

Figure 1.

Following a reported procedure, Li wire (1.291 g, $186 \mathrm{mmol}, 4$ equiv.) was cut into $\sim 5 \mathrm{~mm}$ size pieces and added to $\mathrm{Et}_{2} \mathrm{O}(2.85 \mathrm{M}, 69 \mathrm{~mL})$ in a 250-mL 3-neck flask with a magnetic stir bar and cooled to $0{ }^{\circ} \mathrm{C}$ in an ice bath. 2-bromo-2-butene (cis + trans) (9.7 mL, $95.3 \mathrm{mmol}, 2.05$ equiv.) diluted with $10 \mathrm{~mL} \mathrm{Et}_{2} \mathrm{O}$ was added dropwise over 10 minutes. The heterogeneous mixture was stirred for 2 hours then cooled to $-40{ }^{\circ} \mathrm{C}$ (MeCN, Dry Ice bath). Ethyl trifluoroacetate $(5.4 \mathrm{~mL}$, $46.5 \mathrm{mmol}, 1$ equiv.) diluted with $5 \mathrm{~mL} \mathrm{Et}_{2} \mathrm{O}$ was added dropwise over 10 minutes. The solution was stirred for an additional 90 minutes. The solution was quenched with $20 \mathrm{~mL}$ of $2 \mathrm{M} \mathrm{HCl}$ solution and diluted with $100 \mathrm{~mL}$ DI $\mathrm{H}_{2} \mathrm{O}$. The solution was transferred to a separatory funnel and the layers separated. The aqueous layer was extracted three times with $\mathrm{Et}_{2} \mathrm{O}$. The organic layers were combined and washed with saturated sodium bicarbonate, water, and brine then dried over $\mathrm{Na}_{2} \mathrm{SO}_{4}$ and concentrated. The resulting yellow liquid was vacuum distilled to give the intermediate alcohol, a clear liquid, in $43 \%$ yield ( $4.1734 \mathrm{~g})$.

The intermediate alcohol (1.0 g, $4.8 \mathrm{mmol}, 1$ equiv.) was dissolved in DCM (0.16 M, $30 \mathrm{~mL})$ in a 50-mL flask equipped with a magnetic stir bar and cooled to $0{ }^{\circ} \mathrm{C}$ in an ice bath. Methanesulfonic acid (3.1 mL, $48 \mathrm{mmol}, 10$ equiv.) was quickly added and the solution was stirred for 5 minutes. The resulting dark red solution was then poured into $50 \mathrm{~mL}$ of cooled DI $\mathrm{H}_{2} \mathrm{O}$. The solution was transferred to separatory funnel and the layers were separated. The aqueous layer was extracted three times with DCM. The organic layers were combined and washed with saturated sodium bicarbonate, dried over dried over $\mathrm{Na}_{2} \mathrm{SO}_{4}$ and concentrated. $\mathrm{HCp}^{* \mathrm{CF} 3}$ (+ isomers) was purified by flash chromatography (Hexanes) and afforded in 69\% yield $(1.3374 \mathrm{~g})$

Synthesis of $\left[\mathrm{Cp}^{* \mathrm{CF}^{3}} \mathrm{RhCl}_{2}\right]_{2}$

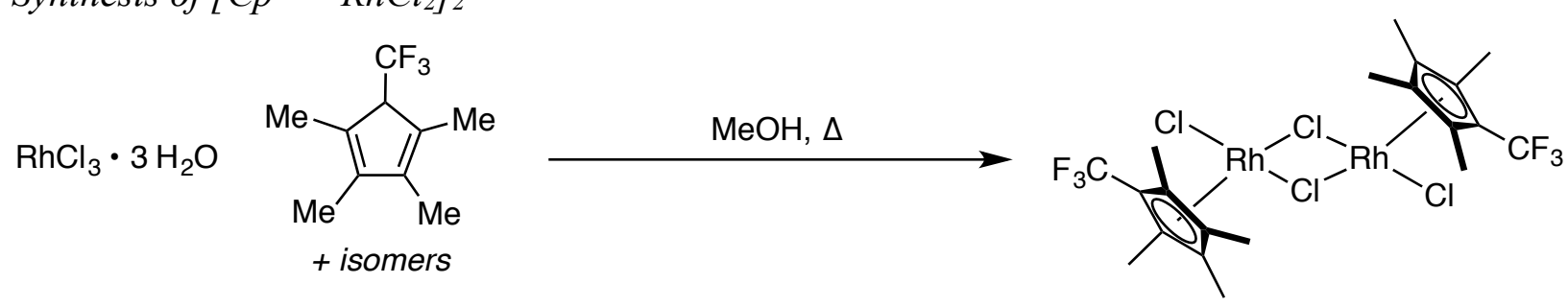

Figure 2.

From a reported procedure, in a $250-\mathrm{mL}$ flask equipped with a magnetic stir bar and a reflux condenser under $\mathrm{N}_{2}$ atmosphere was added $\mathrm{RhCl}_{3} \cdot 3 \mathrm{H}_{2} \mathrm{O}$ (700 mg, $2.6 \mathrm{mmol}, 1$ equiv.), $\mathrm{MeOH}$ (140 mL, 0.019 M), and $\mathrm{HCp}^{* \mathrm{CF} 3}$ (1.3374 g, $7.28 \mathrm{mmol}, 2.7$ equiv.). The solution was refluxed under $\mathrm{N}_{2}$ atmosphere for 3 days where a dark red precipitate was visible on the sides of the flask. The reaction was cooled to $0{ }^{\circ} \mathrm{C}$ in an ice bath and the precipitate was filtered and washed with 
EtOH two additional times. The resulting red solid was collected and dried to afford $72 \%$ yield $(1.33 \mathrm{~g})$. The

\section{B. Synthesis of N-enoxyphthalimide Substrates}

Method $\mathrm{A}^{3}$

Synthesis of (1,2-dibromoethyl)arenes

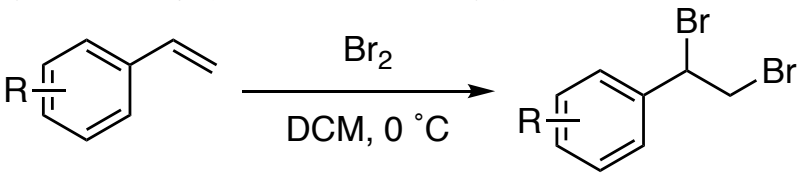

Figure3.

Styrene (1 equiv.) in DCM $(0.5 \mathrm{M})$ was cooled to $0{ }^{\circ} \mathrm{C}$ and $\mathrm{Br}_{2}$ (1.2 equiv.) was added via syringe and stirred at $0{ }^{\circ} \mathrm{C}$ for $\sim 1$ hour. The solution was quenched with sat. $\mathrm{Na}_{2} \mathrm{~S}_{2} \mathrm{O}_{3}$ until the solution became colorless. The resulting solutions was then filtered through a pad a celite ${ }^{\circledR}$ and washed with DCM. The layers were then separated and the aqueous layer was extracted with DCM. The combined organic layers were then washed with brine, dried over $\mathrm{Na}_{2} \mathrm{SO}_{4}$, and concentrated. The resulting white solid was directly carried on without purification.

Synthesis of $\alpha$-bromostyrenes

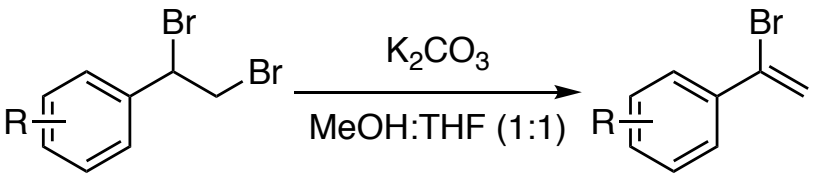

Figure 4.

(1,2-dibromoethyl)arenes (1 equiv.) was stirred in a $0.25 \mathrm{M}$ solution of $1: 1$ methanol and THF at room temperature. Potassium carbonate ( 2 equiv.) was added and the solution stirred until the reaction was judged complete by TLC ( $\sim 3 \mathrm{hrs}$.). The reaction was then quenched with D.I. water and the volatiles were removed. The resulting aqueous layer was extracted with ether and the combined organic layers were then washed with brine, dried over $\mathrm{Na}_{2} \mathrm{SO}_{4}$, and concentrated. The resulting oil was directly carried on without purification.

Synthesis of (1-arylvinyl)boronic acids

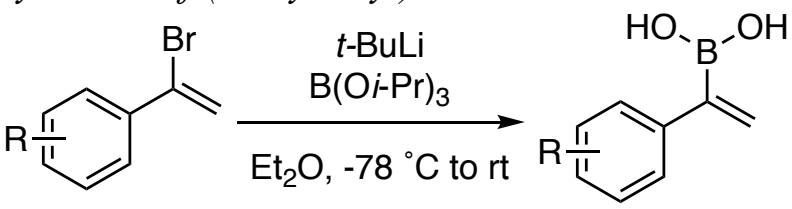

Figure 5.

$\alpha$-bromostyrene in dry diethyl ether was put under inert atmosphere in a 2-neck flask and cooled to $-78{ }^{\circ} \mathrm{C}$. A $1.7 \mathrm{M}$ solution of $\mathrm{t}-\mathrm{BuLi}$ in pentanes $(2.1$ equiv.) was added dropwise and the solution was stirred at $-78{ }^{\circ} \mathrm{C}$ for 30 minutes. Tri-isopropylborate (1.2 equiv.) was added dropwise to the solution over 30 minutes. After the addition was complete, the solution was stirred at $-78{ }^{\circ} \mathrm{C}$ for 2 hours after which the solution was removed from the cold bath and stirred at room temperature overnight. To the resulting yellow-orange solution was added $1 \mathrm{M} \mathrm{HCl}$ solution and was stirred for 2 hours. The layers were separated and the aqueous layer was extracted with ether. The combined organic layers were then washed with $1 \mathrm{M} \mathrm{NaOH}$ solution and the layers were separated. The aqueous layer was acidified to $\mathrm{pH} \approx 1$ and extracted with ethyl 
acetate. The combined organic layers were then washed with brine, dried over $\mathrm{Na}_{2} \mathrm{SO}_{4}$, and concentrated. The crude product was directly carried on without purification.

Synthesis of $N$-enoxyphthalimides

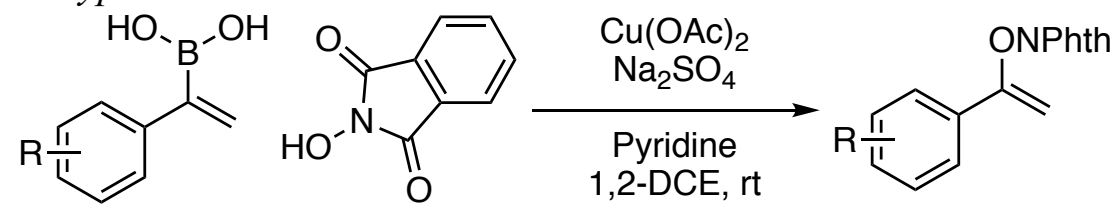

Figure 6.

Boronic acid ( 2 equiv.), copper(II) acetate (1 equiv.), $N$-hydroxyphthalimide (1 equiv.), and anhydrous sodium sulfate (4 equiv.) were combined in a flask and diluted with 1,2dichloroethane to form a $0.1 \mathrm{M}$ solution of $N$-hydroxyphthalimide. Pyridine (3 equiv.) was added via syringe and the solution was stirred at room temperature open to air for 2 days. At the end of the stirring period, the volatiles were removed and the resulting solids were purified by column chromatography. The purified solids were then used in the cyclopropanation reactions. 
Method B ${ }^{4}$

Figure 7.

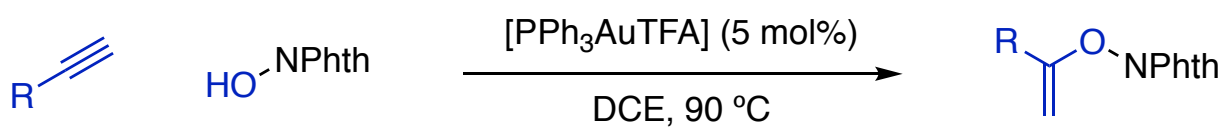

Following a reported procedure, alkyne (3 equiv.), $N$-hydroxphthalimide (1 equiv.), and Au catalyst $(5 \mathrm{~mol} \%)$ were combined in a 1.5 dram vial in the glove box under Ar and dissolved in 1,2-DCE (0.2M). The vial was sealed and removed from the glovebox and placed in an aluminum heating block overnight at $90{ }^{\circ} \mathrm{C}$. The reaction was then cooled to room temperature, diluted with DCM and passed through a pad of Celite ${ }^{\circledR}$. The solvent was removed and the crude residue was purified by column chromatography (19:1, Hex:EtOAc).

Compounds Synthesized by Method A<smiles>C=C(O[PbH])c1ccccc1</smiles><smiles>C=C(ONc1ccccc1)c1cccc2ccccc12</smiles><smiles>C=C(O[PbH])c1ccccc1F</smiles><smiles>C=C(ONc1ccccc1)c1ccc(C)cc1</smiles><smiles>C=C(ONc1ccccc1)c1ccc(C(C)(C)C)cc1</smiles><smiles>C=C(O[PbH])c1ccc(F)cc1</smiles><smiles>C=C(O[Nb]c1ccccc1)c1cccc(C)c1</smiles><smiles>C=C(O[PbH]c1ccccc1)c1cccc(F)c1</smiles><smiles></smiles>

Compounds Synthesized by Method B<smiles>C=C(O[PbH]c1ccccc1)c1ccc(OC)cc1</smiles><smiles>C=C(CBr)O[NbH2+]</smiles>

Figure 8.

The spectra of known compounds were consistent with literature precedent. 
C. Synthesis of Allylic Alcohol Substrates and Analogs.

Allylic alcohols were purchased from commercial suppliers unless noted below:<smiles>O=C/C=C/P=O</smiles><smiles>[Y][W]</smiles><smiles>COCCOC</smiles><smiles>[R]C(O)/C=C/P</smiles>

$$
X=\mathrm{Cl} \text { or Br }
$$

$$
R=C y \text { or } P h
$$

Figure 9.

This procedure was performed according to literature precedent. ${ }^{5,6,7}$
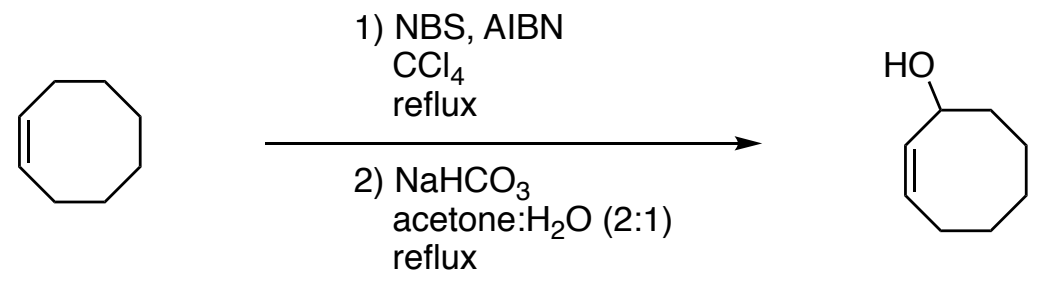

Figure 10.

This procedure was performed similar to literature precedent. ${ }^{8}$ The crude mixture was purified by flash chromatography $(9: 1 \rightarrow 4: 1$, Hex:EtOAc).
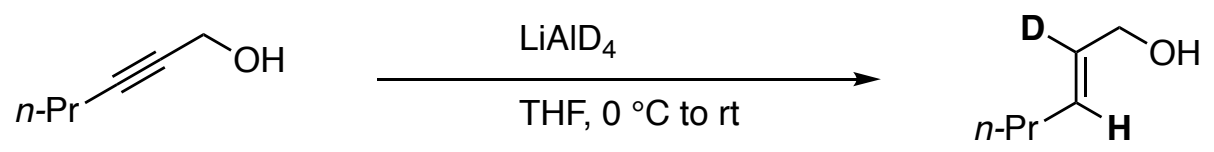

Figure 11.

This procedure was performed according to literature precedent. ${ }^{9}$<smiles>OC/C=C/P</smiles>

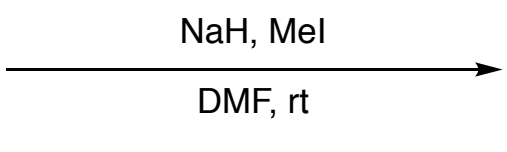<smiles>COCC=CC(C)C</smiles>

Figure 12.

This procedure was performed according to literature precedent. ${ }^{10}$

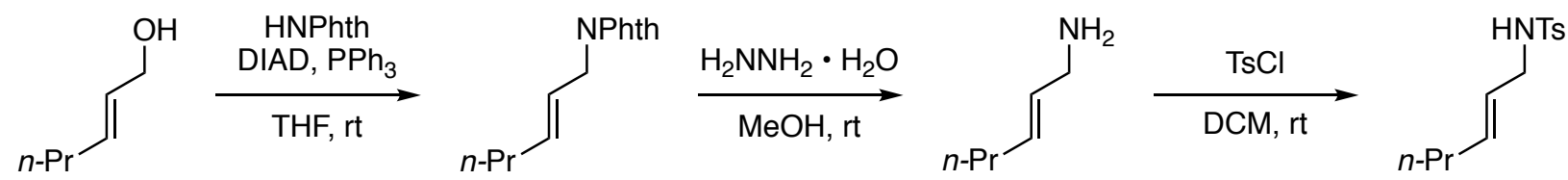

Figure 13.

This procedure was performed according to the literature precedent. ${ }^{11}$ 

Products<smiles>C=C(ONc1ccccc1)c1ccccc1</smiles><smiles>[R]C([R])=C([R])O</smiles>

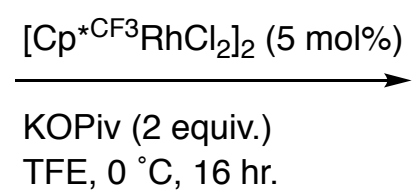<smiles>[R]C(O)C1([R])[C@H](C(=O)c2ccccc2)[C@@]1([R])[2H]</smiles>

Figure 14.

$N$-enoxyphthalimide $(0.12 \mathrm{mmol})$, catalyst $\left[\mathrm{Cp}^{* \mathrm{CF}^{3}} \mathrm{RhCl}_{2}\right]_{2}(5 \mathrm{~mol} \%, 0.006 \mathrm{mmol}, 4.4 \mathrm{mg})$, and KOPiv (2 equiv., $0.24 \mathrm{mmol}, 33.6 \mathrm{mg}$ ) were weighed in a 1-dram vial with a magnetic stirbar. Cooled TFE $(0.2 \mathrm{M}, 600 \mu \mathrm{L})$ was added followed by allylic alcohol (1.2 equiv., $0.144 \mathrm{mmol})$. The vial was sealed with a screw-cap and placed in an aluminium block cooled to $0{ }^{\circ} \mathrm{C}$ surrounded by ice in an insulated box and stirred for 16 hours. Upon completion judged by TLC, TFE was removed by rotary evaporation and the residue was taken up in EtOAc and filtered through a silica plug flushing with EtOAc. The filtrate was concentrated to $\sim 1 \mathrm{~mL}$ and transferred to a 1.5-dram vial where the solution was partitioned with the addition of $10 \% \mathrm{NaOH}$ solution. The aqueous layer was extracted three times with EtOAc and the combined organic extracts were filtered through a pad of celite ${ }^{\circledR}$ and $\mathrm{Na}_{2} \mathrm{SO}_{4}$ then concentrated. The crude residue was purified by flash chromatography (Hexane:EtOAc, 19:1 $\rightarrow 9: 1 \rightarrow 4: 1$ ) to afford the cyclopropane product.

3aa 2-(hydroxymethyl)-3-propylcyclopropyl)(phenyl)methanone

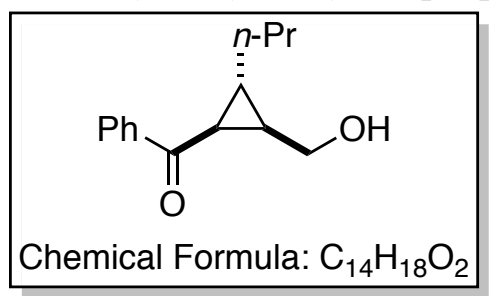

$\mathrm{Y}=81 \%$. Yellow oil. $\mathrm{R}_{\mathrm{f}}=0.22(4: 1$ Hexanes:EtOAc)

${ }^{1}$ H NMR (500 MHz, Chloroform- $d$ ) $\delta 8.05-7.94(\mathrm{~m}, 2 \mathrm{H}), 7.56(\mathrm{t}, J=7.4 \mathrm{~Hz}, 1 \mathrm{H}), 7.47(\mathrm{t}, J=$ $7.6 \mathrm{~Hz}, 2 \mathrm{H}), 3.95(\mathrm{dd}, J=12.0,4.7 \mathrm{~Hz}, 1 \mathrm{H}), 3.76(\mathrm{dd}, J=12.0,8.3 \mathrm{~Hz}, 1 \mathrm{H}), 2.55$ (dd, $J=8.4$, $5.0 \mathrm{~Hz}, 1 \mathrm{H}), 2.14(\mathrm{~s}, 1 \mathrm{H}), 1.89-1.81(\mathrm{~m}, 1 \mathrm{H}), 1.78-1.71(\mathrm{~m}, 1 \mathrm{H}), 1.45(\mathrm{tdd}, J=13.4,10.7,4.8$ $\mathrm{Hz}, 4 \mathrm{H}), 0.93(\mathrm{t}, J=6.9 \mathrm{~Hz}, 3 \mathrm{H})$.

${ }^{13}$ C NMR $\left(126 \mathrm{MHz}, \mathrm{CDCl}_{3}\right) \delta 200.2,138.6,133.0,128.7,128.2,60.0,35.6,35.4,30.3,28.5$, $22.4,14.0$.

IR(neat) 3456, 2924, 1660, 1453, 1228, 1019, $700 \mathrm{~cm}^{-1}$

LRMS m/z (ESI APCI) calculated for $\mathrm{C}_{14} \mathrm{H}_{18} \mathrm{O}_{2}[\mathrm{M}+\mathrm{H}] 219.1$, found 219.1. 
3ab 2-(hydroxymethyl)-3-propylcyclopropyl)(phenyl)methanone

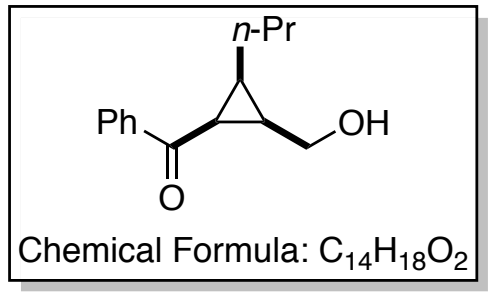

$\mathrm{Y}=62 \%$. Pale-yellow oil. $\mathrm{R}_{\mathrm{f}}=0.22(4: 1$ Hexanes:EtOAc)

${ }^{1}$ H NMR $(500 \mathrm{MHz}$, Chloroform- $d$ ) $\delta 8.03-7.95(\mathrm{~m}, 2 \mathrm{H}), 7.61-7.54(\mathrm{~m}, 1 \mathrm{H}), 7.46(\mathrm{dd}, J=$ $8.4,7.0 \mathrm{~Hz}, 2 \mathrm{H}), 4.06(\mathrm{dd}, J=7.9,2.7 \mathrm{~Hz}, 2 \mathrm{H}), 2.73(\mathrm{dd}, J=9.4,7.9 \mathrm{~Hz}, 1 \mathrm{H}), 2.45(\mathrm{~s}, 1 \mathrm{H}), 1.90$ - $1.72(\mathrm{~m}, 2 \mathrm{H}), 1.55$ (ddt, $J=13.9,8.4,6.9 \mathrm{~Hz}, 1 \mathrm{H}), 1.49-1.40(\mathrm{~m}, 1 \mathrm{H}), 1.35-1.25(\mathrm{~m}, 2 \mathrm{H})$, $0.85(\mathrm{t}, J=7.4 \mathrm{~Hz}, 3 \mathrm{H})$.

${ }^{13}$ C NMR $\left(126 \mathrm{MHz}, \mathrm{CDCl}_{3}\right) \delta 200.7,138.9,133.1,128.7,128.3,58.7,28.0,28.0,26.5,25.5$, 23.1, 14.0.

IR(neat) 3433, 2957, 1680, 1449, 1209, 1020, $699 \mathrm{~cm}^{-1}$

LRMS m/z (ESI APCI) calculated for $\mathrm{C}_{14} \mathrm{H}_{18} \mathrm{O}_{2}[\mathrm{M}+\mathrm{H}] 2$ 219.1, found 219.1 .

3ba 2-(hydroxymethyl)-3-propylcyclopropyl)(p-tolyl)methanone

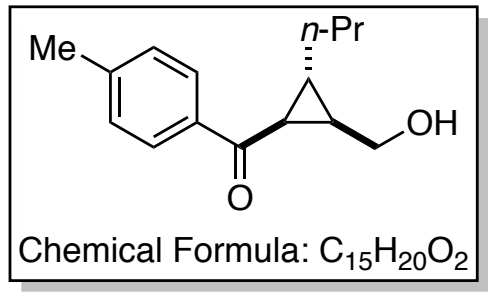

$\mathrm{Y}=72 \%$. Yellow oil. $\mathrm{R}_{\mathrm{f}}=0.22$ (4:1 Hexanes:EtOAc)

${ }^{1}$ H NMR $(500 \mathrm{MHz}$, Chloroform- $d) \delta 7.90(\mathrm{~d}, J=8.2 \mathrm{~Hz}, 2 \mathrm{H}), 7.27(\mathrm{~d}, J=7.5 \mathrm{~Hz}, 2 \mathrm{H}), 3.94$ $(\mathrm{dd}, J=12.3,4.8 \mathrm{~Hz}, 1 \mathrm{H}), 3.80-3.71(\mathrm{dd}, J=9.0,5.8 \mathrm{~Hz}, 1 \mathrm{H}), 2.52(\mathrm{dd}, J=8.4,5.1 \mathrm{~Hz}, 1 \mathrm{H})$, $2.42(\mathrm{~s}, 3 \mathrm{H}), 2.19(\mathrm{~s}, 1 \mathrm{H}), 1.86-1.79(\mathrm{~m}, 1 \mathrm{H}), 1.72(\mathrm{tdd}, J=8.3,6.5,4.6 \mathrm{~Hz}, 1 \mathrm{H}), 1.52-1.37$ (m, 4H), $0.98-0.89$ (t, $J=7.0 \mathrm{~Hz}, 3 \mathrm{H})$.

${ }^{13}$ C NMR $\left(126 \mathrm{MHz}, \mathrm{CDCl}_{3}\right) \delta 199.8,143.8,136.1,129.4,128.4,60.1,35.5,35.4,30.1,28.3$, 22.4, 21.8, 14.0.

IR(neat) $3441,2957,2923,1663,1607,1454,1233,1179,103,665 \mathrm{~cm}^{-1}$

LRMS m/z (ESI APCI) calculated for $\mathrm{C}_{15} \mathrm{H}_{20} \mathrm{O}_{2}[\mathrm{M}+\mathrm{H}]$ 233.2, found 233.2. 
3ca (4-(tert-butyl)phenyl)-2-(hydroxymethyl)-3-propylcyclopropyl)methanone

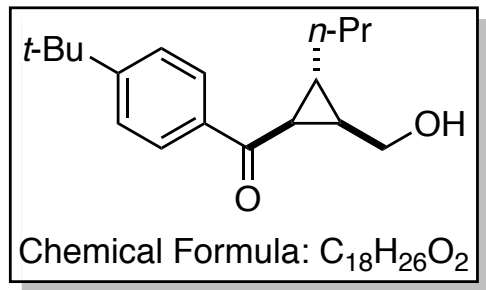

$\mathrm{Y}=76 \%$. Pale-yellow oil. $\mathrm{R}_{\mathrm{f}}=0.24$ (4:1, Hexanes:EtOAc).

${ }^{1}$ H NMR (500 MHz, Chloroform- $d$ ) $\delta 7.95(\mathrm{~d}, J=8.5 \mathrm{~Hz}, 1 \mathrm{H}), 7.49$ (d, $\left.J=8.5 \mathrm{~Hz}, 2 \mathrm{H}\right), 3.98-$ $3.91(\mathrm{~m}, 1 \mathrm{H}), 3.81-3.72(\mathrm{~m}, 1 \mathrm{H}), 2.54(\mathrm{dd}, J=8.4,5.0 \mathrm{~Hz}, 1 \mathrm{H}), 2.18(\mathrm{~s}, 1 \mathrm{H}), 1.87-1.80(\mathrm{~m}$, $1 \mathrm{H}), 1.73$ (tdd, $J=8.3,6.5,4.6 \mathrm{~Hz}, 1 \mathrm{H}), 1.53-1.40$ (m, 4H), 1.35 (s, 9H), 0.93 (t, $J=6.9 \mathrm{~Hz}$, $3 \mathrm{H})$.

${ }^{13} \mathrm{C}$ NMR $\left(126 \mathrm{MHz}, \mathrm{CDCl}_{3}\right){ }^{13} \mathrm{C} \mathrm{NMR}\left(126 \mathrm{MHz}, \mathrm{CDCl}_{3}\right) \delta 199.9,156.8,134.5,128.2,125.7$, $123.8,60.12,35.5,35.4,31.3,30.2,28.3,22.4,14.0$.

IR(neat) $3210,2956,2923,2852,1736,1606,1234,1109,834,852,796 \mathrm{~cm}^{-1}$

LRMS m/z (ESI APCI) calculated for $\mathrm{C}_{18} \mathrm{H}_{26} \mathrm{O}_{2}[\mathrm{M}+\mathrm{H}]$ 275.2, found 275.2.

3da (4-fluorophenyl)(2-(hydroxymethyl)-3-propylcyclopropyl)methanone

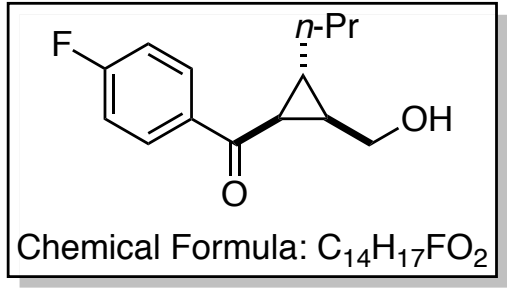

$\mathrm{Y}=69 \%$. Yellow Oil. $\mathrm{R}_{\mathrm{f}}=0.16(4: 1$ Hexanes:EtOAc).

${ }^{1}$ H NMR ${ }^{1} \mathrm{H}$ NMR $(500 \mathrm{MHz}$, Chloroform- $d$ ) $\delta 8.02(\mathrm{dd}, J=8.5,5.4 \mathrm{~Hz}, 2 \mathrm{H}), 7.13(\mathrm{t}, J=8.5$ $\mathrm{Hz}, 2 \mathrm{H}), 3.94(\mathrm{dd}, J=11.9,4.7 \mathrm{~Hz}, 1 \mathrm{H}), 3.72(\mathrm{dd}, J=12.0,8.4 \mathrm{~Hz}, 1 \mathrm{H}), 2.48(\mathrm{dd}, J=8.4,5.0$ $\mathrm{Hz}, 1 \mathrm{H}), 2.14(\mathrm{~s}, 1 \mathrm{H}), 1.82(\mathrm{p}, J=6.2 \mathrm{~Hz}, 1 \mathrm{H}), 1.74(\mathrm{qd}, J=8.3,5.6 \mathrm{~Hz}, 1 \mathrm{H}), 1.44(\mathrm{tt}, J=13.9$, $7.1 \mathrm{~Hz}, 4 \mathrm{H}), 0.92(\mathrm{t}, J=6.8 \mathrm{~Hz}, 3 \mathrm{H})$.

${ }^{13}$ C NMR $\left(126 \mathrm{MHz}, \mathrm{CDCl}_{3}\right) \delta 198.5,166.8,164.8,135.0,135.0,130.9,130.8,115.9,115.7$, 60.0, 35.6, 35.4, 30.1, 28.4, 22.4, 14.0.

${ }^{19}$ F NMR (282 MHz, Chloroform- $d$ ) $\delta-104.95$ (ddd, $J=13.7,8.5,5.4 \mathrm{~Hz}$ ).

IR(neat) 3458, 2958, 2926, 1667, 1599, 1507, 1229, 1155, 1031, $838 \mathrm{~cm}^{-1}$

LRMS m/z (ESI APCI) calculated for $\mathrm{C}_{14} \mathrm{H}_{17} \mathrm{FO}_{2}[\mathrm{M}+\mathrm{H}]$ 237.1, found 237.1. 
3ea (2-(hydroxymethyl)-3-propylcyclopropyl)(4-methoxyphenyl)methanone

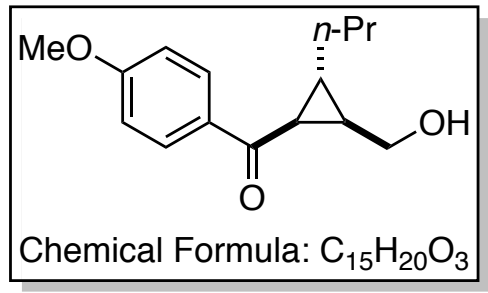

$\mathrm{Y}=77 \%$. Pale-Yellow Oil. $\mathrm{R}_{\mathrm{f}}=0.06$ (4:1 Hexanes:EtOAc).

${ }^{1}$ H NMR (500 MHz, Chloroform- $d$ ) $\delta 7.99(\mathrm{~d}, J=8.8 \mathrm{~Hz}, 2 \mathrm{H}), 6.95(\mathrm{~d}, J=8.8 \mathrm{~Hz}, 2 \mathrm{H}), 3.94$

$(\mathrm{dd}, J=12.0,4.6 \mathrm{~Hz}, 1 \mathrm{H}), 3.87(\mathrm{~s}, 3 \mathrm{H}), 3.75(\mathrm{dd}, J=12.1,8.2 \mathrm{~Hz}, 1 \mathrm{H}), 2.49$ (dd, $J=8.4,5.0 \mathrm{~Hz}$, $1 \mathrm{H}), 2.26(\mathrm{~s}, 1 \mathrm{H}), 1.80(\mathrm{p}, J=6.3 \mathrm{~Hz}, 1 \mathrm{H}), 1.73-1.66(\mathrm{~m}, 1 \mathrm{H}), 1.51-1.38(\mathrm{~m}, 4 \mathrm{H}), 0.93(\mathrm{t}, J=$ $6.9 \mathrm{~Hz}, 3 \mathrm{H})$.

${ }^{13}$ C NMR $\left(126 \mathrm{MHz}, \mathrm{CDCl}_{3}\right) \delta 198.7,131.6,130.5,113.9,60.2,55.7,35.5,35.1,29.9,28.0$, 22.4, 14.0 .

IR(neat) 3436, 2957, 2926, 1655, 1600, 1235, 1170, 1026, $845 \mathrm{~cm}^{-1}$

LRMS m/z (ESI APCI) calculated for $\mathrm{C}_{15} \mathrm{H}_{20} \mathrm{O}_{3}[\mathrm{M}+\mathrm{H}]$ 249.1, found 249.1.

3fa (2-(hydroxymethyl)-3-propylcyclopropyl)(m-tolyl)methanone

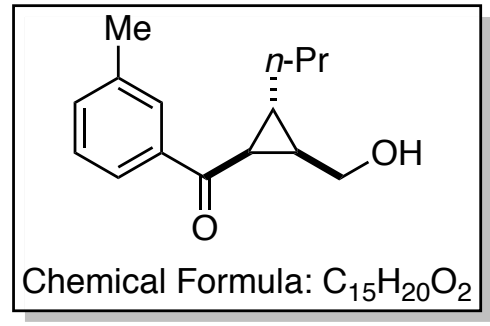

$\mathrm{Y}=52 \%$. Pale-yellow oil. $\mathrm{R}_{\mathrm{f}}=0.30$ (4:1 Hexanes:EtOAc).

${ }^{1}$ H NMR (500 MHz, Chloroform- $d$ ) $\delta 7.80(\mathrm{q}, J=2.4 \mathrm{~Hz}, 2 \mathrm{H}), 7.42-7.32(\mathrm{~m}, 2 \mathrm{H}), 3.94(\mathrm{dd}, J$ $=12.0,4.6 \mathrm{~Hz}, 1 \mathrm{H}), 3.76(\mathrm{dd}, J=12.0,8.3 \mathrm{~Hz}, 1 \mathrm{H}), 2.54(\mathrm{dd}, J=8.4,5.0 \mathrm{~Hz}, 1 \mathrm{H}), 2.42(\mathrm{~s}, 3 \mathrm{H})$, $2.15(\mathrm{~s}, 1 \mathrm{H}), 1.88-1.79(\mathrm{~m}, 1 \mathrm{H}), 1.74(\mathrm{tdd}, J=8.4,6.5,4.6 \mathrm{~Hz}, 1 \mathrm{H}), 1.51-1.38(\mathrm{~m}, 4 \mathrm{H}), 0.97-$ $0.89(\mathrm{~m}, 3 \mathrm{H})$.

${ }^{13}$ C NMR $\left(126 \mathrm{MHz}, \mathrm{CDCl}_{3}\right) \delta 200.3,138.5,138.4,133.6,128.6,128.4,125.3,59.9,35.4,35.3$, 30.1, 28.3, 22.2, 21.4, 13.8 .

IR(neat) 3445, 2955, 2870, 1664, 1604, 1163, 1054, 1030, $708 \mathrm{~cm}^{-1}$

LRMS m/z (ESI APCI) calculated for $\mathrm{C}_{15} \mathrm{H}_{20} \mathrm{O}_{2}[\mathrm{M}+\mathrm{H}]$ 233.2, found 233.2. 
3ga 2-(hydroxymethyl)-3-propylcyclopropyl)(3-methoxyphenyl)methanone

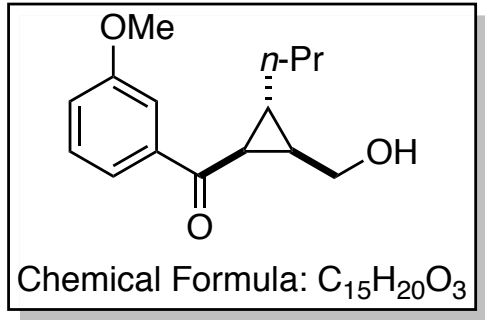

$\mathrm{Y}=93 \%$. Yellow oil. $\mathrm{R}_{\mathrm{f}}=0.18$ (4:1, Hexanes:EtOAc).

${ }^{1}$ H NMR (500 MHz, Chloroform- $d$ ) $\delta 7.60(\mathrm{~d}, J=8.2 \mathrm{~Hz}, 1 \mathrm{H}), 7.49(\mathrm{t}, J=2.1 \mathrm{~Hz}, 1 \mathrm{H}), 7.38(\mathrm{t}, J$ $=7.9 \mathrm{~Hz}, 1 \mathrm{H}), 7.10(\mathrm{dd}, J=8.2,2.7 \mathrm{~Hz}, 1 \mathrm{H}), 3.94(\mathrm{dd}, J=11.9,4.7 \mathrm{~Hz}, 1 \mathrm{H}), 3.85(\mathrm{~s}, 3 \mathrm{H}), 3.75$ $(\mathrm{dd}, J=12.2,8.1 \mathrm{~Hz}, 1 \mathrm{H}), 2.53(\mathrm{dd}, J=8.4,5.0 \mathrm{~Hz}, 1 \mathrm{H}), 2.19(\mathrm{bs}, 1 \mathrm{H}), 1.86-1.79(\mathrm{~m}, 1 \mathrm{H})$, $1.78-1.70(\mathrm{~m}, 1 \mathrm{H}), 1.53-1.37(\mathrm{~m}, 4 \mathrm{H}), 0.96-0.90(\mathrm{~m}, 3 \mathrm{H})$.

${ }^{13}$ C NMR (126 MHz, $\left.\mathrm{CDCl}_{3}\right) \delta 200.0,159.9,139.9,129.7,120.9,119.4,112.5,60.0,55.6,35.6$, 35.4, 30.3, 28.6, 22.3, 13.9 .

IR(neat) 3437, 2957, 2926, 1664, 1586, 1462, 1261, 1034, $778 \mathrm{~cm}^{-1}$

LRMS m/z (ESI APCI) calculated for $\mathrm{C}_{15} \mathrm{H}_{20} \mathrm{O}_{3}[\mathrm{M}+\mathrm{H}] 249.1$, found 249.1.

3ha (3-fluorophenyl)-2-(hydroxymethyl)-3-propylcyclopropyl)methanone

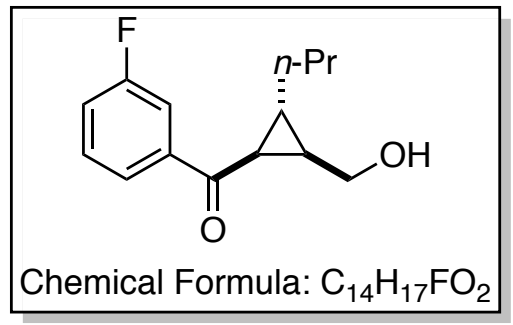

$\mathrm{Y}=54 \%$. Yellow oil. $\mathrm{R}_{\mathrm{f}}=0.15$ (4:1, Hexanes:EtOAc).

${ }^{1}$ H NMR $(500 \mathrm{MHz}$, Chloroform- $d) \delta 7.79(\mathrm{dt}, J=7.8,1.2 \mathrm{~Hz}, 1 \mathrm{H}), 7.66(\mathrm{ddd}, J=9.5,2.6,1.6$ $\mathrm{Hz}, 1 \mathrm{H}), 7.45(\mathrm{td}, J=8.0,5.5 \mathrm{~Hz}, 1 \mathrm{H}), 7.29-7.23(\mathrm{~m}, 2 \mathrm{H}), 3.95(\mathrm{dd}, J=11.9,4.7 \mathrm{~Hz}, 1 \mathrm{H}), 3.73$ $(\mathrm{dd}, J=11.9,8.4 \mathrm{~Hz}, 1 \mathrm{H}), 2.50(\mathrm{dd}, J=8.4,5.0 \mathrm{~Hz}, 1 \mathrm{H}), 1.98(\mathrm{~s}, 1 \mathrm{H}), 1.88-1.82(\mathrm{~m}, 1 \mathrm{H}), 1.78$ (tdd, $J=8.5,6.6,4.7 \mathrm{~Hz}, 1 \mathrm{H}), 1.52-1.38(\mathrm{~m}, 4 \mathrm{H}), 0.98-0.89(\mathrm{~m}, 3 \mathrm{H})$.

${ }^{13}$ C NMR (126 MHz, $\left.\mathrm{CDCl}_{3}\right) \delta 198.8(\mathrm{~d}, J=2.0 \mathrm{~Hz}), 164.0,162.0,130.4$ (d, $\left.J=7.7 \mathrm{~Hz}\right), 124.0$ $(\mathrm{d}, J=3.1 \mathrm{~Hz}), 120.0(\mathrm{~d}, J=21.6 \mathrm{~Hz}), 115.0(\mathrm{~d}, J=22.3 \mathrm{~Hz}), 59.9,35.9,35.34,30.4,28.8,22.4$, 14.0 .

${ }^{19}$ F NMR (282 MHz, Chloroform- $\left.d\right) \delta-111.15(\mathrm{td}, J=9.0,5.7 \mathrm{~Hz})$.

IR(neat) 3439, 2958, 2925, 1670, 1588, 1443, 1252, 1030, $785 \mathrm{~cm}^{-1}$

LRMS m/z (ESI APCI) calculated for $\mathrm{C}_{14} \mathrm{H}_{17} \mathrm{FO}_{2}[\mathrm{M}+\mathrm{H}] 237.1$, found 237.1. 
3ia (2-fluorophenyl)(2-(hydroxymethyl)-3-propylcyclopropyl)methanone

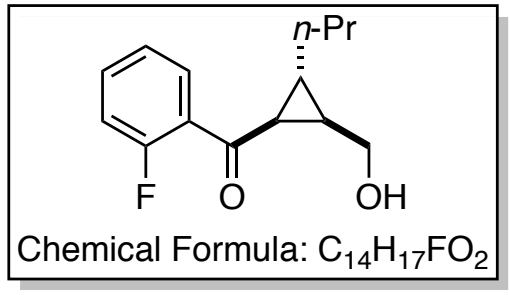

$\mathrm{Y}=44 \%$. Pale-yellow Oil. $\mathrm{R}_{\mathrm{f}}=0.19$ (4:1 Hexanes:EtOAc) .

${ }^{1}$ H NMR (300 MHz, Chloroform- $d$ ) $\delta 7.74$ (td, $J=7.6,1.9 \mathrm{~Hz}, 1 \mathrm{H}$ ), 7.49 (dddd, $J=8.5,7.1,5.0$, $1.9 \mathrm{~Hz}, 1 \mathrm{H}), 7.28-7.07(\mathrm{~m}, 2 \mathrm{H}), 3.96(\mathrm{dd}, J=12.0,4.7 \mathrm{~Hz}, 1 \mathrm{H}), 3.78(\mathrm{t}, J=10.1 \mathrm{~Hz}, 1 \mathrm{H}), 2.54$ (ddd, $J=8.1,5.1,2.7 \mathrm{~Hz}, 1 \mathrm{H}), 1.95(\mathrm{~s}, 1 \mathrm{H}), 1.89$ (ddd, $J=6.5,5.1,1.3 \mathrm{~Hz}, 1 \mathrm{H}), 1.77$ (tdd, $J=$ $8.3,6.6,4.8 \mathrm{~Hz}, 1 \mathrm{H}), 1.51-1.34(\mathrm{~m}, 4 \mathrm{H}), 0.98-0.88(\mathrm{~m}, 3 \mathrm{H})$.

${ }^{13}$ C NMR $\left(126 \mathrm{MHz}, \mathrm{CDCl}_{3}\right) \delta 198.8(\mathrm{~d}, J=3.1 \mathrm{~Hz}), 161.6(\mathrm{~d}, J=255.0 \mathrm{~Hz}), 134.2(\mathrm{~d}, J=9.0$ $\mathrm{Hz}), 130.4(\mathrm{~d}, J=2.6 \mathrm{~Hz}), 128.0(\mathrm{~d}, J=12.6 \mathrm{~Hz}), 124.6(\mathrm{~d}, J=3.6 \mathrm{~Hz}), 116.8(\mathrm{~d}, J=25.1 \mathrm{~Hz})$, $59.9,36.3,35.3,34.4(\mathrm{~d}, J=7.7 \mathrm{~Hz}), 29.6,22.3,14.0$.

${ }^{19} \mathbf{F}$ NMR $\left(282 \mathrm{MHz}, \mathrm{CDCl}_{3}\right) \delta-110.33(\mathrm{dt}, J=8.3,4.0 \mathrm{~Hz})$.

IR(neat) 3213, 2956, 2922, 1653, 1607, 1234, 1109, 1036, $834 \mathrm{~cm}^{-1}$

LRMS m/z (ESI APCI) calculated for $\mathrm{C}_{14} \mathrm{H}_{16} \mathrm{~F}_{2} \mathrm{O}_{2}[\mathrm{M}+\mathrm{H}]$ 237.1, found 237.1.

3ja 2-(hydroxymethyl)-3-propylcyclopropyl)(naphthalen-2-yl)methanone<smiles>O=C(c1ccc2ccccc2c1)C1C(CO)C1CO</smiles>

$\mathrm{Y}=50 \%$. Pale-yellow solid. $\mathrm{R}_{\mathrm{f}}=0.18$ (4:1 Hexanes:EtOAc).

${ }^{1}$ H NMR $(500 \mathrm{MHz}$, Chloroform- $d) \delta 8.55(\mathrm{~d}, J=1.7 \mathrm{~Hz}, 1 \mathrm{H}), 8.05(\mathrm{dd}, J=8.6,1.8 \mathrm{~Hz}, 1 \mathrm{H})$, $7.98(\mathrm{~d}, J=8.1 \mathrm{~Hz}, 1 \mathrm{H}), 7.89$ (dd, $J=10.8,8.3 \mathrm{~Hz}, 2 \mathrm{H}), 7.58$ (dddd, $J=21.9,8.1,6.8,1.3 \mathrm{~Hz}$, 2H), 3.99 (dd, $J=12.0,4.7 \mathrm{~Hz}, 1 \mathrm{H}$ ), 3.80 (dd, $J=12.0,8.3 \mathrm{~Hz}, 1 \mathrm{H}), 2.71$ (dd, $J=8.4,5.0 \mathrm{~Hz}$, $1 \mathrm{H}), 2.14$ (s, $0 \mathrm{H}), 1.96-1.87$ (m, 1H), 1.81 (tdd, $J=8.3,6.6,4.7 \mathrm{~Hz}, 1 \mathrm{H}), 1.50$ (dtd, $J=14.3$, $12.8,11.8,6.9 \mathrm{~Hz}, 4 \mathrm{H}), 0.95(\mathrm{t}, J=7.0 \mathrm{~Hz}, 3 \mathrm{H})$.

${ }^{13}$ C NMR (126 MHz, $\mathrm{CDCl}_{3}$ ) 200.0, 135.9, 135.6, 132.7, 129.8, 129.7, 128.5, 128.5, 127.9, $126.9,124.1,60.1,35.7,35.5,30.3,28.5,22.4,14.0$.

IR(neat) 3300, 2872, 2857, 1657, 1181, 1123, 1046, 1027, 822, $749 \mathrm{~cm}^{-1}$

LRMS m/z (ESI APCI) calculated for $\mathrm{C}_{18} \mathrm{H}_{20} \mathrm{O}_{2}[\mathrm{M}+\mathrm{H}] 269.2$, found 269.2. 
3ka 1-(2-(hydroxymethyl)-3-propylcyclopropyl)-3-phenylpropan-1-one

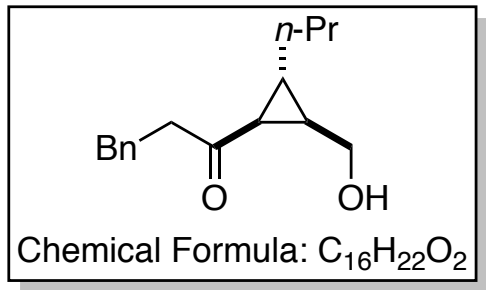

$\mathrm{Y}=92 \%$. Pale-yellow oil. $\mathrm{R}_{\mathrm{f}}=0.21$ (3:1 Hexanes:EtOAc).

${ }^{1}$ H NMR (500 MHz, Chloroform- $d$ ) $\delta 7.31$ - $7.26(\mathrm{~m}, 2 \mathrm{H}), 7.21-7.18(\mathrm{~m}, 3 \mathrm{H}), 3.90-3.80(\mathrm{~m}$, $1 \mathrm{H}), 3.65$ (t, $J=10.2 \mathrm{~Hz}, 1 \mathrm{H}), 3.02-2.88(\mathrm{~m}, 4 \mathrm{H}), 2.01(\mathrm{~s}, 1 \mathrm{H}), 1.83(\mathrm{dd}, J=8.3,5.0 \mathrm{~Hz}, 1 \mathrm{H})$, $1.62(\mathrm{qd}, J=6.6,4.9 \mathrm{~Hz}, 1 \mathrm{H}), 1.53(\mathrm{tdd}, J=8.2,6.6,4.4 \mathrm{~Hz}, 1 \mathrm{H}), 1.42-1.28(\mathrm{~m}, 3 \mathrm{H}), 0.89(\mathrm{t}, J$ $=7.2 \mathrm{~Hz}, 3 \mathrm{H})$.

${ }^{13}$ C NMR $\left(126 \mathrm{MHz}, \mathrm{CDCl}_{3}\right) \delta 210.2,141.2,128.7,128.5,126.3,59.5,46.3,35.3,33.1,30.2$, 28.7, 22.3, 13.9 .

IR(neat) $3386,2958,2925,2872,1689,1454,1378,1034,733,700 \mathrm{~cm}^{-1}$

LRMS m/z (ESI APCI) calculated for $\mathrm{C}_{16} \mathrm{H}_{22} \mathrm{O}_{2}[\mathrm{M}+\mathrm{H}]$ 247.3, found 247.3.

3ac (2-(hydroxymethyl)-3-methylcyclopropyl)(phenyl)methanone

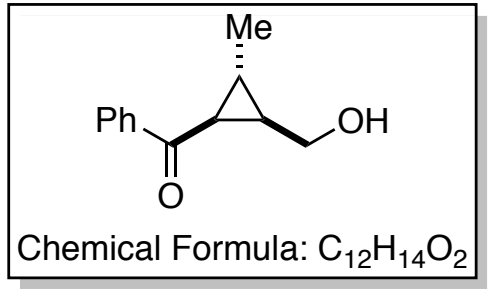

$\mathrm{Y}=89 \%$. Light yellow solid. $\mathrm{R}_{\mathrm{f}}=0.50$ (4:1 Hexanes:EtOAc).

${ }^{1}$ H NMR (500 MHz, Chloroform- $d$ ) $\delta 8.06-7.94(\mathrm{~m}, 2 \mathrm{H}), 7.62-7.52(\mathrm{~m}, 1 \mathrm{H}), 7.47$ (dd, $J=$ 8.3, 7.0 Hz, 2H), $3.96(\mathrm{dd}, J=12.0,4.7 \mathrm{~Hz}, 1 \mathrm{H}), 3.74(\mathrm{dd}, J=12.0,8.3 \mathrm{~Hz}, 1 \mathrm{H}), 2.50(\mathrm{dd}, J=$ 8.4, $5.0 \mathrm{~Hz}, 1 \mathrm{H}), 2.17$ (s, 1H), 1.84 (h, $J=6.0 \mathrm{~Hz}, 1 \mathrm{H}), 1.73$ (tdd, $J=8.3,6.4,4.6 \mathrm{~Hz}, 1 \mathrm{H}), 1.26$ $(\mathrm{d}, J=6.0 \mathrm{~Hz}, 4 \mathrm{H})$.

${ }^{13}$ C NMR $\left(126 \mathrm{MHz}, \mathrm{CDCl}_{3}\right) \delta 200.3,138.6,133.1,128.7,128.3,60.0,36.5,31.5,22.9,18.3$.

IR(neat) 3412, 2955, 2889, 1660, 1598, 1219, 1021, 743, $688 \mathrm{~cm}^{-1}$

LRMS m/z (ESI APCI) calculated for $\mathrm{C}_{12} \mathrm{H}_{14} \mathrm{O}_{2}[\mathrm{M}+\mathrm{H}]$ 191.1, found 191.1. 
3ad (2-(hydroxymethyl)-2-methylcyclopropyl)(phenyl)methanone

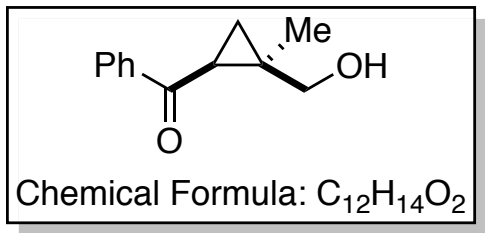

$\mathrm{Y}=62 \%$ Pale-yellow solid. $\mathrm{R}_{\mathrm{f}}=0.19$ (4:1 Hexanes:EtOAc)

${ }^{1}$ H NMR (500 MHz, Chloroform- $d$ ) $\delta 8.02-7.97(\mathrm{~m}, 2 \mathrm{H}), 7.59-7.54(\mathrm{~m}, 1 \mathrm{H}), 7.47(\mathrm{dd}, J=$ 8.4, $7.0 \mathrm{~Hz}, 2 \mathrm{H}), 3.75(\mathrm{dd}, J=11.8,4.6 \mathrm{~Hz}, 1 \mathrm{H}), 3.61(\mathrm{dd}, J=11.8,5.0 \mathrm{~Hz}, 1 \mathrm{H}), 2.54(\mathrm{dd}, J=$ 7.8, $5.7 \mathrm{~Hz}, 1 \mathrm{H}), 1.89$ (t, $J=6.0 \mathrm{~Hz}, 1 \mathrm{H}), 1.64(\mathrm{dd}, J=5.7,4.3 \mathrm{~Hz}, 1 \mathrm{H}), 1.42(\mathrm{~s}, 3 \mathrm{H}), 1.10(\mathrm{dd}, J$ $=7.8,4.3 \mathrm{~Hz}, 1 \mathrm{H})$.

${ }^{13}$ C NMR $\left(126 \mathrm{MHz}, \mathrm{CDCl}_{3}\right) \delta 199.7,138.6,133.0,128.7,128.3,64.7,33.3,31.5,22.8,21.1$. IR(neat) 3439, 2914, 1669, 1269, 1230, 1022, 714

LRMS m/z (ESI APCI) calculated for $\mathrm{C}_{12} \mathrm{H}_{14} \mathrm{O}_{2}[\mathrm{M}+\mathrm{H}]$ 191.1, found 191.1.

3ae (3-(hydroxymethyl)-2,2-dimethylcyclopropyl)(phenyl)methanone

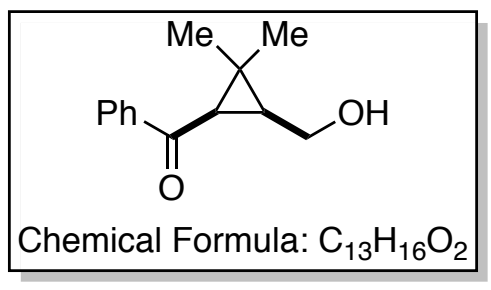

$\mathrm{Y}=82 \%$ Pale-yellow oil. $\mathrm{R}_{\mathrm{f}}=0.18$ (4:1 Hexanes:EtOAc)

${ }^{1}$ H NMR (500 MHz, Chloroform- $d$ ) $\delta 7.98-7.89(\mathrm{~m}, 2 \mathrm{H}), 7.61-7.54(\mathrm{~m}, 1 \mathrm{H}), 7.48(\mathrm{dd}, J=$ $8.4,7.0 \mathrm{~Hz}, 2 \mathrm{H}), 4.10-4.03(\mathrm{~m}, 1 \mathrm{H}), 4.02-3.95(\mathrm{~m}, 1 \mathrm{H}), 2.62(\mathrm{q}, J=3.8 \mathrm{~Hz}, 1 \mathrm{H}), 2.41(\mathrm{~d}, J=$ $7.9 \mathrm{~Hz}, 1 \mathrm{H}), 1.66$ (ddd, $J=9.6,8.0,6.9 \mathrm{~Hz}, 1 \mathrm{H}), 1.41(\mathrm{~s}, 3 \mathrm{H}), 1.11(\mathrm{~s}, 3 \mathrm{H})$.

${ }^{13}$ C NMR $\left(126 \mathrm{MHz}, \mathrm{CDCl}_{3}\right) \delta 200.4,138.8,133.2,128.8,128.3,59.5,35.7,35.6,28.8,28.5$, 15.4 .

IR(neat) 3222, 2911, 1668, 1273, 1220, $692 \mathrm{~cm}^{-1}$

LRMS m/z (ESI APCI) calculated for $\mathrm{C}_{13} \mathrm{H}_{16} \mathrm{O}_{2}[\mathrm{M}+\mathrm{H}]$ 205.1, found 205.1. 
3af (3-(hydroxymethyl)-2-methyl-2-(4-methylpent-3-en-1-yl)cyclopropyl)(phenyl)methanone

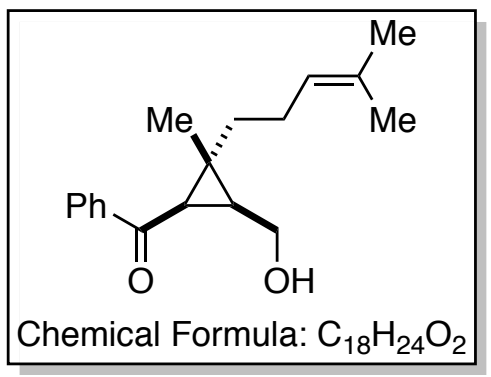

$\mathrm{Y}=55 \%$. Colorless oil. $\mathrm{R}_{\mathrm{f}}=0.24$ (4:1 Hexanes:EtOAc).

${ }^{1}$ H NMR (Major) (500 MHz, Chloroform- $d$ ) $\delta 7.99-7.89(\mathrm{~m}, 2 \mathrm{H}), 7.61-7.51(\mathrm{~m}, 1 \mathrm{H}), 7.46$ (dd, $J=8.5,7.0 \mathrm{~Hz}, 2 \mathrm{H}), 5.13$ (tt, $J=6.9,1.5 \mathrm{~Hz}, 1 \mathrm{H}), 4.09-3.98(\mathrm{~m}, 2 \mathrm{H}), 2.45(\mathrm{~d}, J=8.0 \mathrm{~Hz}$, $1 \mathrm{H}$ ), 2.15 (hept, $J=7.5 \mathrm{~Hz}, 2 \mathrm{H}$ ), 1.86 (ddd, $J=13.4,9.2,5.9 \mathrm{~Hz}, 1 \mathrm{H}), 1.69$ (s, 3H), 1.63 (s, 3H), 1.36 (ddd, $J=13.5,9.7,6.8 \mathrm{~Hz}, 1 \mathrm{H}), 1.12(\mathrm{~s}, 3 \mathrm{H})$.

${ }^{13}$ C NMR (Major) $\left(126 \mathrm{MHz}, \mathrm{CDCl}_{3}\right) \delta 200.3,138.9,134.5,133.1,132.4,128.7,128.3,123.8$, 123.7, 59.4, 42.9, 35.2, 35.1, 32.5, 25.9, 25.3, 17.9, 12.6 .

IR(neat) 3444, 2923, 1725, 1351, 1282, 1261, 1125, 1053, 964, 910, 759, $698 \mathrm{~cm}^{-1}$

LRMS m/z (ESI APCI) calculated for $\mathrm{C}_{17} \mathrm{H}_{16} \mathrm{O}_{2}[\mathrm{M}+\mathrm{H}]$ 273.2, found 273.2.

3ag (2-(1-hydroxyallyl)cyclopropyl)(phenyl)methanone

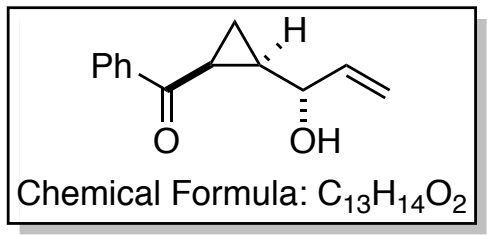

$\mathrm{Y}=73 \%$. Yellow oil. $\mathrm{R}_{\mathrm{f}}=0.22$ (4:1 Hexanes:EtOAc).

${ }^{1}$ H NMR (Major) (500 MHz, Chloroform- $d$ ) $\delta 8.03(\mathrm{~d}, J=7.7 \mathrm{~Hz}, 2 \mathrm{H}), 7.56(\mathrm{t}, J=7.4 \mathrm{~Hz}, 1 \mathrm{H})$, 7.47 (t, $J=7.6 \mathrm{~Hz}, 2 \mathrm{H}), 6.00$ (ddd, $J=16.6,10.4,5.6 \mathrm{~Hz}, 1 \mathrm{H}), 5.30$ (d, $J=17.2 \mathrm{~Hz}, 1 \mathrm{H}), 5.12$ $(\mathrm{d}, J=10.4 \mathrm{~Hz}, 1 \mathrm{H}), 4.06(\mathrm{dd}, J=9.0,5.6 \mathrm{~Hz}, 1 \mathrm{H}), 2.81(\mathrm{td}, J=8.1,5.7 \mathrm{~Hz}, 1 \mathrm{H}), 2.16(\mathrm{~s}, 1 \mathrm{H})$, $1.75(\mathrm{p}, J=8.4 \mathrm{~Hz}, 1 \mathrm{H}), 1.49$ (q, $J=5.8 \mathrm{~Hz}, 1 \mathrm{H}), 1.26(\mathrm{td}, J=7.9,4.5 \mathrm{~Hz}, 2 \mathrm{H})$.

${ }^{13}$ C NMR (Major) $\left(126 \mathrm{MHz}, \mathrm{CDCl}_{3}\right) \delta 199.9,139.9,138.6,133.1,128.7,128.4,114.9,71.0$, 31.1, 23.1, 13.0.

IR(neat) 3407, 2889, 1666, 1391, 1224, 1003, 714, $690 \mathrm{~cm}^{-1}$

LRMS m/z (ESI APCI) calculated for $\mathrm{C}_{13} \mathrm{H}_{14} \mathrm{O}_{2}[\mathrm{M}+\mathrm{H}]$ 203.1, found 203.1. 
3ah (2-(1-hydroxyethyl)cyclopropyl)(phenyl)methanone

Chemical Formula: $\mathrm{C}_{12} \mathrm{H}_{14} \mathrm{O}_{2}$

$\mathrm{Y}=69 \%$. Off-white solid. $\mathrm{R}_{\mathrm{f}}=0.26$ (4:1 Hexanes:EtOAc).

${ }^{1}$ H NMR (Major) $(500 \mathrm{MHz}$, Chloroform- $d$ ) $\delta 8.08-8.02(\mathrm{~m}, 2 \mathrm{H}), 7.60-7.53(\mathrm{~m}, 1 \mathrm{H}), 7.47(\mathrm{t}$, $J=7.6 \mathrm{~Hz}, 2 \mathrm{H}), 3.74(\mathrm{dt}, J=12.6,6.4 \mathrm{~Hz}, 1 \mathrm{H}), 2.76(\mathrm{td}, J=8.2,5.7 \mathrm{~Hz}, 1 \mathrm{H}), 1.95(\mathrm{~s}, 1 \mathrm{H}), 1.69$ (qd, $J=8.7,6.9 \mathrm{~Hz}, 1 \mathrm{H}), 1.43-1.35(\mathrm{~m}, 1 \mathrm{H}), 1.33(\mathrm{~d}, J=6.3 \mathrm{~Hz}, 3 \mathrm{H}), 1.25(\mathrm{td}, J=8.2,4.4 \mathrm{~Hz}$, $1 \mathrm{H})$.

${ }^{13}$ C NMR (Major) (126 MHz, $\left.\mathrm{CDCl}_{3}\right) \delta$ 199.9, 138.5, 133.1, 128.7, 128.4, 66.6, 33.0, 23.4, 23.2, 13.2.

IR(neat) 3497, 2965, 1666, 1599, 1390, 1210, 999, 699, $690 \mathrm{~cm}^{-1}$

LRMS m/z (ESI APCI) calculated for $\mathrm{C}_{12} \mathrm{H}_{14} \mathrm{O}_{2}[\mathrm{M}+\mathrm{H}]$ 191.1, found 191.1.

3ai (2-(hydroxy(phenyl)methyl)cyclopropyl)(phenyl)methanone

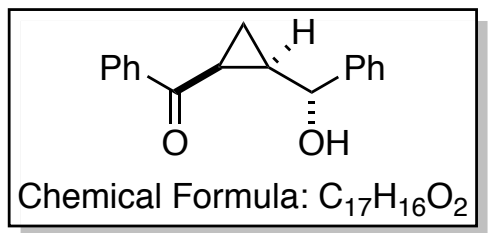

$\mathrm{Y}=62 \%$. Light yellow solid. $\mathrm{R}_{\mathrm{f}}=0.54$ (4:1 Hexanes:EtOAc).

${ }^{1}$ H NMR (500 MHz, Chloroform- $d$ ) $\delta 8.17-8.06(\mathrm{~m}, 2 \mathrm{H}), 7.61(\mathrm{t}, J=7.4 \mathrm{~Hz}, 1 \mathrm{H}), 7.56-7.47$ $(\mathrm{m}, 4 \mathrm{H}), 7.39(\mathrm{t}, J=7.5 \mathrm{~Hz}, 2 \mathrm{H}), 7.32(\mathrm{t}, J=7.3 \mathrm{~Hz}, 1 \mathrm{H}), 4.64(\mathrm{~d}, J=9.4 \mathrm{~Hz}, 1 \mathrm{H}), 2.93(\mathrm{td}, J=$ $8.1,5.7 \mathrm{~Hz}, 1 \mathrm{H}), 2.26(\mathrm{~s}, 1 \mathrm{H}), 2.01(\mathrm{qd}, J=8.7,6.9 \mathrm{~Hz}, 1 \mathrm{H}), 1.64(\mathrm{dt}, J=6.8,5.0 \mathrm{~Hz}, 1 \mathrm{H}), 1.26$ (dt, $J=8.2,4.1 \mathrm{~Hz}, 1 \mathrm{H})$.

${ }^{13}$ C NMR $\left(126 \mathrm{MHz}, \mathrm{CDCl}_{3}\right) \delta 199.6,144.0,138.6,133.0,128.7,128.6,128.4,127.7,126.0$, 72.1, 33.0, 23.8, 13.4 .

IR(neat) 3495, 2944, 1669, 1591, 1390, 1223, 1210, 1010, $699 \mathrm{~cm}^{-1}$

LRMS m/z (ESI APCI) calculated for $\mathrm{C}_{17} \mathrm{H}_{16} \mathrm{O}_{2}[\mathrm{M}+\mathrm{H}]$ 253.1, found 253.1. 
3aj (2-(1-hydroxyethyl)-3-methylcyclopropyl)(phenyl)methanone

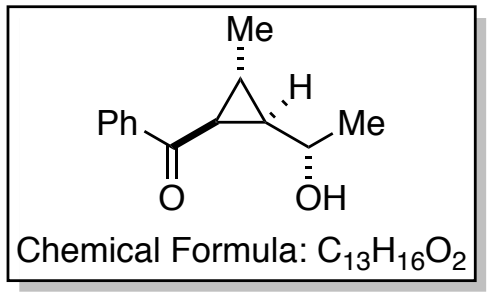

$\mathrm{Y}=88 \%$ Pale-yellow oil. $\mathrm{R}_{\mathrm{f}}=0.19$ (4:1 Hexanes:EtOAc)

${ }^{1}$ H NMR $(500 \mathrm{MHz}$, Chloroform- $d$ ) $\delta 8.05-7.98(\mathrm{~m}, 2 \mathrm{H}), 7.60-7.53(\mathrm{~m}, 1 \mathrm{H}), 7.51-7.43(\mathrm{~m}$, $2 \mathrm{H}), 3.87$ (dq, $J=8.8,6.3 \mathrm{~Hz}, 1 \mathrm{H}), 2.47$ (dd, $J=8.4,5.1 \mathrm{~Hz}, 1 \mathrm{H}), 2.23(\mathrm{~s}, 1 \mathrm{H}), 1.74$ (td, $J=6.3$, $5.2 \mathrm{~Hz}, 1 \mathrm{H}), 1.50$ (td, $J=8.6,6.4 \mathrm{~Hz}, 1 \mathrm{H}), 1.32(\mathrm{~d}, J=6.3 \mathrm{~Hz}, 3 \mathrm{H}), 1.24(\mathrm{~d}, J=6.1 \mathrm{~Hz}, 3 \mathrm{H})$.

${ }^{13} \mathbf{C}$ NMR $\left(126 \mathrm{MHz}, \mathrm{CDCl}_{3}\right) \delta 200.2,138.6,133.0,128.7,128.7,128.7,128.2,66.0,42.2,32.0$, 23.2, 23.1, 18.2.

IR(neat) $3214,2975,1735,1602,1224,1115,1062,905,732,715 \mathrm{~cm}^{-1}$

LRMS m/z (ESI APCI) calculated $\mathrm{C}_{13} \mathrm{H}_{16} \mathrm{O}_{2}[\mathrm{M}+\mathrm{H}]$ 205.1, found 205.1 .

3ak (2-(hydroxy(phenyl)methyl)-3-propylcyclopropyl)(phenyl)methanone

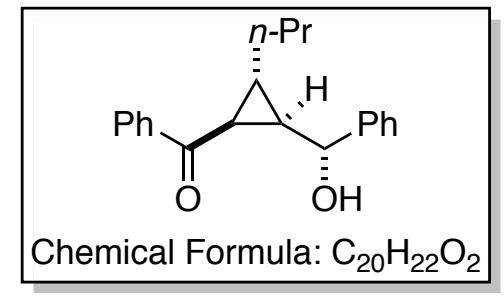

$\mathrm{Y}=75 \%$. Off-white solid. $\mathrm{R}_{\mathrm{f}}=0.34$ (4:1 Hexanes:EtOAc).

${ }^{1}$ H NMR (500 MHz, Chloroform- $d$ ) $\delta 8.09-8.02(\mathrm{~m}, 2 \mathrm{H}), 7.61-7.54(\mathrm{~m}, 1 \mathrm{H}), 7.52-7.42(\mathrm{~m}$, $4 \mathrm{H}), 7.36(\mathrm{t}, J=7.6 \mathrm{~Hz}, 2 \mathrm{H}), 7.31-7.24(\mathrm{~m}, 1 \mathrm{H}), 4.77(\mathrm{dd}, J=9.2,3.7 \mathrm{~Hz}, 1 \mathrm{H}), 2.66(\mathrm{dd}, J=$ 8.4, $5.1 \mathrm{~Hz}, 1 \mathrm{H}), 2.28$ (d, $J=3.8 \mathrm{~Hz}, 1 \mathrm{H}), 1.99(\mathrm{qd}, J=6.6,5.0 \mathrm{~Hz}, 1 \mathrm{H}), 1.81(\mathrm{td}, J=8.9,6.5$ $\mathrm{Hz}, 1 \mathrm{H}), 1.48-1.37(\mathrm{~m}, 1 \mathrm{H}), 1.31-1.26(\mathrm{~m}, 1 \mathrm{H}), 1.22$ (ddtd, $J=12.6,8.1,6.7,6.3,4.4 \mathrm{~Hz}$, $2 \mathrm{H}), 0.77(\mathrm{t}, J=7.2 \mathrm{~Hz}, 3 \mathrm{H})$.

${ }^{13} \mathbf{C}$ NMR $\left(126 \mathrm{MHz}, \mathrm{CDCl}_{3}\right) \delta 199.8,144.1,138.7,132.9,128.7,128.6,127.6,126.0,71.6$, 41.3, 35.2, 31.2, 28.6, 22.1, 13.8 .

IR(neat) 3437, 2958, 2923, 1666, 1587, 1442, 1251, 1032, 908, $730 \mathrm{~cm}^{-1}$

LRMS m/z (ESI APCI) calculated for $\mathrm{C}_{20} \mathrm{H}_{22} \mathrm{O}_{2}[\mathrm{M}+\mathrm{H}]$ 295.2, found 295.2. 
3al (2-(cyclohexyl(hydroxy)methyl)-3-propylcyclopropyl)(phenyl)methanone

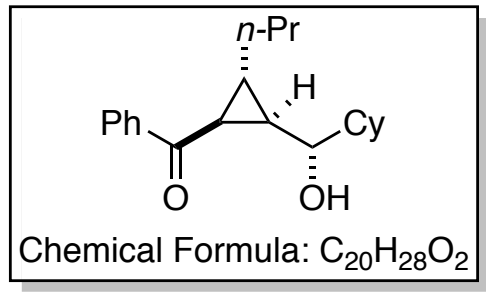

$\mathrm{Y}=95 \%$. Off-white solid. $\mathrm{R}_{\mathrm{f}}=0.54$ (4:1 Hexanes:EtOAc).

${ }^{1}$ H NMR $(500 \mathrm{MHz}$, Chloroform- $d$ ) $\delta 8.06-7.97(\mathrm{~m}, 2 \mathrm{H}), 7.54(\mathrm{t}, J=7.5 \mathrm{~Hz}, 1 \mathrm{H}), 7.45(\mathrm{t}, J=$ $7.6 \mathrm{~Hz}, 2 \mathrm{H}), 3.41$ (dd, $J=9.2,6.6 \mathrm{~Hz}, 1 \mathrm{H}), 2.49$ (dd, $J=8.7,5.0 \mathrm{~Hz}, 1 \mathrm{H}), 1.99-1.86(\mathrm{~m}, 2 \mathrm{H})$, $1.86-1.80(\mathrm{~m}, 1 \mathrm{H}), 1.80-1.71(\mathrm{~m}, 3 \mathrm{H}), 1.62(\mathrm{dtq}, J=12.8,10.2,3.8,2.9 \mathrm{~Hz}, 3 \mathrm{H}), 1.43(\mathrm{dt}, J=$ $14.8,7.4 \mathrm{~Hz}, 3 \mathrm{H}), 1.32-1.15(\mathrm{~m}, 4 \mathrm{H}), 1.15-1.00(\mathrm{~m}, 2 \mathrm{H}), 0.92(\mathrm{t}, J=7.3 \mathrm{~Hz}, 3 \mathrm{H})$.

${ }^{13} \mathbf{C}$ NMR $\left(126 \mathrm{MHz}, \mathrm{CDCl}_{3}\right) \delta 200.3,138.8,132.8,128.6,128.3,73.7,44.4,38.8,35.4,29.3$, 29.0, 26.7, 26.4, 26.3, 22.2, 14.1 .

IR(neat) 3438, 2922, 2851, 1656, 1451, 1232, 1020, 711, 685, $660 \mathrm{~cm}^{-1}$

LRMS m/z (ESI APCI) calculated for $\mathrm{C}_{20} \mathrm{H}_{28} \mathrm{O}_{2}[\mathrm{M}+\mathrm{H}]$ 301.2, found 301.2.

3an (2-hydroxybicyclo[6.1.0]nonan-9-yl)(phenyl)methanone

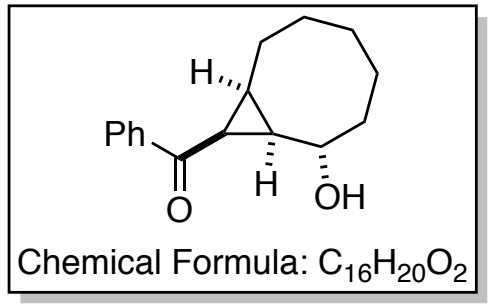

$\mathrm{Y}=85 \%$. Colorless oil. $\mathrm{R}_{\mathrm{f}}=0.05$ (4:1 Hexanes:EtOAc)

${ }^{1}$ H NMR $(500 \mathrm{MHz}$, Chloroform- $d$ ) $\delta 8.10-7.98(\mathrm{~m}, 2 \mathrm{H}), 7.57(\mathrm{t}, J=7.4 \mathrm{~Hz}, 1 \mathrm{H}), 7.47(\mathrm{t}, J=$ $7.6 \mathrm{~Hz}, 2 \mathrm{H}), 4.13(\mathrm{td}, J=10.9,4.6 \mathrm{~Hz}, 1 \mathrm{H}), 3.14(\mathrm{~s}, 1 \mathrm{H}), 2.58(\mathrm{dd}, J=9.6,7.7 \mathrm{~Hz}, 1 \mathrm{H}), 1.91$ (tt, $J=12.0,4.3 \mathrm{~Hz}, 1 \mathrm{H}), 1.77$ (tdd, $J=17.0,8.4,4.2 \mathrm{~Hz}, 3 \mathrm{H}), 1.73-1.65$ (m, 2H), 1.59 (ddt, $J=$ $15.3,12.2,4.3 \mathrm{~Hz}, 1 \mathrm{H}), 1.49$ (tdd, $J=13.6,10.4,4.4 \mathrm{~Hz}, 1 \mathrm{H}), 1.45-1.30(\mathrm{~m}, 2 \mathrm{H}), 1.21$ (tdd, $J=$ $14.3,8.0,3.6 \mathrm{~Hz}, 2 \mathrm{H})$.

${ }^{13}$ C NMR $\left(126 \mathrm{MHz}, \mathrm{CDCl}_{3}\right) \delta 200.3,138.4,133.4,128.7,128.6,67.7,36.7,30.4,29.8,27.0$, 26.6, 26.2, 25.1, 24.5.

IR(neat) 3446, 2924, 2854, 1660, 1448, 1394, 1212, 1050, 1011, 956, $718 \mathrm{~cm}^{-1}$

LRMS m/z (ESI APCI) calculated for $\mathrm{C}_{16} \mathrm{H}_{20} \mathrm{O}_{2}[\mathrm{M}+\mathrm{H}]$ 245.3, found 245.3. 
Deuterated allylic alcohol-Retention of stereochemistry at the alkene<smiles></smiles>

1a<smiles>[2H]/C(=C\CO)CO</smiles>

2a- $d_{1}$
$\stackrel{\left[\mathrm{Cp}{ }^{* \mathrm{CF} 3} \mathrm{RhCl}_{2}\right]_{2}(5 \mathrm{~mol} \%)}{\longrightarrow}$

KOPiv ( 2 equiv.)

TFE, $0{ }^{\circ} \mathrm{C}$

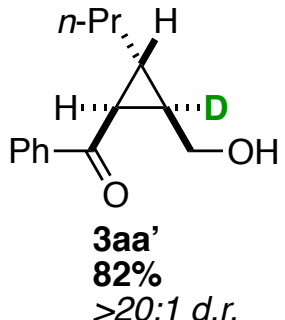

Figure 15.

$N$-enoxyphthalimide 1a $(0.12 \mathrm{mmol})$, catalyst $\left[\mathrm{Cp}^{*}{ }^{\mathrm{CF} 3} \mathrm{RhCl}_{2}\right]_{2}(5 \mathrm{~mol} \%, 0.006 \mathrm{mmol}, 4.4 \mathrm{mg})$, and KOPiv ( 2 equiv., $0.24 \mathrm{mmol}, 33.6 \mathrm{mg}$ ) were weighed in a 1-dram vial with a magnetic stirbar. Cooled TFE $(0.2 \mathrm{M}, 600 \mu \mathrm{L})$ was added followed by allylic alcohol $\mathbf{2 a - d _ { 1 }}$ (1.2 equiv., $0.144 \mathrm{mmol})$. The vial was sealed with a screw-cap and placed in an aluminium block cooled to $0{ }^{\circ} \mathrm{C}$ surrounded by ice in an insulated box and stirred for 16 hours. Upon completion judged by TLC, TFE was removed by rotary evaporation and the residue was taken up in EtOAc and filtered through a silica plug flushing with EtOAc. The filtrate was concentrated to $\sim 1 \mathrm{~mL}$ and transferred to a 1.5-dram vial where the solution was partitioned with the addition of $10 \% \mathrm{NaOH}$ solution. The aqueous layer was extracted three times with EtOAc and the combined organic extracts were filtered through a pad of celite ${ }^{\circledR}$ and $\mathrm{Na}_{2} \mathrm{SO}_{4}$ then concentrated. The residue was purified by flash chromatography (Hexane:EtOAc, 19:1 $\rightarrow 9: 1 \rightarrow 4: 1$ ) to afford the cyclopropane product.

3aa' 2-(hydroxymethyl)-3-propylcyclopropyl-2-d)(phenyl)methanone

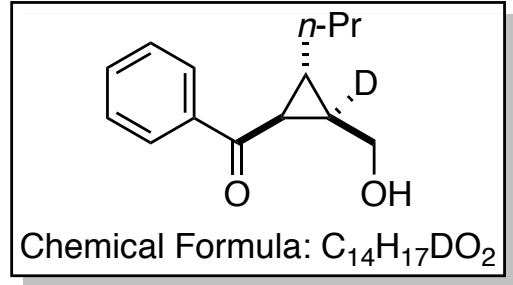

$\mathrm{Y}=82 \%$. Colorless Oil. $\mathrm{R}_{\mathrm{f}}=0.22$ (4:1 Hexanes:EtOAc).

${ }^{1}$ H NMR $(500 \mathrm{MHz}$, Chloroform- $d$ ) $\delta 8.05-7.97(\mathrm{~m}, 2 \mathrm{H}), 7.62-7.55(\mathrm{~m}, 1 \mathrm{H}), 7.49$ (dd, $J=$ 8.4, 7.0 Hz, 2H), $3.96(\mathrm{~d}, J=12.0 \mathrm{~Hz}, 1 \mathrm{H}), 3.77(\mathrm{~d}, J=12.0 \mathrm{~Hz}, 1 \mathrm{H}), 2.56(\mathrm{~d}, J=5.0 \mathrm{~Hz}, 1 \mathrm{H})$, $2.21(\mathrm{~s}, 1 \mathrm{H}), 1.86(\mathrm{q}, J=6.2 \mathrm{~Hz}, 1 \mathrm{H}), 1.48(\mathrm{ttd}, J=11.8,5.8,5.1,2.5 \mathrm{~Hz}, 4 \mathrm{H}), 0.99-0.92(\mathrm{~m}$, $3 \mathrm{H})$.

${ }^{13}$ C NMR $\left(126 \mathrm{MHz}, \mathrm{CDCl}_{3}\right) \delta 199.6,144.0,138.6,133.0,128.7,128.6,128.4,127.7,126.0$, 72.1, 33.0, 23.8, 13.4 .

IR(neat) $3458,2921,1657,1450,1228,1020,699 \mathrm{~cm}^{-1}$

LRMS m/z (ESI APCI) calculated for $\mathrm{C}_{17} \mathrm{H}_{16} \mathrm{O}_{2}[\mathrm{M}+\mathrm{H}] 220.1$, found 220.1. 


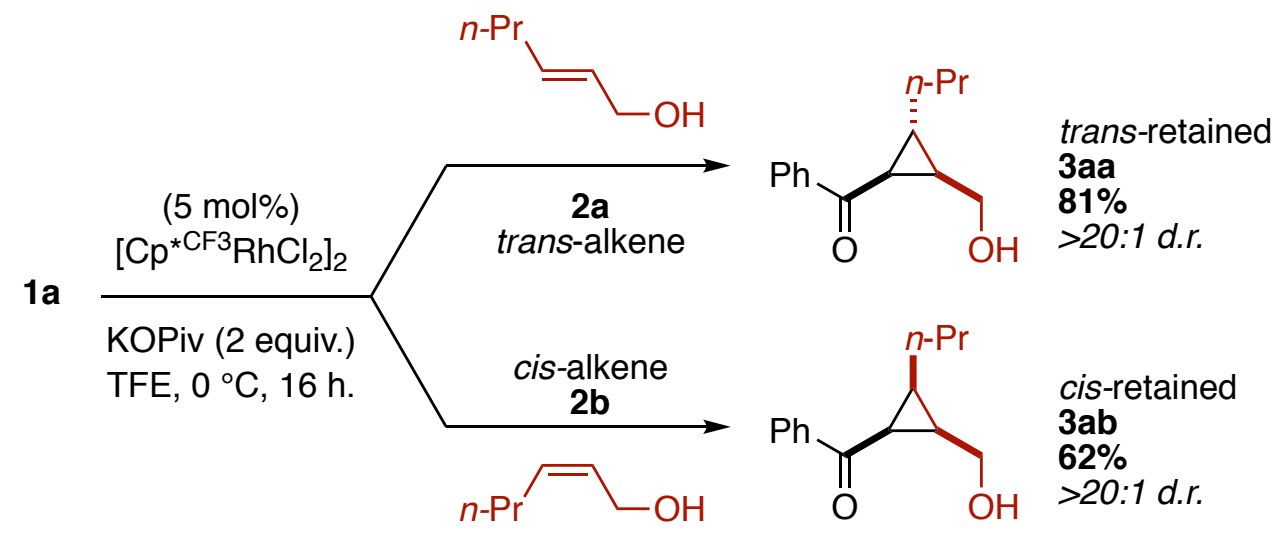

Figure 16.

We assume retention of stereochemistry at the double bond based on results achieved with allylic alcohols $\mathbf{2 a}$ and $\mathbf{2 b}$. For each of these reactions, the stereochemistry of the double bond is shown in the product-trans-alkenes give trans-cyclopropanes and cis-alkenes give cis-cyclopropanes.
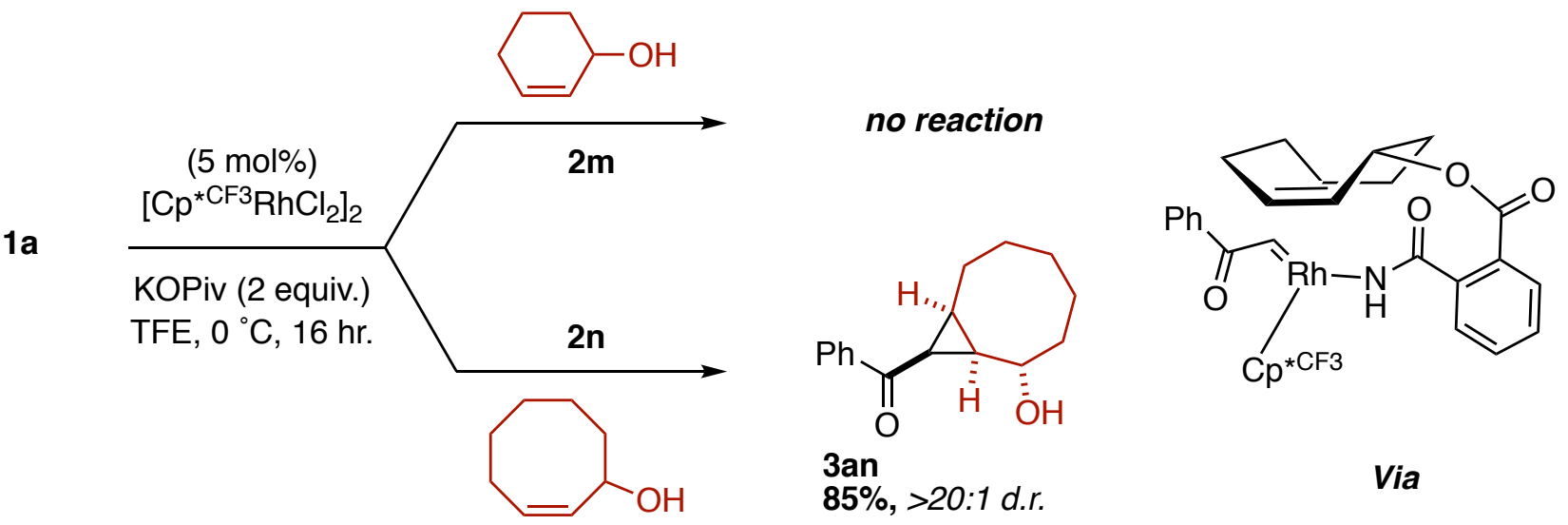

Figure 17. 


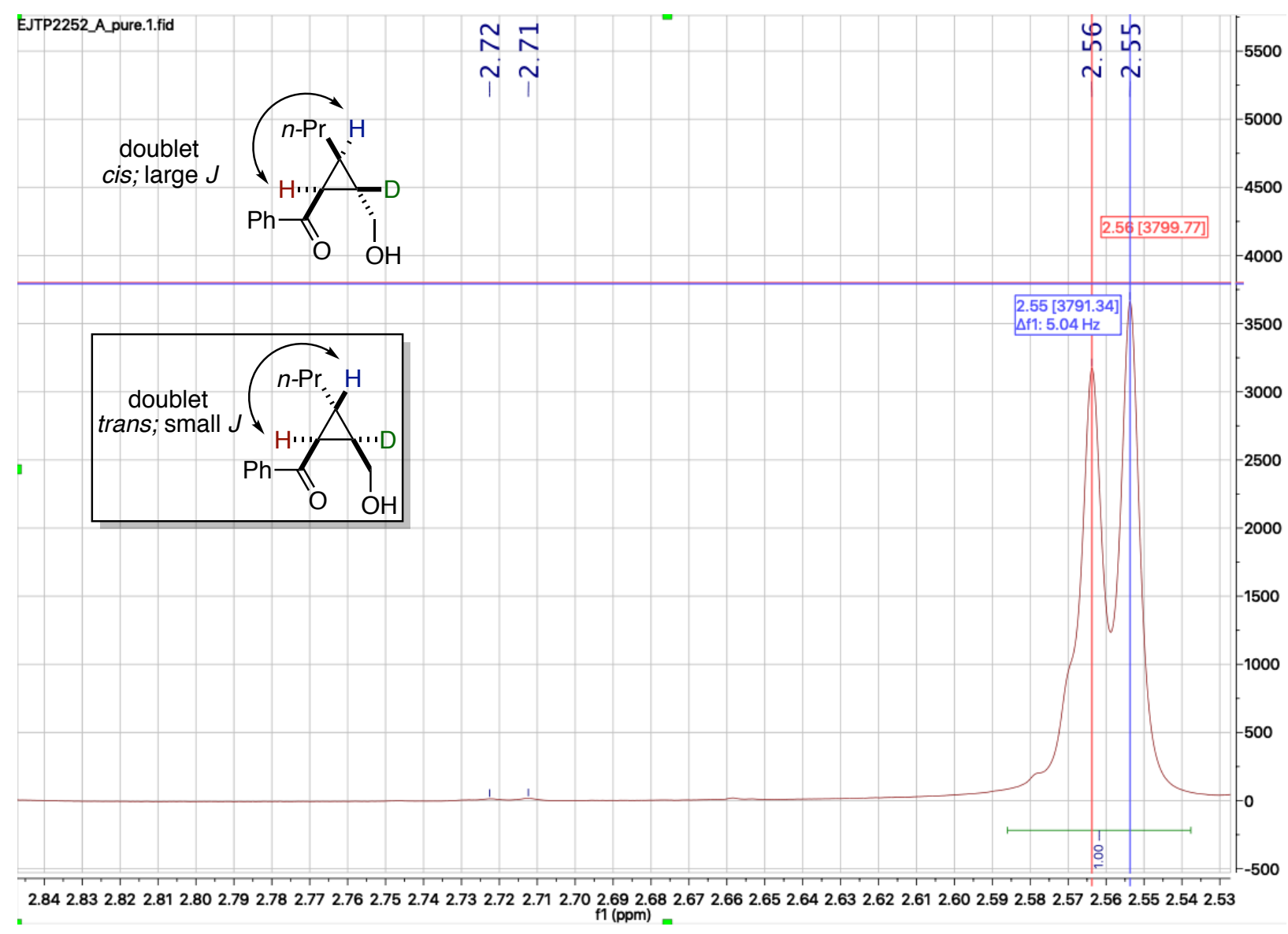

Figure 18.

Furthermore, when $\mathbf{2 a -} \boldsymbol{d}_{\boldsymbol{1}}$ is subjected to the reaction conditions, the resulting cyclopropane 3aa' is characterized by coupling constants of the $\alpha$-hydrogen to the phenyl ketone (highlighted in red) that gives a doublet in the ${ }^{1} \mathrm{H}-\mathrm{NMR}$ spectrum. Assuming retention of stereochemistry at the alkene, if the hydroxymethyl substituent is trans to the ketone, the blue proton from the alkene should be cis to the ketone and give a large $J$ value. Alternatively, if the hydroxymethyl substituent is cis to the ketone, the blue proton from the alkene should be trans to the ketone and give a small $J$ value. We observe the doublet of the $\alpha$-hydrogen to have a $J$ value of $5.0 \mathrm{~Hz}$ indicating formation of the diastereomer in the highlighted box. ${ }^{12}$ 


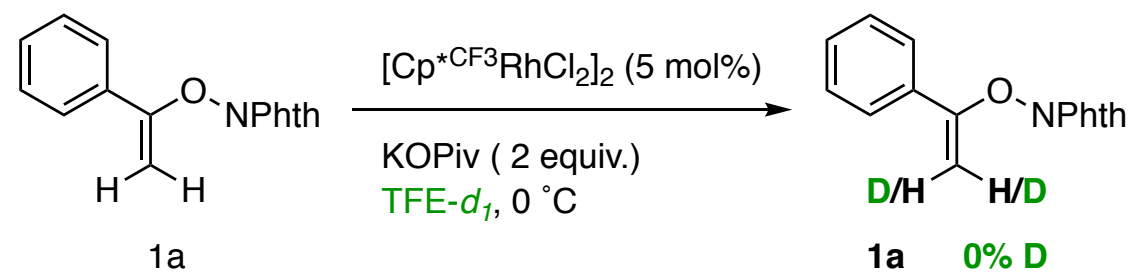

Figure 19.

$N$-enoxyphthalimide 1a $(0.12 \mathrm{mmol})$, catalyst $\left[\mathrm{Cp}^{*}{ }^{\mathrm{CF} 3} \mathrm{RhCl}_{2}\right]_{2}(5 \mathrm{~mol} \%, 0.006 \mathrm{mmol}, 4.4 \mathrm{mg})$, and KOPiv (2 equiv., $0.24 \mathrm{mmol}, 33.6 \mathrm{mg}$ ) were weighed in a 1-dram vial with a magnetic stirbar. Cooled TFE $(0.2 \mathrm{M}, 600 \mu \mathrm{L})$ was added. The vial was sealed with a screw-cap and placed in an aluminium block cooled to $0{ }^{\circ} \mathrm{C}$ surrounded by ice in an insulated box and stirred for 3 hours. TFE- $d_{l}$ was removed by rotary evaporation and the residue was taken up in EtOAc and filtered through a silica plug flushing with EtOAc. The filtrate was concentrated to $\sim 1 \mathrm{~mL}$ and transferred to a 1.5-dram vial where the solution was partitioned with the addition of $10 \% \mathrm{NaOH}$ solution. The aqueous layer was extracted three times with EtOAc and the combined organic extracts were filtered through a pad of celite ${ }^{\circledR}$ and $\mathrm{Na}_{2} \mathrm{SO}_{4}$ then concentrated. The residue was purified by flash chromatography (Hexane:EtOAc, 19:1 $\rightarrow 9: 1 \rightarrow 4: 1$ ) to re-isolate the starting material.

\section{With homoallylic alcohol $\mathbf{4 a}$}

$1 a$<smiles>OCC/C=C/P</smiles>

$4 a$
$\left[\mathrm{Cp}{ }^{* \mathrm{CF} 3} \mathrm{RhCl}_{2}\right]_{2}(5 \mathrm{~mol} \%)$ KOPiv (2 equiv.) TFE, $0{ }^{\circ} \mathrm{C}$

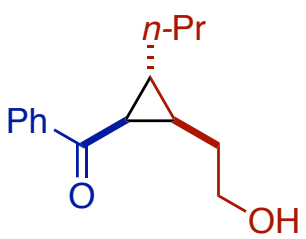

5aa $12 \%,>20: 1$ d.r.

Figure 20.

$N$-enoxyphthalimide 1a $(0.12 \mathrm{mmol})$, catalyst $\left[\mathrm{Cp}^{*}{ }^{\mathrm{CF} 3} \mathrm{RhCl}_{2}\right]_{2}(5 \mathrm{~mol} \%, 0.006 \mathrm{mmol}, 4.4 \mathrm{mg})$, and KOPiv (2 equiv., $0.24 \mathrm{mmol}, 33.6 \mathrm{mg}$ ) were weighed in a 1-dram vial with a magnetic stirbar. Cooled TFE $(0.2 \mathrm{M}, 600 \mu \mathrm{L})$ was added followed by homoallylic alcohol 4a (1.2 equiv., $0.144 \mathrm{mmol})$. The vial was sealed with a screw-cap and placed in an aluminium block cooled to $0{ }^{\circ} \mathrm{C}$ surrounded by ice in an insulated box and stirred for 16 hours. Upon completion judged by TLC, TFE was removed by rotary evaporation and the residue was taken up in EtOAc and filtered through a silica plug flushing with EtOAc. The filtrate was concentrated to $\sim 1 \mathrm{~mL}$ and transferred to a 1.5-dram vial where the solution was partitioned with the addition of $10 \% \mathrm{NaOH}$ solution. The aqueous layer was extracted three times with EtOAc and the combined organic extracts were filtered through a pad of celite ${ }^{\circledR}$ and $\mathrm{Na}_{2} \mathrm{SO}_{4}$ then concentrated. Yield and diastereoselectivity were determined by crude ${ }^{1} \mathrm{H}$ NMR. 
With bis-homoallylic alcohol

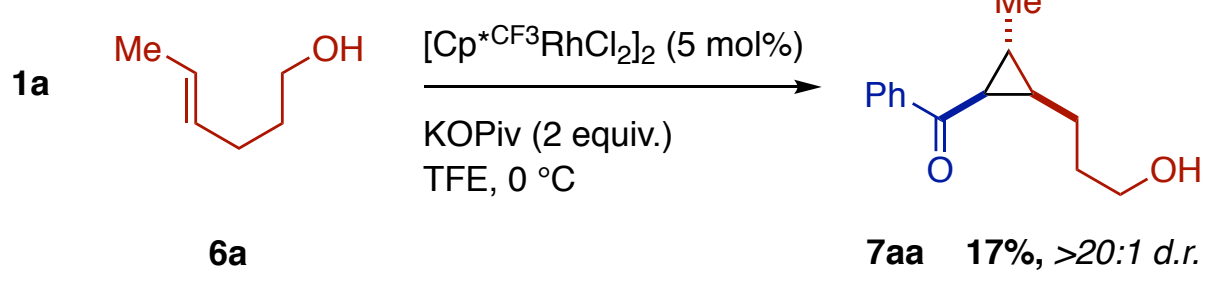

Figure 21.

$N$-enoxyphthalimide 1a $(0.12 \mathrm{mmol})$, catalyst $\left[\mathrm{Cp}^{*}{ }^{\mathrm{CF} 3} \mathrm{RhCl}_{2}\right]_{2}(5 \mathrm{~mol} \%, 0.006 \mathrm{mmol}, 4.4 \mathrm{mg})$, and KOPiv (2 equiv., $0.24 \mathrm{mmol}, 33.6 \mathrm{mg}$ ) were weighed in a 1-dram vial with a magnetic stirbar. Cooled TFE $(0.2 \mathrm{M}, 600 \mu \mathrm{L})$ was added followed by bis-homoallylic alcohol 6a (1.2 equiv., $0.144 \mathrm{mmol}$ ). The vial was sealed with a screw-cap and placed in an aluminium block cooled to $0{ }^{\circ} \mathrm{C}$ surrounded by ice in an insulated box and stirred for 16 hours. Upon completion judged by TLC, TFE was removed by rotary evaporation and the residue was taken up in EtOAc and filtered through a silica plug flushing with EtOAc. The filtrate was concentrated to $\sim 1 \mathrm{~mL}$ and transferred to a 1.5-dram vial where the solution was partitioned with the addition of $10 \%$ $\mathrm{NaOH}$ solution. The aqueous layer was extracted three times with EtOAc and the combined organic extracts were filtered through a pad of celite ${ }^{\circledR}$ and $\mathrm{Na}_{2} \mathrm{SO}_{4}$ then concentrated. Yield and diastereoselectivity were determined by crude ${ }^{1} \mathrm{H}$ NMR.

With allylic ether $\mathbf{6 a}$

$1 a$

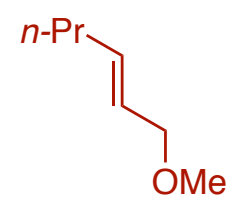

$8 \mathbf{a}$

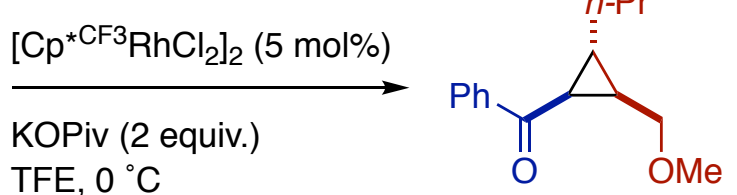

9aa

Figure 22.

$N$-enoxyphthalimide 1a $(0.12 \mathrm{mmol})$, catalyst $\left[\mathrm{Cp}^{*}{ }^{\mathrm{CF} 3} \mathrm{RhCl}_{2}\right]_{2}(5 \mathrm{~mol} \%, 0.006 \mathrm{mmol}, 4.4 \mathrm{mg})$, and KOPiv (2 equiv., $0.24 \mathrm{mmol}, 33.6 \mathrm{mg}$ ) were weighed in a 1-dram vial with a magnetic stirbar. Cooled TFE $(0.2 \mathrm{M}, 600 \mu \mathrm{L})$ was added followed by allylic ether 8a (1.2 equiv., 0.144 $\mathrm{mmol}$ ). The vial was sealed with a screw-cap and placed in an aluminium block cooled to $0{ }^{\circ} \mathrm{C}$ surrounded by ice in an insulated box and stirred for 16 hours. Upon completion judged by TLC, TFE was removed by rotary evaporation and the residue was taken up in EtOAc and filtered through a silica plug flushing with EtOAc. The filtrate was concentrated to $\sim 1 \mathrm{~mL}$ and transferred to a 1.5-dram vial where the solution was partitioned with the addition of $10 \% \mathrm{NaOH}$ solution. The aqueous layer was extracted three times with EtOAc and the combined organic extracts were filtered through a pad of celite ${ }^{\circledR}$ and $\mathrm{Na}_{2} \mathrm{SO}_{4}$ then concentrated. Yield was determined by crude ${ }^{1} \mathrm{H}$ NMR. 
With allylic carboxylic acid

$1 \mathrm{a}$

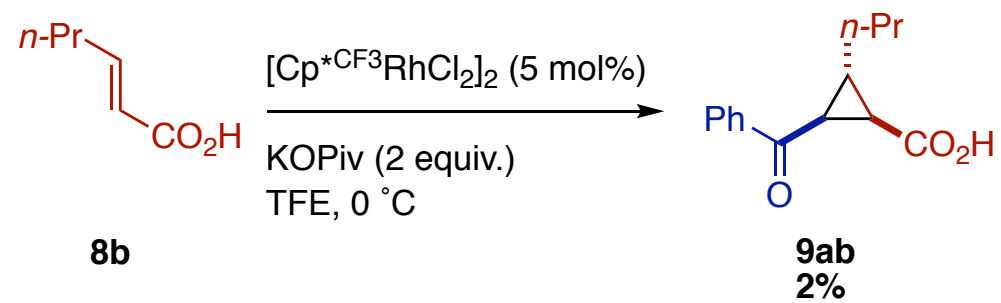

Figure 23.

$N$-enoxyphthalimide $(0.12 \mathrm{mmol})$, catalyst $\left[\mathrm{Cp}^{*}{ }^{\mathrm{CF} 3} \mathrm{RhCl}_{2}\right]_{2}(5 \mathrm{~mol} \%, 0.006 \mathrm{mmol}, 4.4 \mathrm{mg})$, and KOPiv (2 equiv., $0.24 \mathrm{mmol}, 33.6 \mathrm{mg}$ ) were weighed in a 1-dram vial with a magnetic stirbar. Cooled TFE $(0.2 \mathrm{M}, 600 \mu \mathrm{L})$ was added followed by allylic carboxylic acid (1.2 equiv., 0.144 mmol). The vial was sealed with a screw-cap and placed in an aluminium block cooled to $0{ }^{\circ} \mathrm{C}$ surrounded by ice in an insulated box and stirred for 16 hours. Upon completion judged by TLC, TFE was removed by rotary evaporation and the residue was taken up in EtOAc and filtered through a silica plug flushing with EtOAc. The filtrate was concentrated to $\sim 1 \mathrm{~mL}$ and transferred to a 1.5-dram vial where the solution was partitioned with the addition of $10 \% \mathrm{NaOH}$ solution. The aqueous layer was extracted three times with EtOAc and the combined organic extracts were filtered through a pad of celite ${ }^{\circledR}$ and $\mathrm{Na}_{2} \mathrm{SO}_{4}$ then concentrated. Yield awas determined by crude ${ }^{1} \mathrm{H}$ NMR.

With allylic amine

$1 \mathrm{a}$

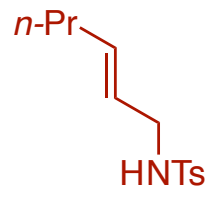

$8 \mathrm{c}$
$\left[\mathrm{Cp}{ }^{* \mathrm{CF} 3} \mathrm{RhCl}_{2}\right]_{2}(5 \mathrm{~mol} \%)$

KOPiv ( 2 equiv.)

$\mathrm{TFE}, 0{ }^{\circ} \mathrm{C}$

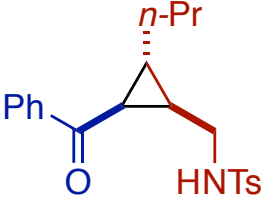

9ac

77\%, 9.5:1 d.r.

Figure 24.

$\mathrm{N}$-enoxyphthalimide $(0.12 \mathrm{mmol})$, catalyst $\left[\mathrm{Cp}^{*}{ }^{\mathrm{CF} 3} \mathrm{RhCl}_{2}\right]_{2}(5 \mathrm{~mol} \%, 0.006 \mathrm{mmol}, 4.4 \mathrm{mg})$, and KOPiv (2 equiv., $0.24 \mathrm{mmol}, 33.6 \mathrm{mg}$ ) were weighed in a 1-dram vial with a magnetic stirbar. Cooled TFE $(0.2 \mathrm{M}, 600 \mu \mathrm{L})$ was added followed by allylic amine (1.2 equiv., $0.144 \mathrm{mmol})$. The vial was sealed with a screw-cap and placed in an aluminium block cooled to $0{ }^{\circ} \mathrm{C}$ surrounded by ice in an insulated box and stirred for 16 hours. Upon completion judged by TLC, TFE was removed by rotary evaporation and the residue was taken up in EtOAc and filtered through a silica plug flushing with EtOAc. The filtrate was concentrated to $\sim 1 \mathrm{~mL}$ and transferred to a 1.5dram vial where the solution was partitioned with the addition of $10 \% \mathrm{NaOH}$ solution. The aqueous layer was extracted three times with EtOAc and the combined organic extracts were filtered through a pad of celite ${ }^{\circledR}$ and $\mathrm{Na}_{2} \mathrm{SO}_{4}$ then concentrated. The crude residue was purified by flash chromatography (Hexane:EtOAc, 19:1 $\rightarrow 9: 1 \rightarrow 4: 1$ ) to afford the cyclopropane product. 


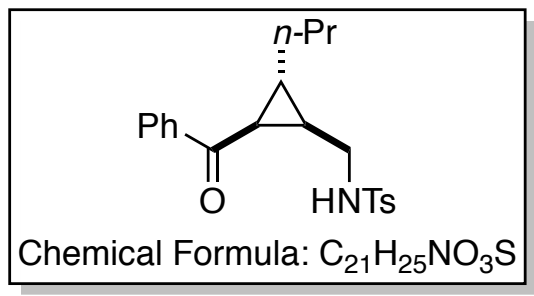

$\mathrm{Y}=77 \%$. Off-White Solid. $\mathrm{R}_{\mathrm{f}}=0.21$ (4:1 Hexanes:EtOAc).

${ }^{1}$ H NMR $(500 \mathrm{MHz}$, Chloroform- $d$ ) $\delta 7.91(\mathrm{dd}, J=8.3,1.3 \mathrm{~Hz}, 2 \mathrm{H}), 7.66(\mathrm{~d}, J=8.2 \mathrm{~Hz}, 2 \mathrm{H})$, $7.60-7.53(\mathrm{~m}, 1 \mathrm{H}), 7.46(\mathrm{t}, J=7.7 \mathrm{~Hz}, 2 \mathrm{H}), 7.25(\mathrm{~d}, J=8.0 \mathrm{~Hz}, 2 \mathrm{H}), 4.65(\mathrm{t}, J=6.4 \mathrm{~Hz}, 1 \mathrm{H})$, 3.37 (ddd, $J=14.0,6.9,5.6 \mathrm{~Hz}, 1 \mathrm{H}$ ), 3.11 (ddd, $J=14.3,8.8,5.9 \mathrm{~Hz}, 1 \mathrm{H}$ ), 2.49 (dd, $J=8.4,5.0$ $\mathrm{Hz}, 1 \mathrm{H}), 2.40(\mathrm{~s}, 3 \mathrm{H}), 1.71(\mathrm{tt}, J=8.6,6.0 \mathrm{~Hz}, 1 \mathrm{H}), 1.67-1.60(\mathrm{~m}, 1 \mathrm{H}), 1.42-1.30(\mathrm{~m}, 4 \mathrm{H})$, $0.94-0.85(\mathrm{~m}, 3 \mathrm{H})$.

${ }^{13}$ C NMR $\left(126 \mathrm{MHz}, \mathrm{CDCl}_{3}\right) \delta 199.3,143.4,134.5,133.2,129.8,128.8,128.2,127.2,123.8$, $41.3,35.1,33.0,29.8,29.7,22.3,21.7,13.9$.

IR(neat) 3520, 3189, 3061, 1666, 1602, 1373, 1305, 1239, 1159, 1049, 711, 647.

LRMS m/z (ESI APCI) calculated for $\mathrm{C}_{21} \mathrm{H}_{25} \mathrm{O}_{3} \mathrm{~S}[\mathrm{M}+\mathrm{H}]$ 372.1, found 372.1.

With $\mathrm{NaH}$<smiles>C=C(O[PH2+]c1ccccc1)c1ccccc1</smiles><smiles>OC/C=C/P</smiles>

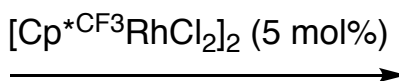

$\mathrm{NaH}$ (2 equiv.)

TFE, $0{ }^{\circ} \mathrm{C}$

Figure 25.

$N$-enoxyphthalimide $1 \mathrm{a}(0.12 \mathrm{mmol})$ and catalyst $\left[\mathrm{Cp}^{*}{ }^{\mathrm{CF} 3} \mathrm{RhCl}_{2}\right]_{2}(5 \mathrm{~mol} \%, 0.006 \mathrm{mmol}, 4.4$ $\mathrm{mg})$, were weighed in a 1-dram vial with a magnetic stirbar. Cooled TFE $(0.2 \mathrm{M}, 600 \mu \mathrm{L})$ was added followed by allylic alcohol 6a (1.2 equiv., $0.144 \mathrm{mmol})$. The vial was sealed with a screwcap and placed in an aluminium block cooled to $0{ }^{\circ} \mathrm{C}$ surrounded by ice in an insulated box and stirred for 2 mins. The vial was removed and $\mathrm{NaH}$ ( 2 equiv., $0.24 \mathrm{mmol}$ ) was added and vigorous bubbling occurred. The vial was placed back in the cooling block at $0{ }^{\circ} \mathrm{C}$ and stirred for 16 hours. Upon completion judged by TLC, TFE was removed by rotary evaporation and the residue was taken up in EtOAc and filtered through a silica plug flushing with EtOAc. The filtrate was concentrated to $\sim 1 \mathrm{~mL}$ and transferred to a 1.5-dram vial where the solution was partitioned with the addition of $10 \% \mathrm{NaOH}$ solution. The aqueous layer was extracted three times with EtOAc and the combined organic extracts were filtered through a pad of celite ${ }^{\circledR}$ and $\mathrm{Na}_{2} \mathrm{SO}_{4}$ then concentrated. Yield and diastereoselectivity were determined by crude ${ }^{1} \mathrm{H} \mathrm{NMR}$. 
<smiles>C=C(ON1C(=O)c2ccccc2C1=O)c1ccccc1</smiles>

$1 a$<smiles>CC=CCO</smiles>

2c<smiles>CC=CCOC(=O)c1ccccc1C1=NOC(C)(c2ccccc2)O1</smiles>

$10 \mathrm{ac}$

$38 \%$

Figure 26.

$N$-enoxyphthalimide 1a $(0.75 \mathrm{mmol})$ and KOPiv (1 equiv., $0.75 \mathrm{mmol})$ were weighed in a 1 dram vial with a magnetic stirbar. THF $(0.2 \mathrm{M}, 3.770 \mathrm{~mL})$ was added followed by allylic alcohol 2b (1.2 equiv., $0.91 \mathrm{mmol})$. The vial was sealed with a screw-cap and stirred for 16 hours at room temperature. THF was removed by rotary evaporation and the crude residue was purified by flash chromatography (Hexane:EtOAc, 19:1) to afford the dioxazoline product.

10ac (E)-but-2-en-1-yl 2-(5-methyl-5-phenyl-1,4,2-dioxazol-3-yl)benzoate

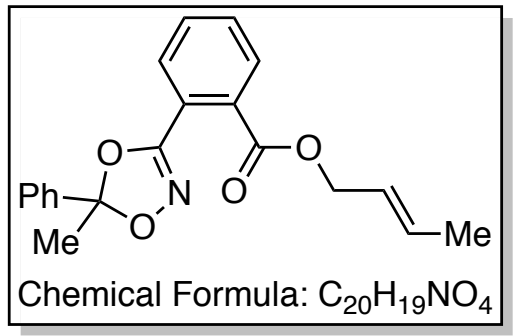

$\mathrm{Y}=38 \%$ Colorless Oil. $\mathrm{R}_{\mathrm{f}}=0.64$ (4:1 Hexanes:EtOAc).

${ }^{1}$ H NMR $(500 \mathrm{MHz}$, Chloroform- $d$ ) $\delta 7.80-7.74(\mathrm{~m}, 1 \mathrm{H}), 7.71-7.65(\mathrm{~m}, 1 \mathrm{H}), 7.62-7.58(\mathrm{~m}$, $2 \mathrm{H}), 7.56-7.50(\mathrm{~m}, 2 \mathrm{H}), 7.44-7.37(\mathrm{~m}, 3 \mathrm{H}), 5.81$ (dqt, $J=15.3,6.4,1.2 \mathrm{~Hz}, 1 \mathrm{H}), 5.59$ (dtq, $J$ $=14.8,6.5,1.6 \mathrm{~Hz}, 1 \mathrm{H}), 4.68(\mathrm{ddt}, J=12.3,6.5,1.2 \mathrm{~Hz}, 1 \mathrm{H}), 4.61(\mathrm{ddt}, J=12.2,6.5,1.1 \mathrm{~Hz}$, $1 \mathrm{H}), 2.01(\mathrm{~s}, 3 \mathrm{H}), 1.71(\mathrm{dq}, J=6.5,1.2 \mathrm{~Hz}, 3 \mathrm{H})$.

${ }^{13}$ C NMR (126 MHz, $\left.\mathrm{CDCl}_{3}\right) \delta 166.96,158.13,140.15,132.41,132.01,131.28,131.24,129.99$, $129.63,129.30,128.57,125.22,124.85,122.70,116.05,66.62,25.70,17.98$.

IR(neat) $2973,1726,1282,1261,1121,910,759,697 \mathrm{~cm}^{-1}$

LRMS m/z (ESI APCI) calculated for $\mathrm{C}_{20} \mathrm{H}_{19} \mathrm{NO}_{4}[\mathrm{M}+\mathrm{H}] 338.1$, found 338.1 

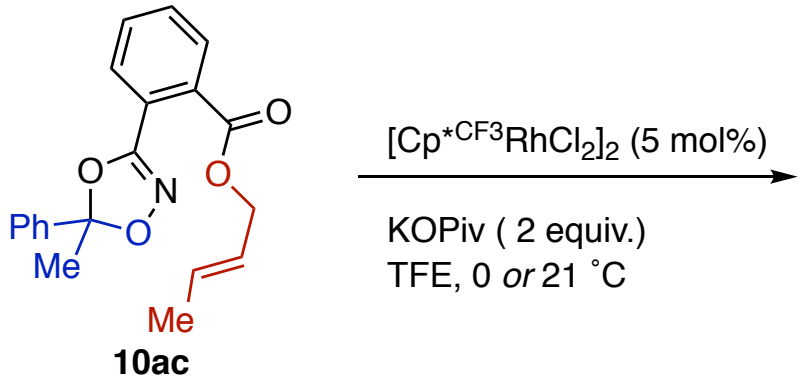<smiles>C[C@H]1C(CO)C1C(=O)c1ccccc1</smiles>

3ac not observed

Figure 27.

Dioxazoline 10ac $(0.12 \mathrm{mmol})$, catalyst $\left[\mathrm{Cp}^{* \mathrm{CF}^{3}} \mathrm{RhCl}_{2}\right]_{2}(5 \mathrm{~mol} \%, 0.006 \mathrm{mmol}, 4.4 \mathrm{mg})$, and KOPiv (2 equiv., $0.24 \mathrm{mmol}, 33.6 \mathrm{mg}$ ) were weighed in a 1-dram vial with a magnetic stirbar. Cooled (or room temperature) TFE $(0.2 \mathrm{M}, 600 \mu \mathrm{L})$ was added. The vial was sealed with a screw-cap and placed in an aluminium block cooled to $0{ }^{\circ} \mathrm{C}$ surrounded by ice in an insulated box (or placed on a stir plate at room temperature) and stirred for 16 hours. TFE was removed by rotary evaporation and the residue was taken up in EtOAc and filtered through a silica plug flushing with EtOAc. The filtrate was concentrated to $\sim 1 \mathrm{~mL}$ and transferred to a 1.5-dram vial where the solution was partitioned with the addition of $\mathrm{H}_{2} \mathrm{O}$. The aqueous layer was extracted three times with EtOAc and the combined organic extracts were filtered through a pad of celite ${ }^{\circledR}$ and $\mathrm{Na}_{2} \mathrm{SO}_{4}$ then concentrated. Yield and diastereoselectivity were determined by crude ${ }^{1} \mathrm{H}-$ NMR.

Observation of opened-phthalimide ring by ${ }^{1} H$-NMR spectroscopy<smiles>[2H]C([2H])=C(ON1C(=O)c2ccccc2C1=O)c1c([2H])c([2H])c([2H])c([2H])c1[2H]</smiles>

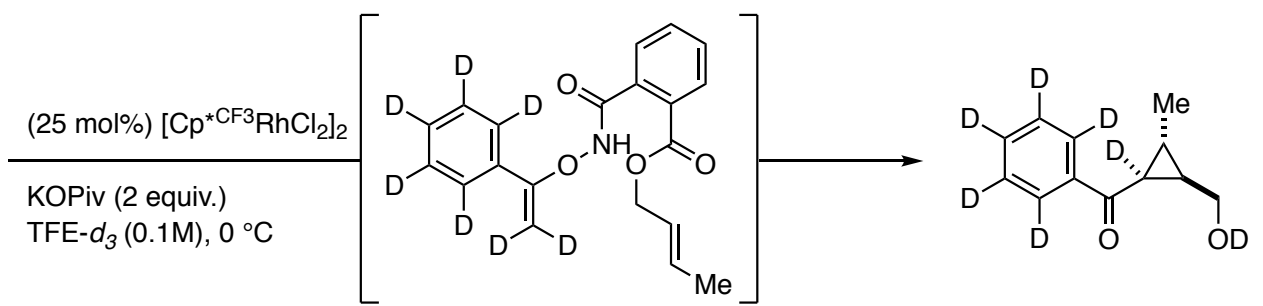

Figure 28.

$\mathrm{N}$-enoxyphthalimide 1aa' $(0.06 \mathrm{mmol})$ and KOPiv (2 equiv., $0.12 \mathrm{mmol})$ were weighed in a 1 dram vial with a magnetic stirbar. TFE- $d_{3}(0.1 \mathrm{M}, 600 \mathrm{~mL})$ was added followed by allylic alcohol $2 c$ ( 1.7 equiv., $0.10 \mathrm{mmol}$ ). The vial was sealed with a screw-cap and stirred briefly and the solution transferred to an NMR tube and injected in the spectrometer set to $273 \mathrm{~K}$. 

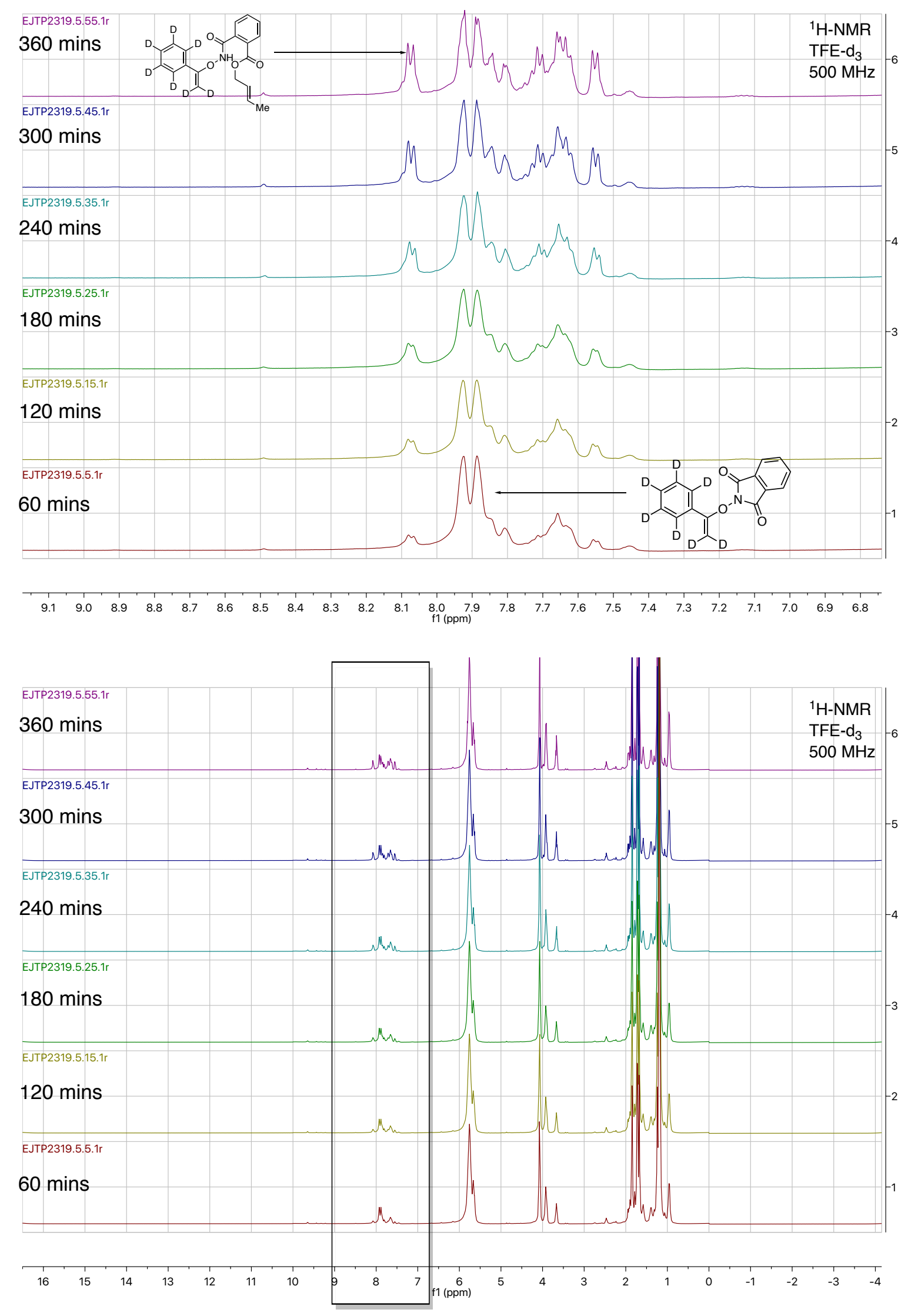
V Model for Diastereoselectivity<smiles>[R]C=CC([R])O</smiles>

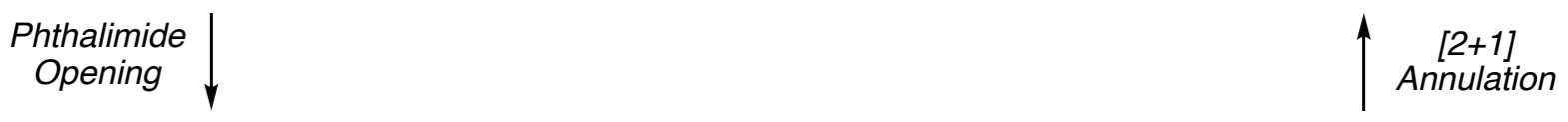<smiles>[R]C=CC([R7])OC(=O)c1ccccc1C(=O)NOC(=C)[Al]</smiles>

\section{- Conformational analysis of the proposed Rh-carbenoid- the diastereo-determining step of the reaction}

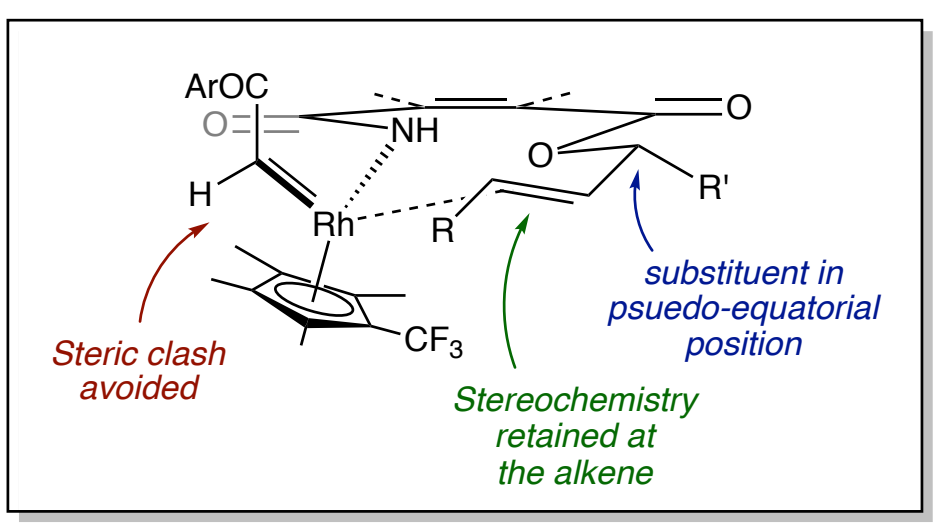

\section{-Major Diastereomer}

-Stereochemistry retained at alkene

-Steric clash avoided with $\mathrm{Cp}$

-Substituent is equatorial<smiles>[R]C(O)[C@H]1[C@H]2C(=O)[C@@H]1[C@@H]2[Z7]</smiles><smiles>[R]C(O)[C@H]1C(C(=O)[Al])[C@H]1I</smiles>

-Minor Diastereomer

-Stereochemistry retained at alkene

-Steric clash with $\mathrm{Cp}$

-Substituent is equatorial

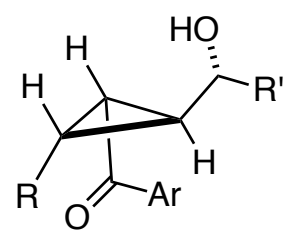<smiles>[R]C(O)[C@@H]1[C@H](I)[C@@H]1C(=O)[Al]</smiles>

-Minor Diastereomer

-Stereochemistry retained at alkene -Steric clash avoided with $\mathrm{Cp}$

-Substituent is axial<smiles></smiles><smiles>[R]C(O)C1C([2H])C1C(=O)[Al]</smiles>

-Minor Diastereomer

-Stereochemistry retained at alkene -Steric clash with Cp

-Substituent is axial<smiles>[R]C(O)C1CC2CC1C([R])C2=[Bi]</smiles><smiles>[R]C(O)[C@@H]1[C@H]([2H])[C@@H]1C(=O)[Al]</smiles> 


\section{X-Ray Data}

Single crystal X-ray diffraction. Data for all compounds was collected on an Agilent SuperNova diffractometer using mirror-monochromated $\mathrm{Cu} K \alpha$ radiation. Data collection, integration, scaling (ABSPACK) and absorption correction (face-indexed Gaussian integration ${ }^{13}$ or numeric analytical methods ${ }^{14}$ ) were performed in CrysAlisPro. ${ }^{15}$ Structure solution was performed using ShelXT ${ }^{16}$. Subsequent refinement was performed by full-matrix least-squares on $\mathrm{F}^{2}$ in ShelXL. ${ }^{17}$ Olex $2^{18}$ was used for viewing and to prepare CIF files. PLATON ${ }^{19}$ was used extensively for CheckCIF. ORTEP graphics were prepared in CrystalMaker. ${ }^{20}$ Thermal ellipsoids are rendered at the $50 \%$ probability level.

A solution of EJTP2213_B_pure in $\mathrm{CHCl}_{3} /$ hexanes was slowly evaporated to afford long, colorless needles. Part of a crystal $(.46 \times .06 \times .04 \mathrm{~mm})$ was separated carefully, mounted on a glass fiber with Paratone oil, and cooled to $100 \mathrm{~K}$ on the diffractometer. Complete data were collected to $0.8 \AA$ A. 12900 reflections were collected (2666 unique, 2373 observed) with R(int) $5.9 \%$ and $\mathrm{R}$ (sigma) $4.2 \%$ after Gaussian absorption and beam profile correction (maximum correction factor 1.46).

The space group was assigned tentatively as I2/a based on the systematic absences. Using ShelXT, the structure solved readily in I2/a with 1 molecule in the asymmetric unit. All non-H atoms were located in the initial solution and refined anisotropically with no restraints. The O-H hydrogen was located in a difference map and refined with unrestrained coordinates and isotropic ADP. C-H hydrogens were placed in calculated positions and refined with riding coordinates and ADPs.

The final refinement (2666 data, 0 restraints, 176 parameters) converged with $R_{1}\left(F_{o}>4 \sigma\left(F_{0}\right)\right)=$ $4.6 \%, \mathrm{wR}_{2}=12.1 \%, \mathrm{~S}=1.04$. The largest Fourier features were 0.25 and $-0.20 \mathrm{e}^{-} \mathrm{A}^{-3}$.

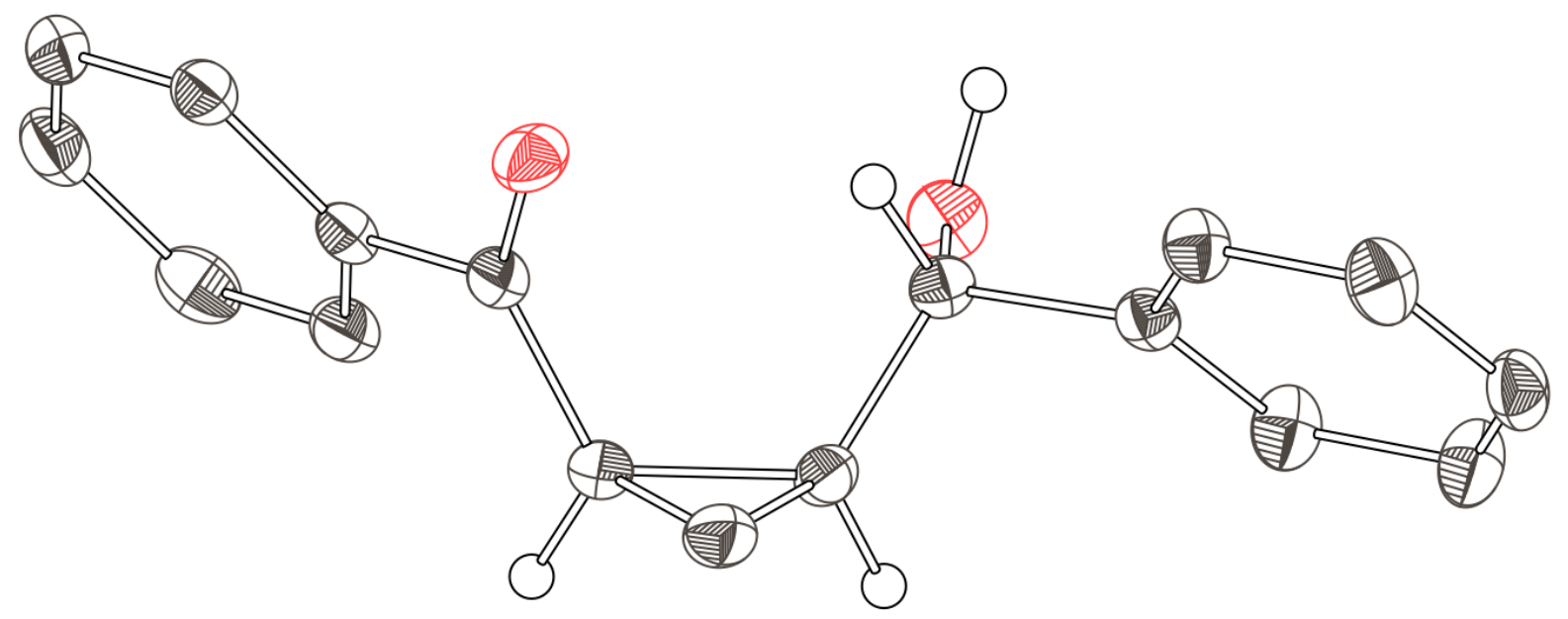

Molecular structure of EJTP2213_B_pure. The crystal is centrosymmetric and thus contains both enantiomers. 


\section{Compound EJTP2213_B_pure}

\begin{tabular}{|c|c|}
\hline Formula & $\mathrm{C}_{17} \mathrm{H}_{16} \mathrm{O}_{2}$ \\
\hline MW & 252.30 \\
\hline Space group & $\mathrm{I} 2 / \mathrm{a}$ \\
\hline$a(\AA)$ & $20.0490(6)$ \\
\hline$b(\AA)$ & $5.46156(14)$ \\
\hline$c(\AA)$ & $25.6163(8)$ \\
\hline$\alpha\left(^{\circ}\right)$ & 90 \\
\hline$\beta\left({ }^{\circ}\right)$ & $107.386(3)$ \\
\hline$\gamma\left({ }^{\circ}\right)$ & 90 \\
\hline$V\left(\AA^{3}\right)$ & $2676.80(14)$ \\
\hline $\mathbf{Z}$ & 8 \\
\hline$\rho_{\text {calc }}\left(\mathrm{g} \mathrm{cm}^{-3}\right)$ & 1.252 \\
\hline $\mathbf{T}(\mathbf{K})$ & 100 \\
\hline$\lambda(\AA)$ & 1.54184 \\
\hline $2 \theta_{\min }, 2 \theta_{\max }$ & 7,146 \\
\hline Nref & 12900 \\
\hline $\mathbf{R}($ int $), \mathbf{R}(\sigma)$ & $.0591, .0416$ \\
\hline$\mu\left(\mathrm{mm}^{-1}\right)$ & 0.642 \\
\hline Size (mm) & $.46 \times .06 \times .04$ \\
\hline $\mathbf{T}_{\max } / \mathbf{T}_{\min }$ & 1.46 \\
\hline Data & 2666 \\
\hline Restraints & 0 \\
\hline Parameters & 176 \\
\hline $\mathbf{R}_{1}$ (obs) & 0.0458 \\
\hline $\mathrm{wR}_{2}$ (all) & 0.1212 \\
\hline $\mathbf{S}$ & 1.036 \\
\hline
\end{tabular}

Peak, hole ( $\mathrm{e}^{-}$
$\left.\AA^{-3}\right)$
$0.25,-0.20$

\section{NMR Spectra}



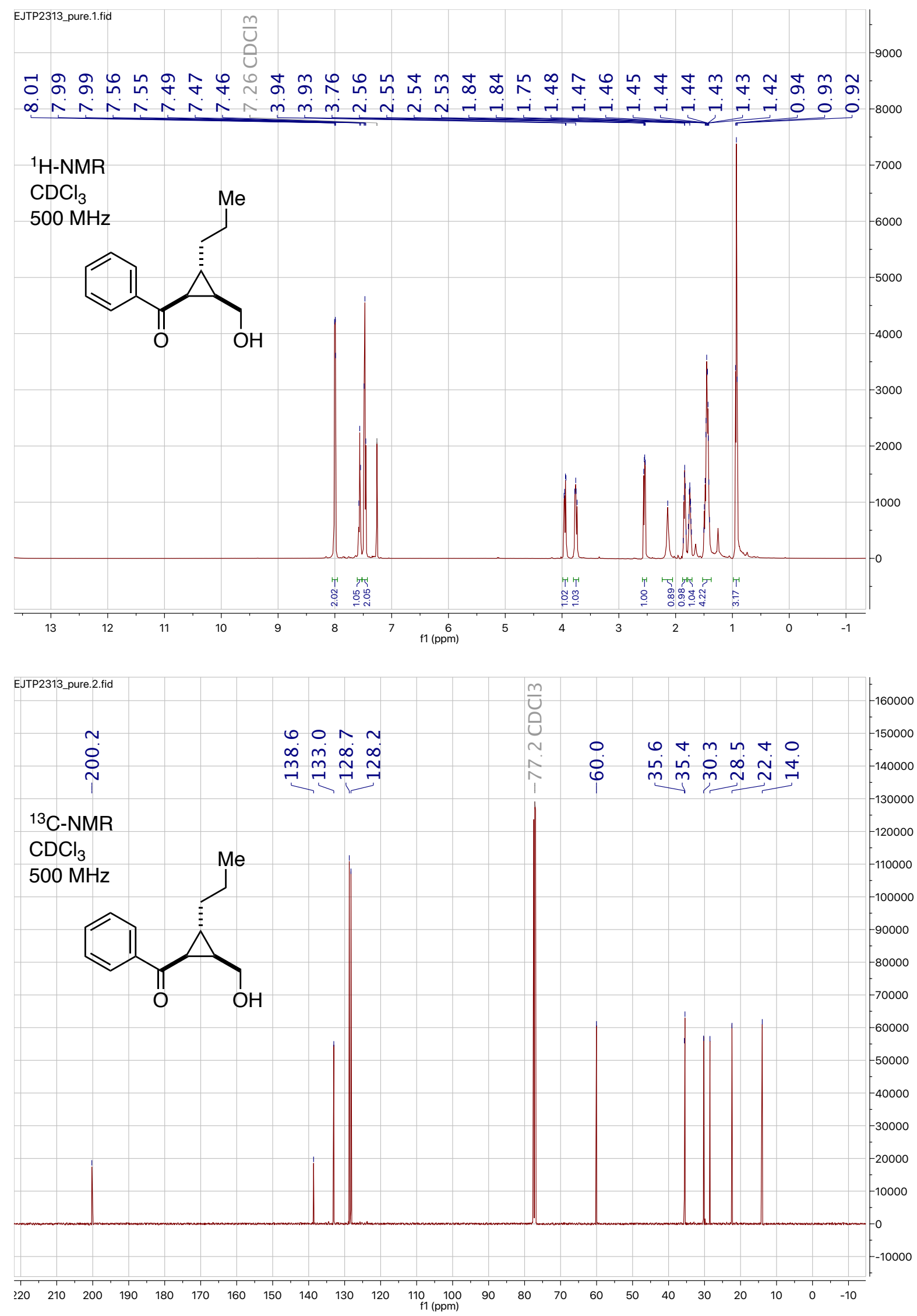

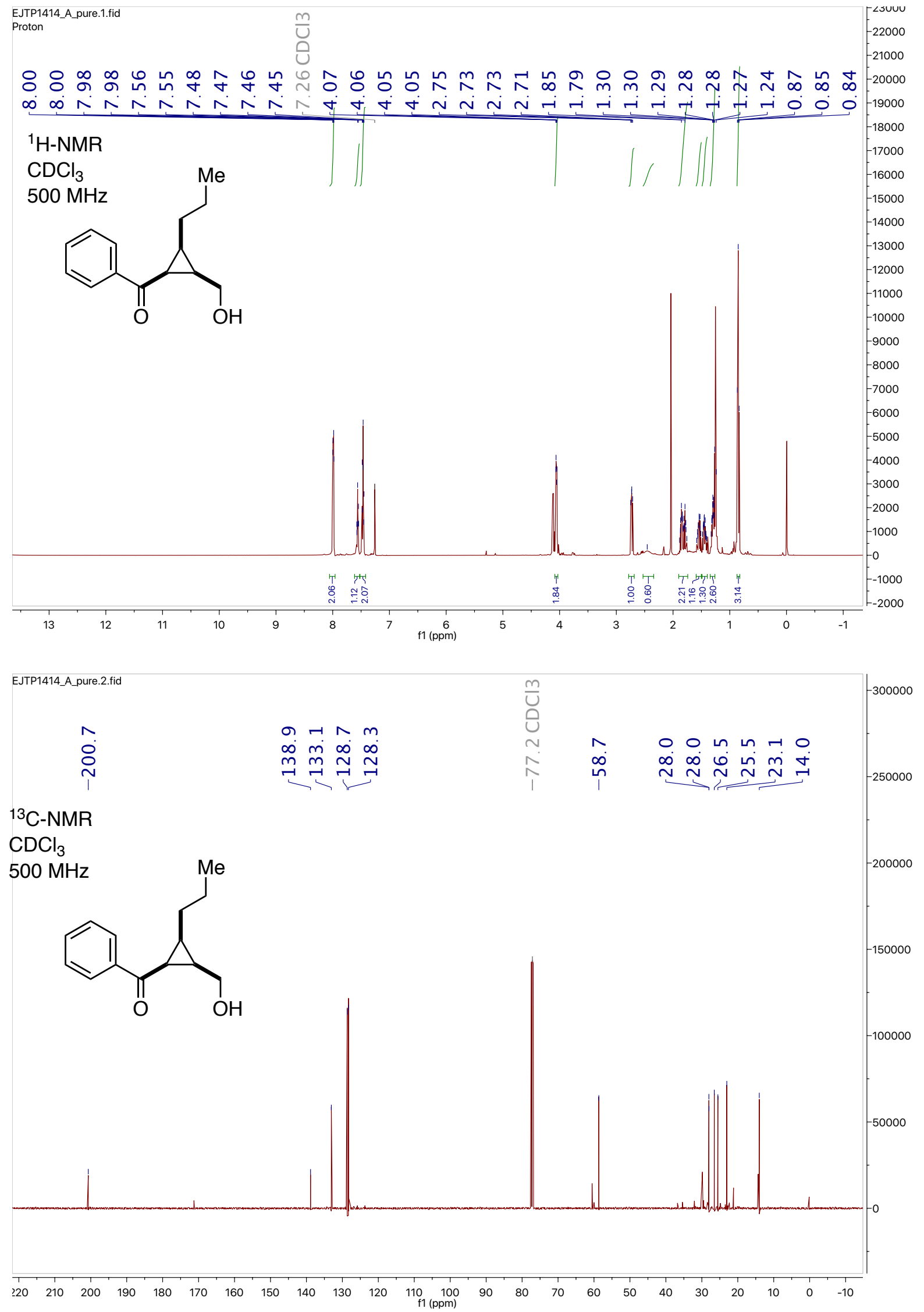


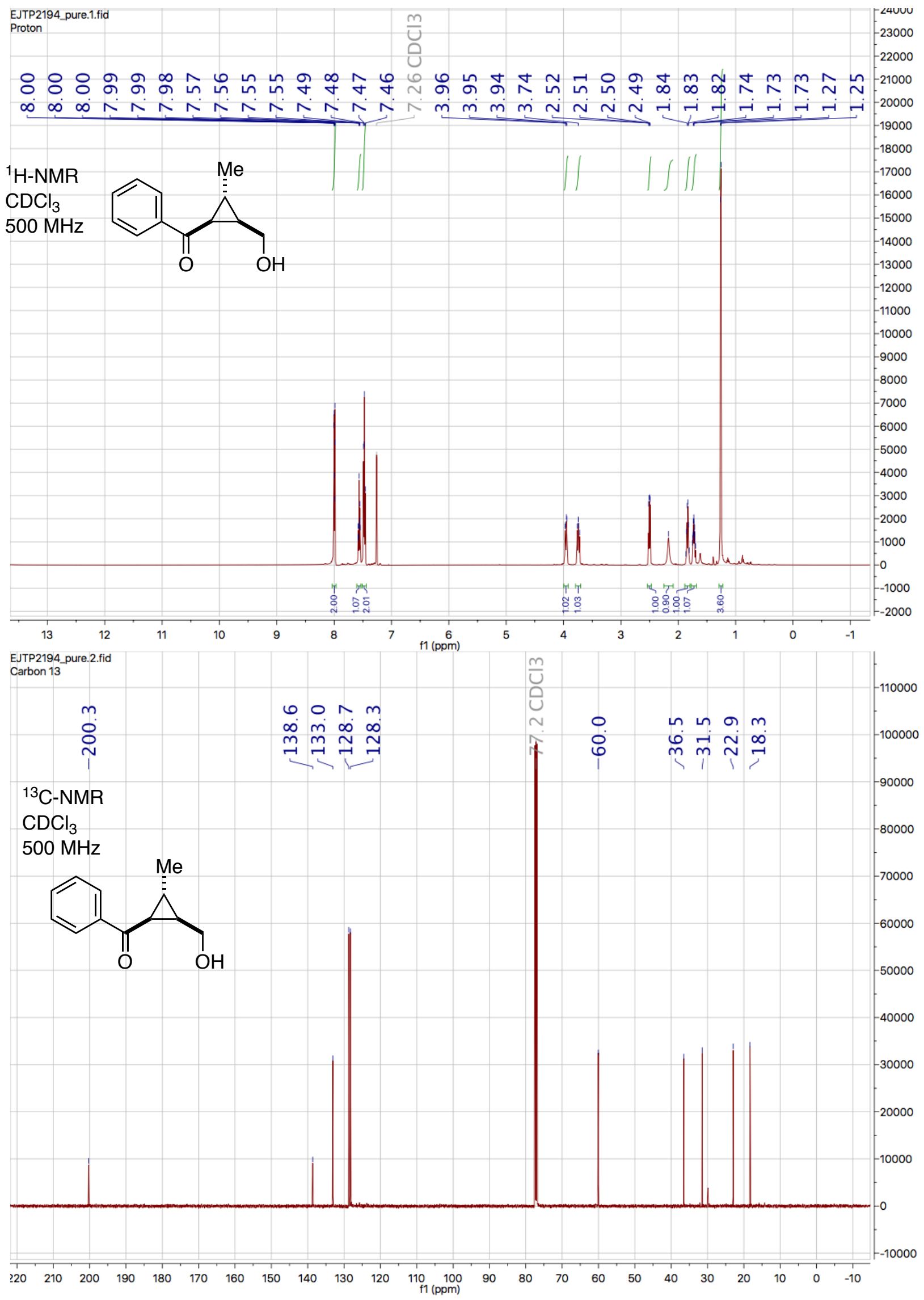



○ ने ने

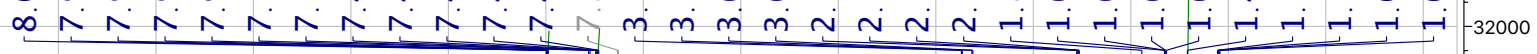
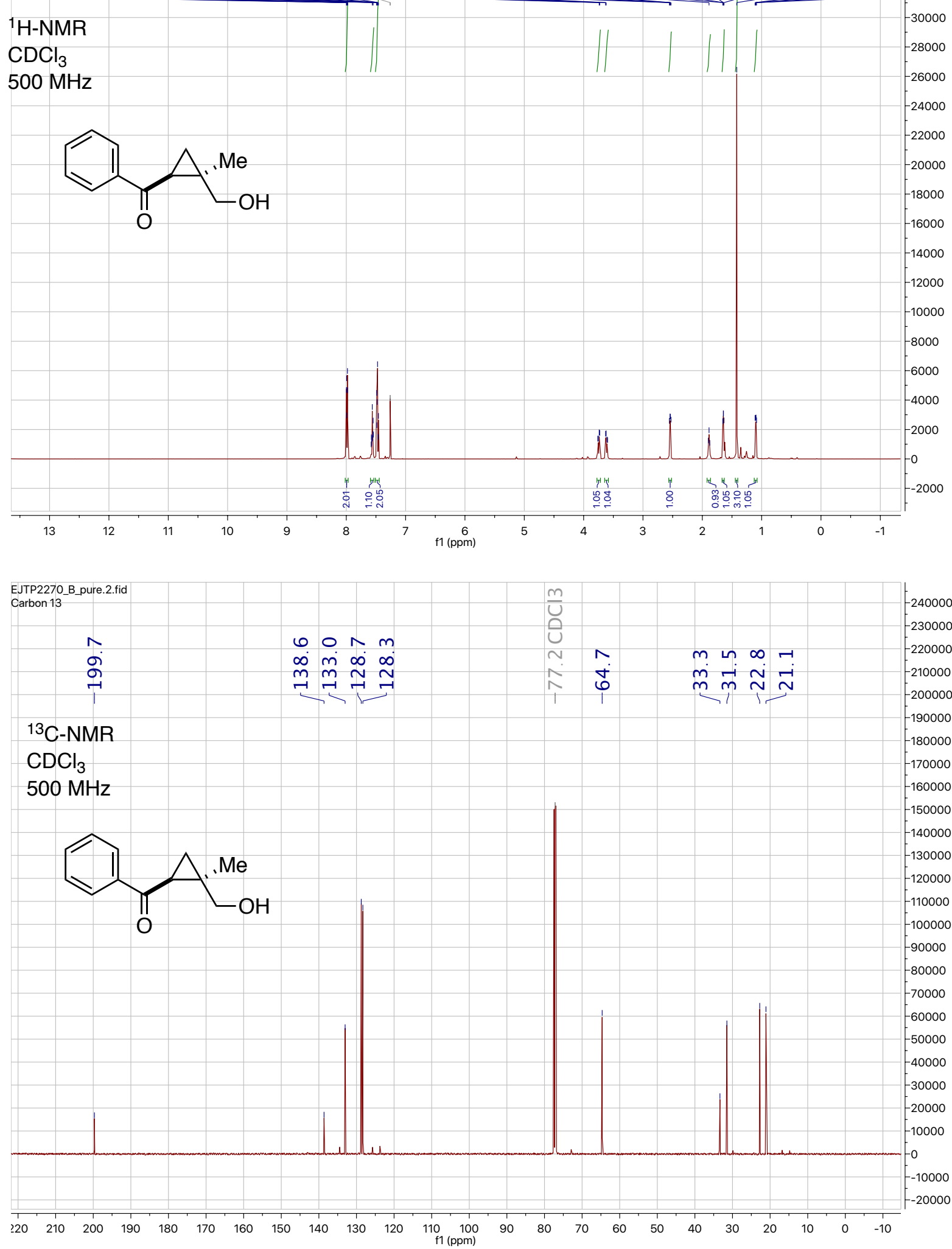

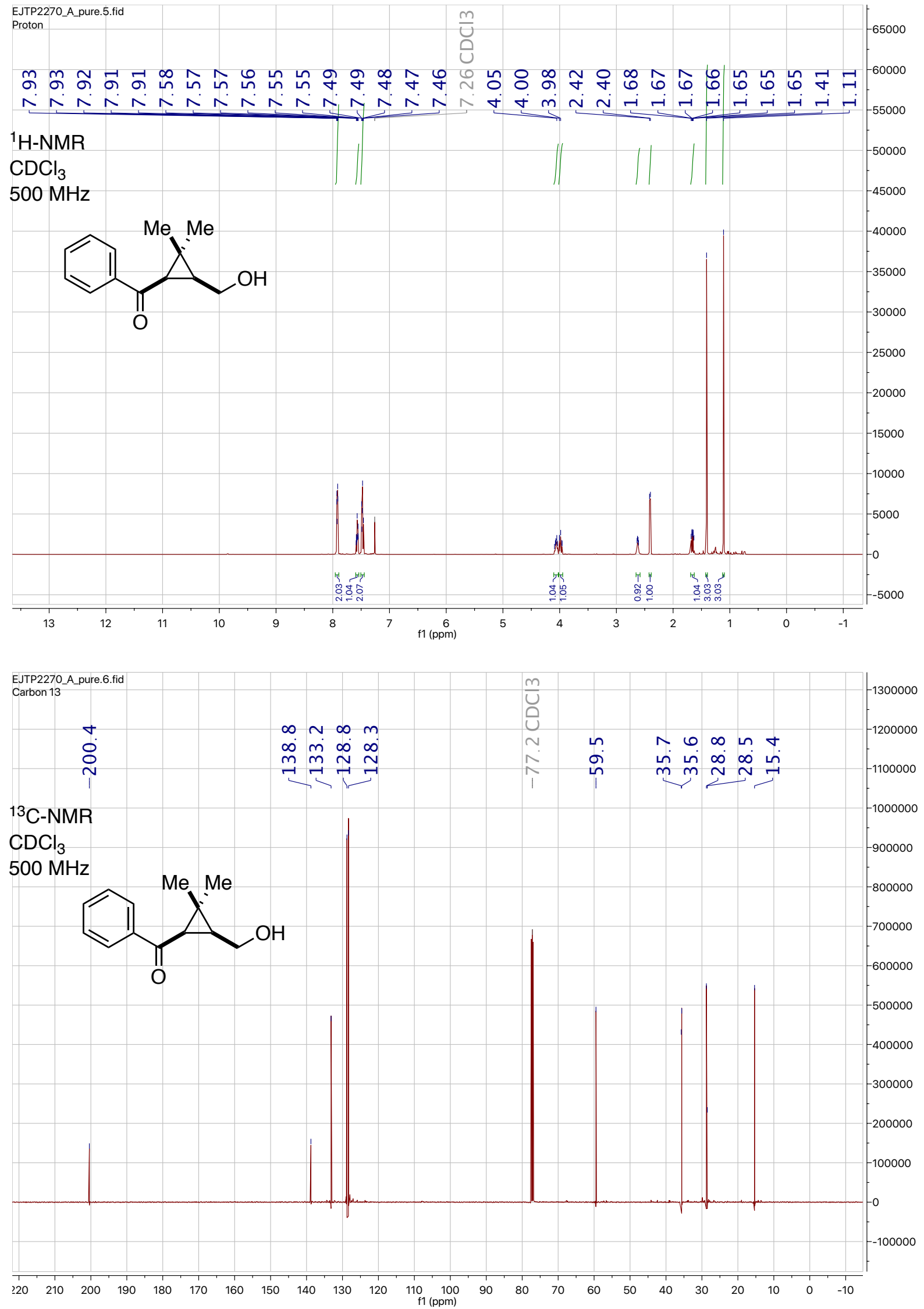


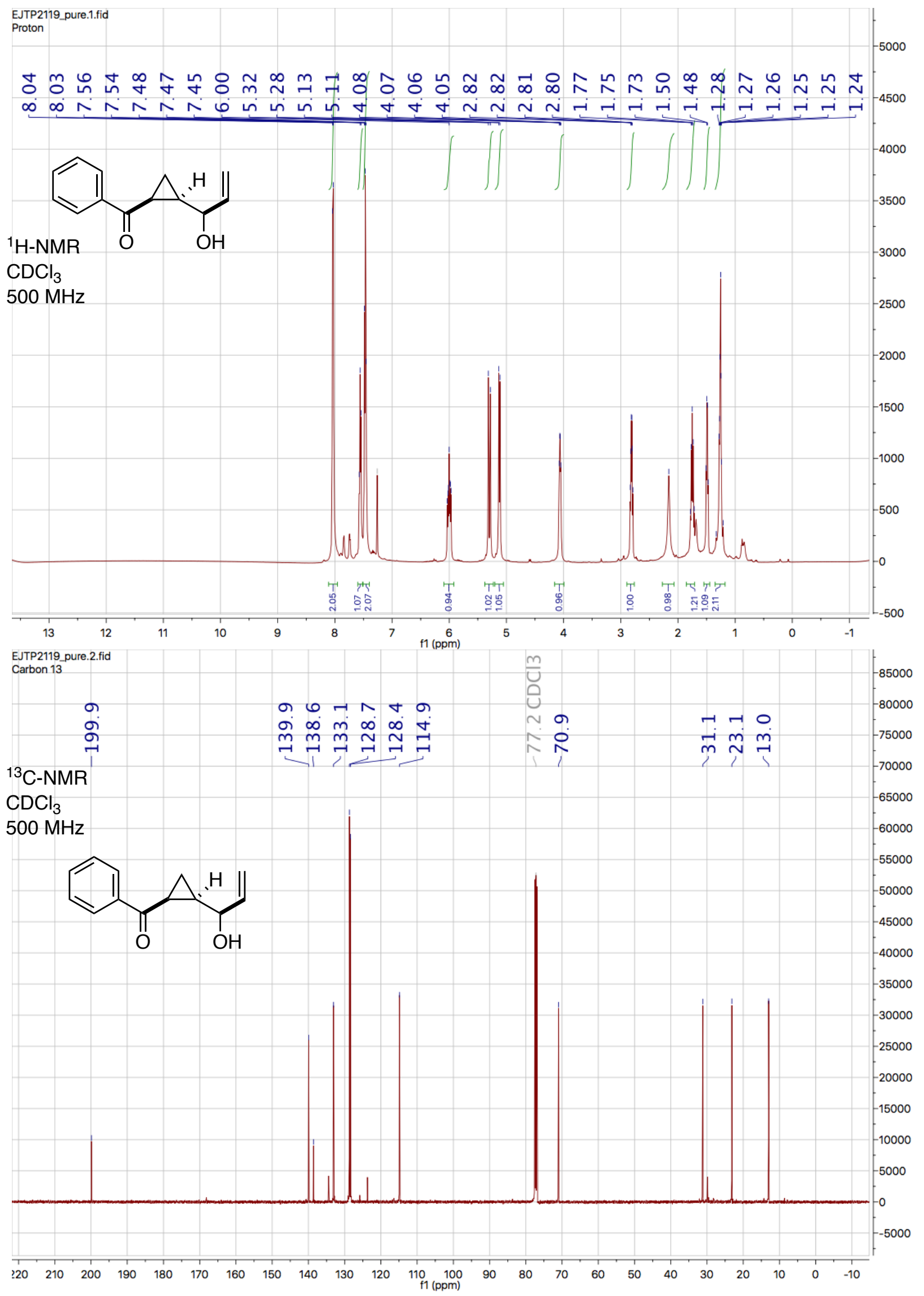



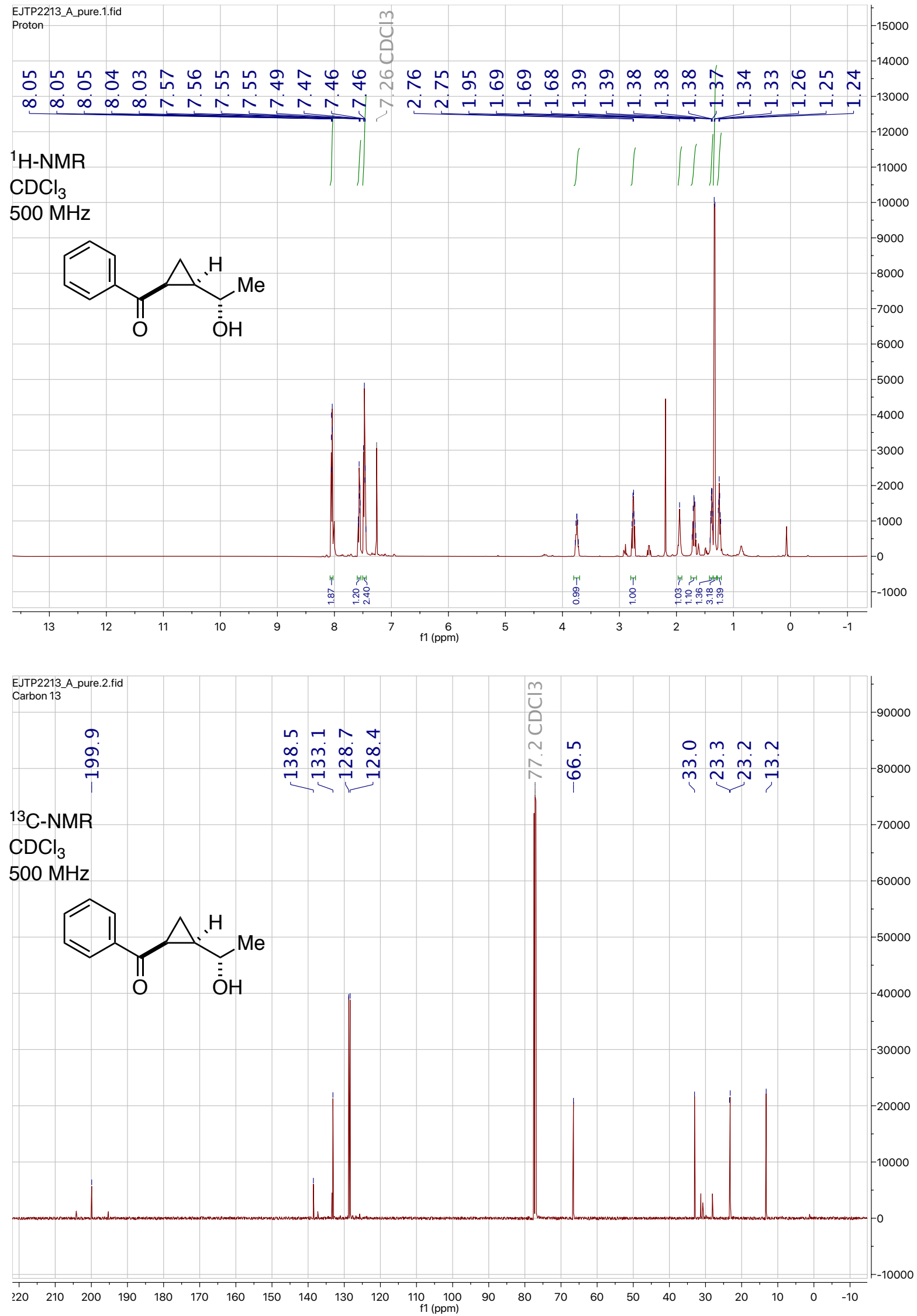

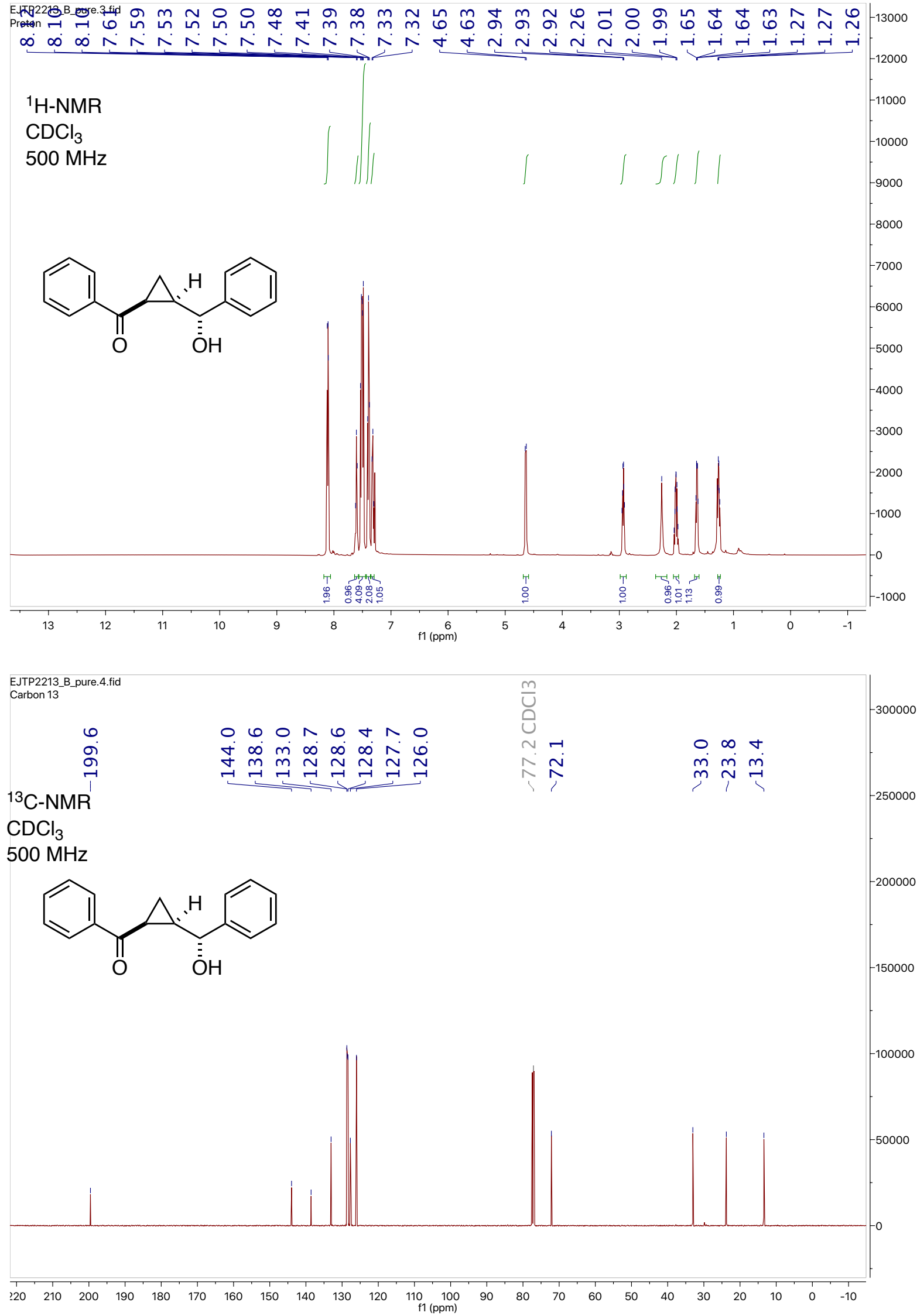


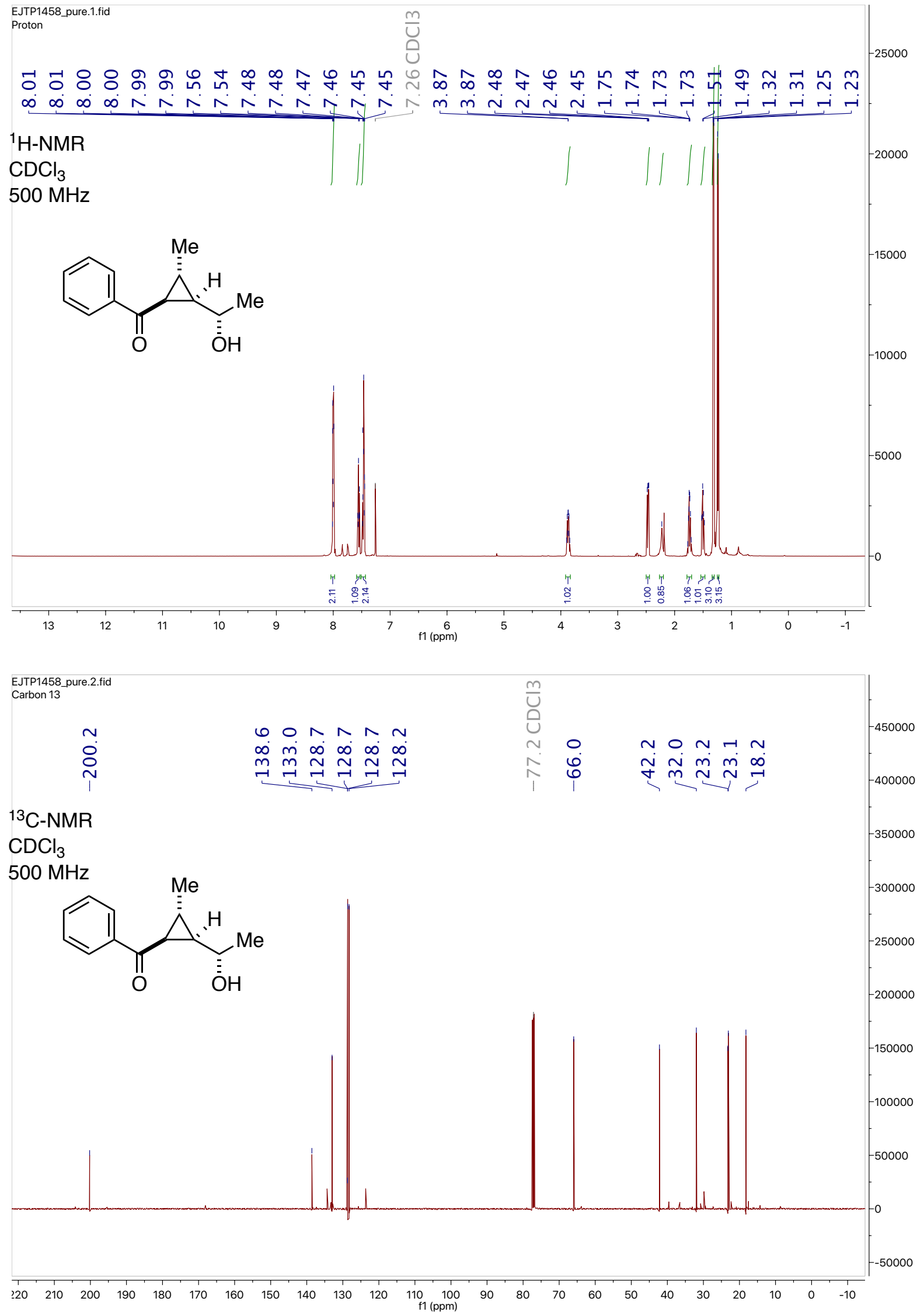



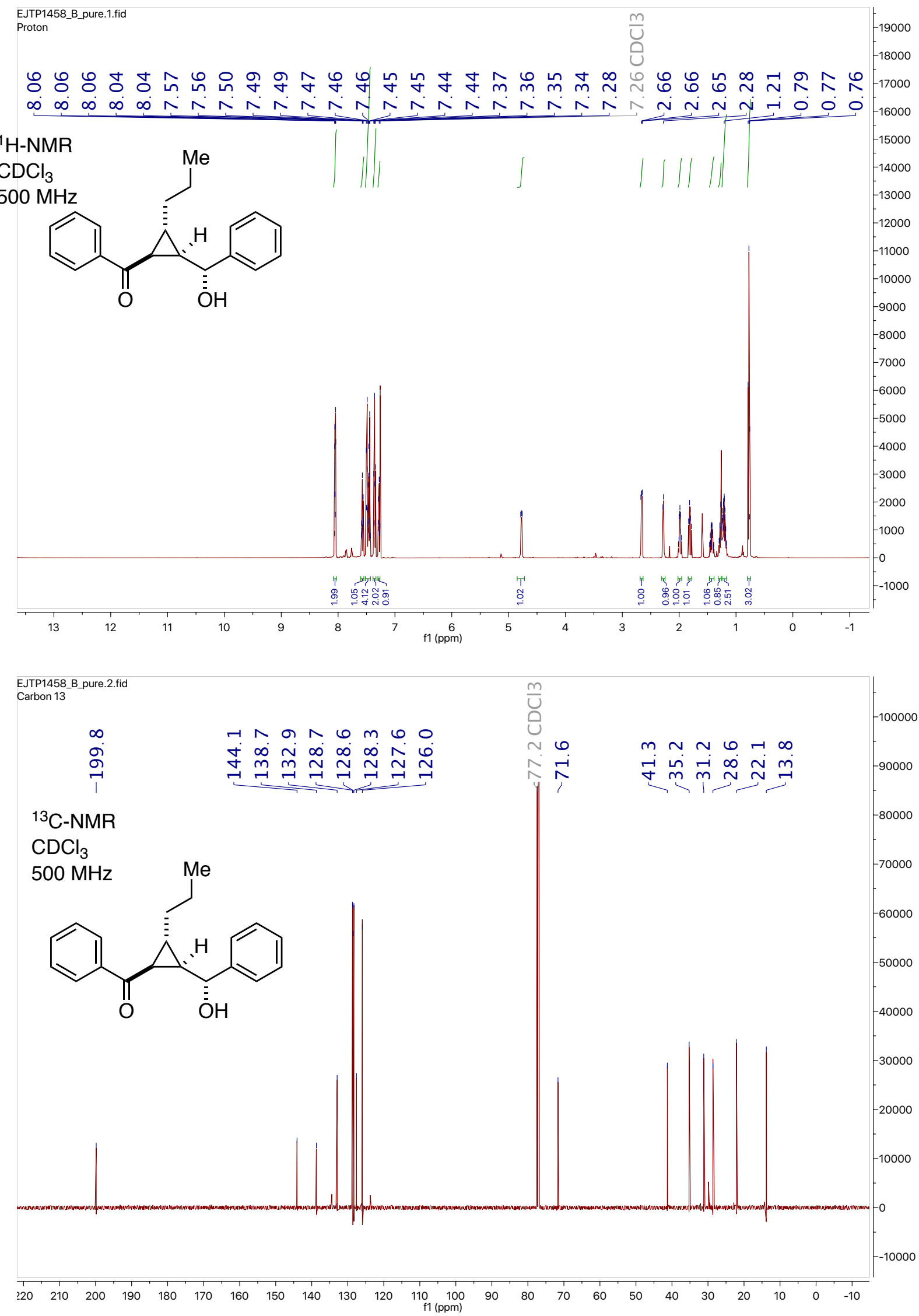


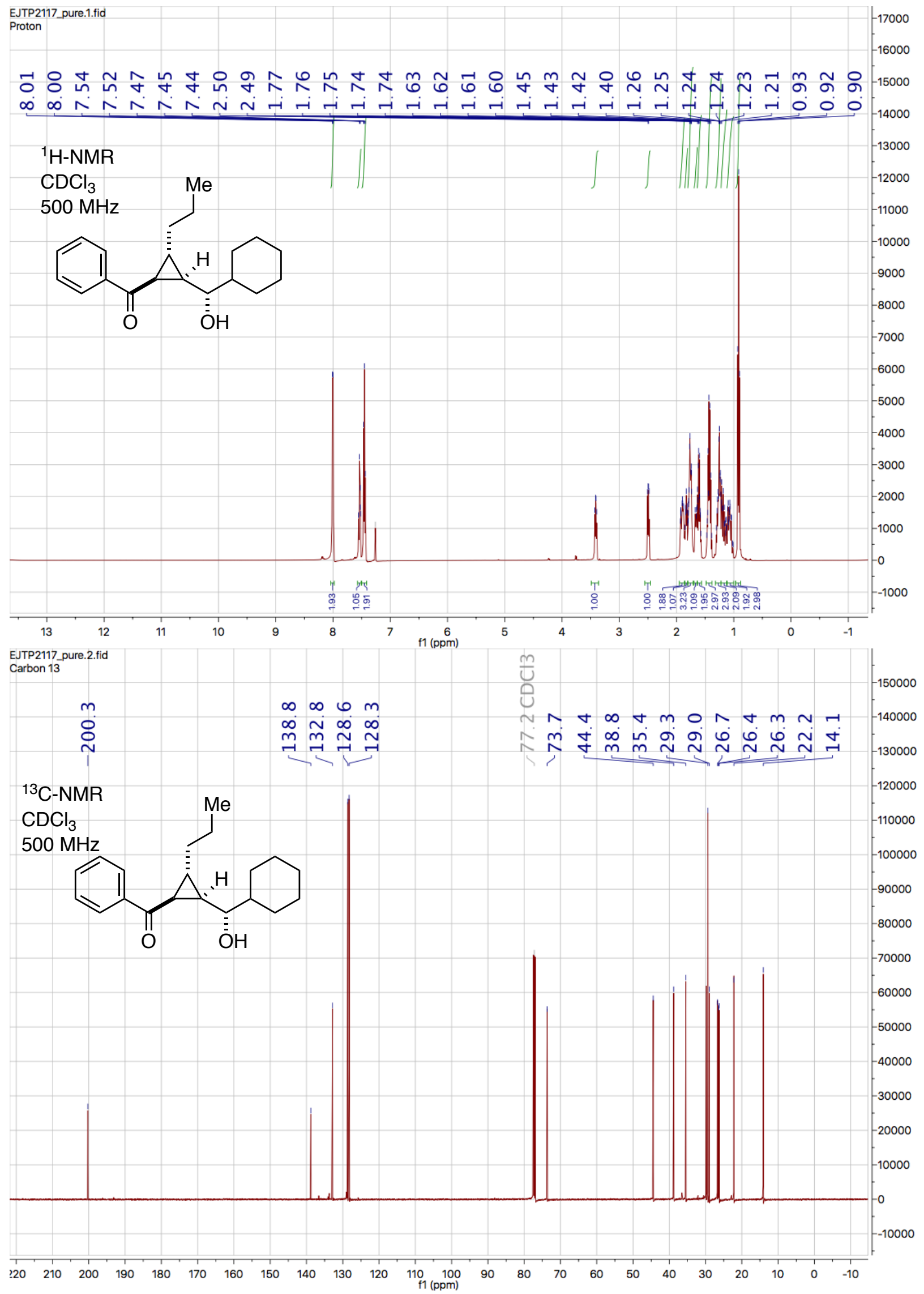




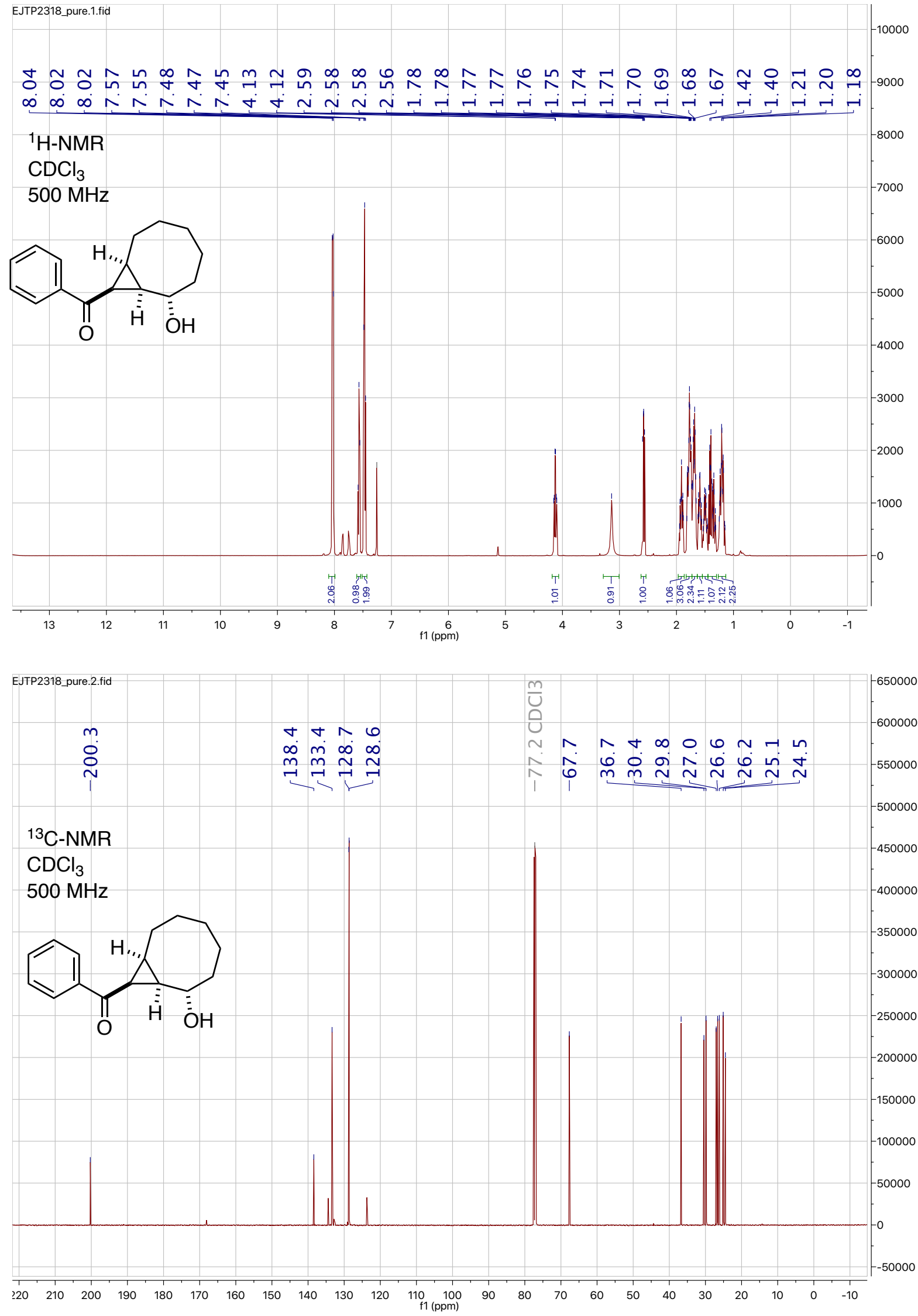



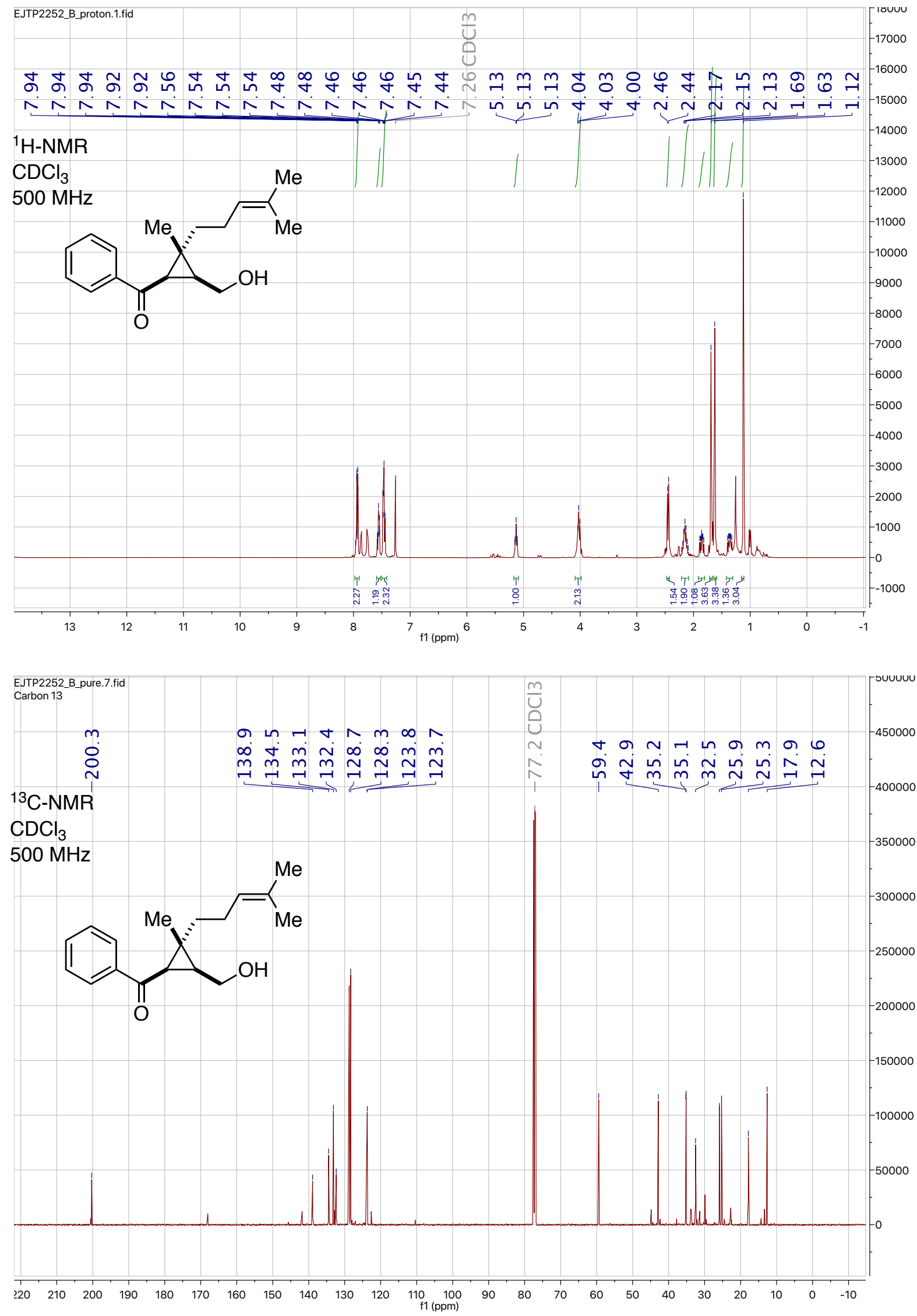

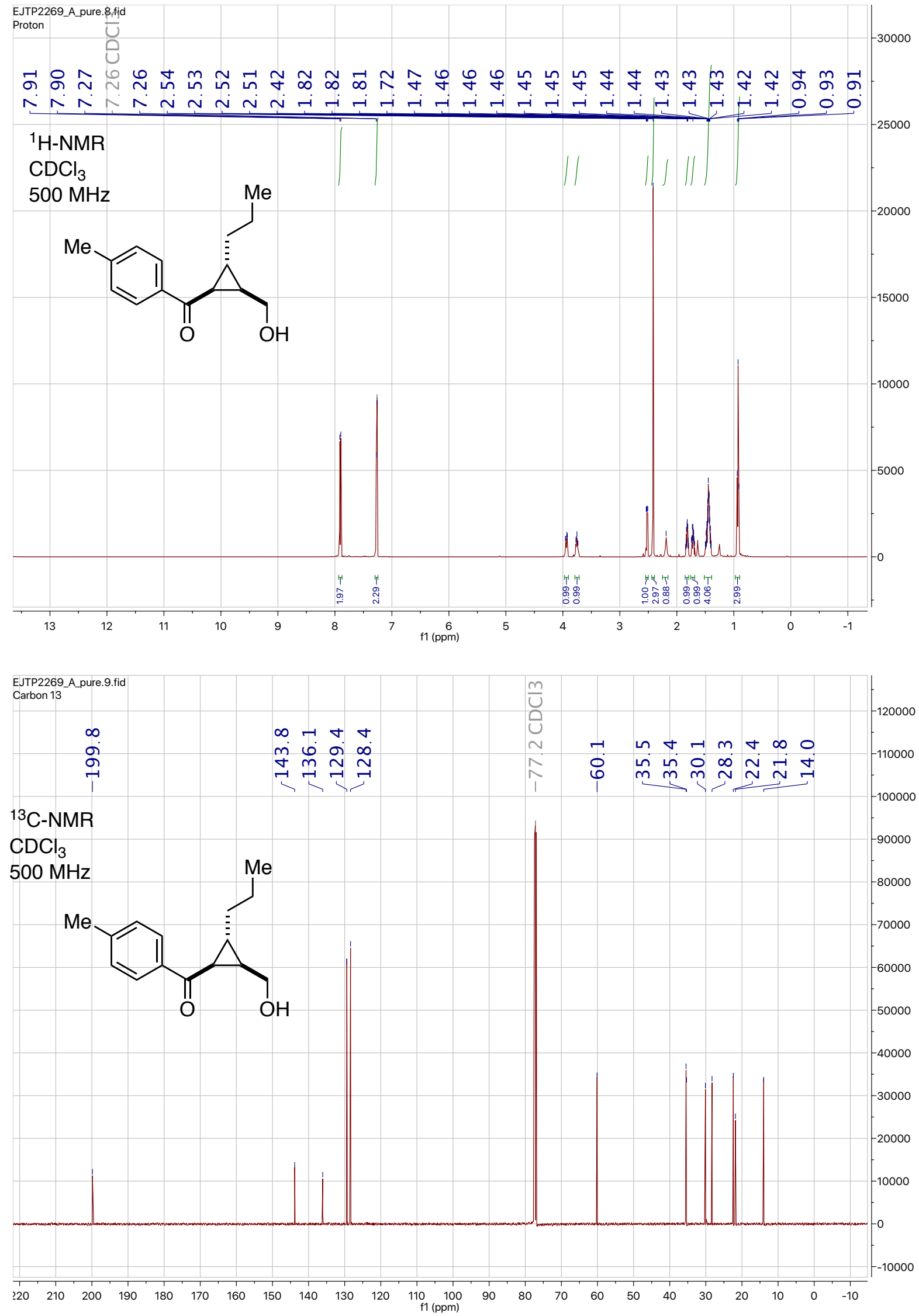

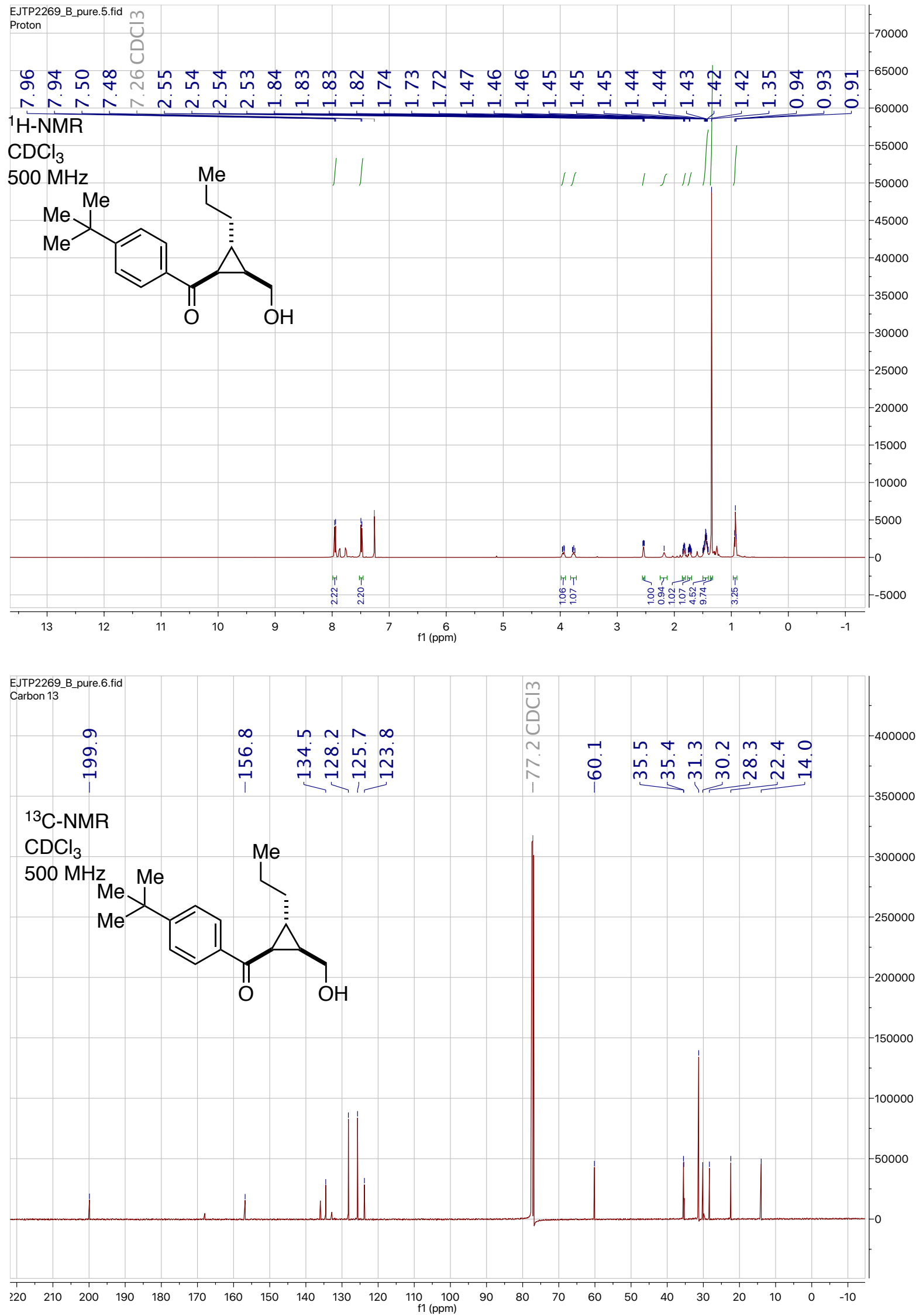


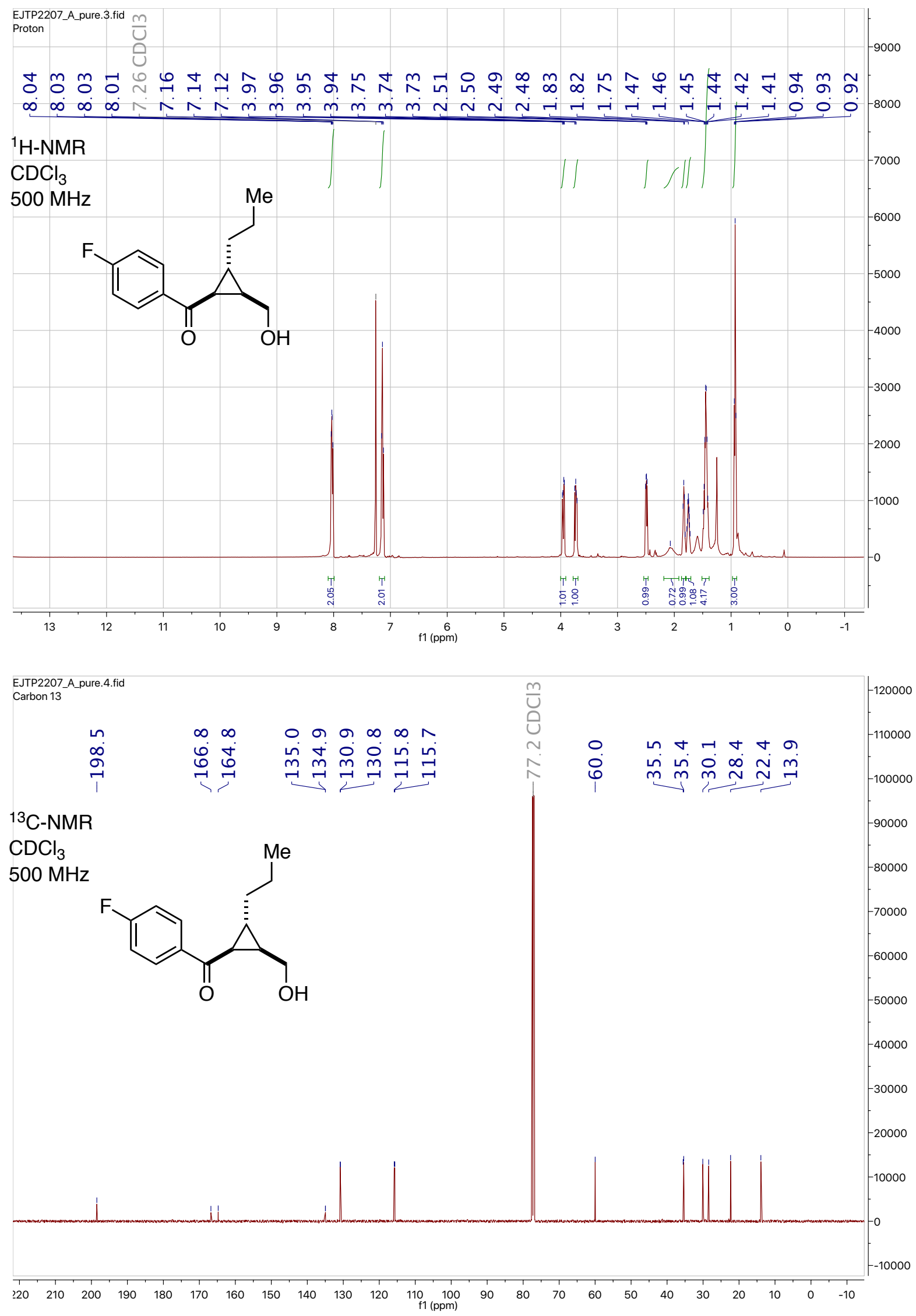




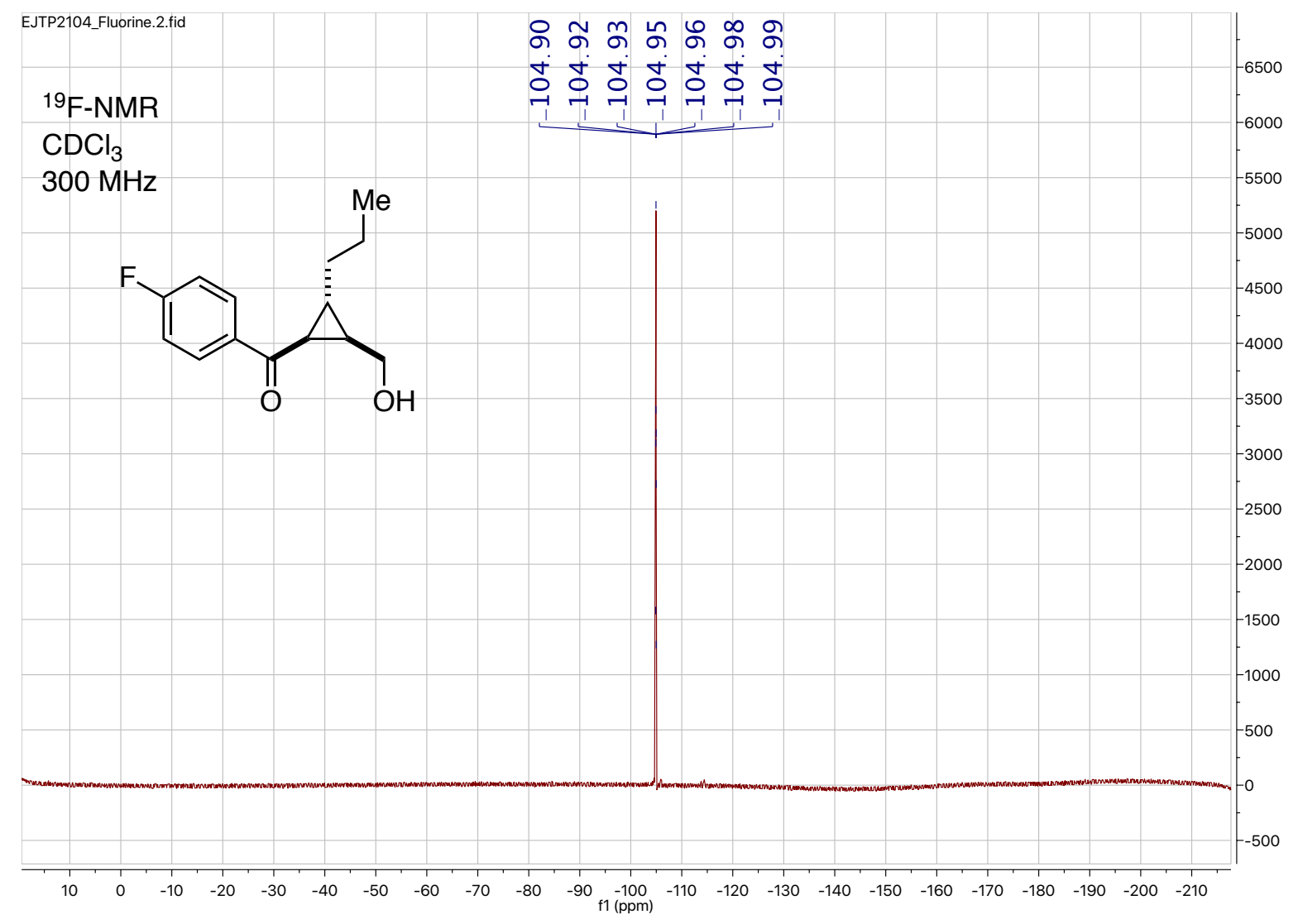




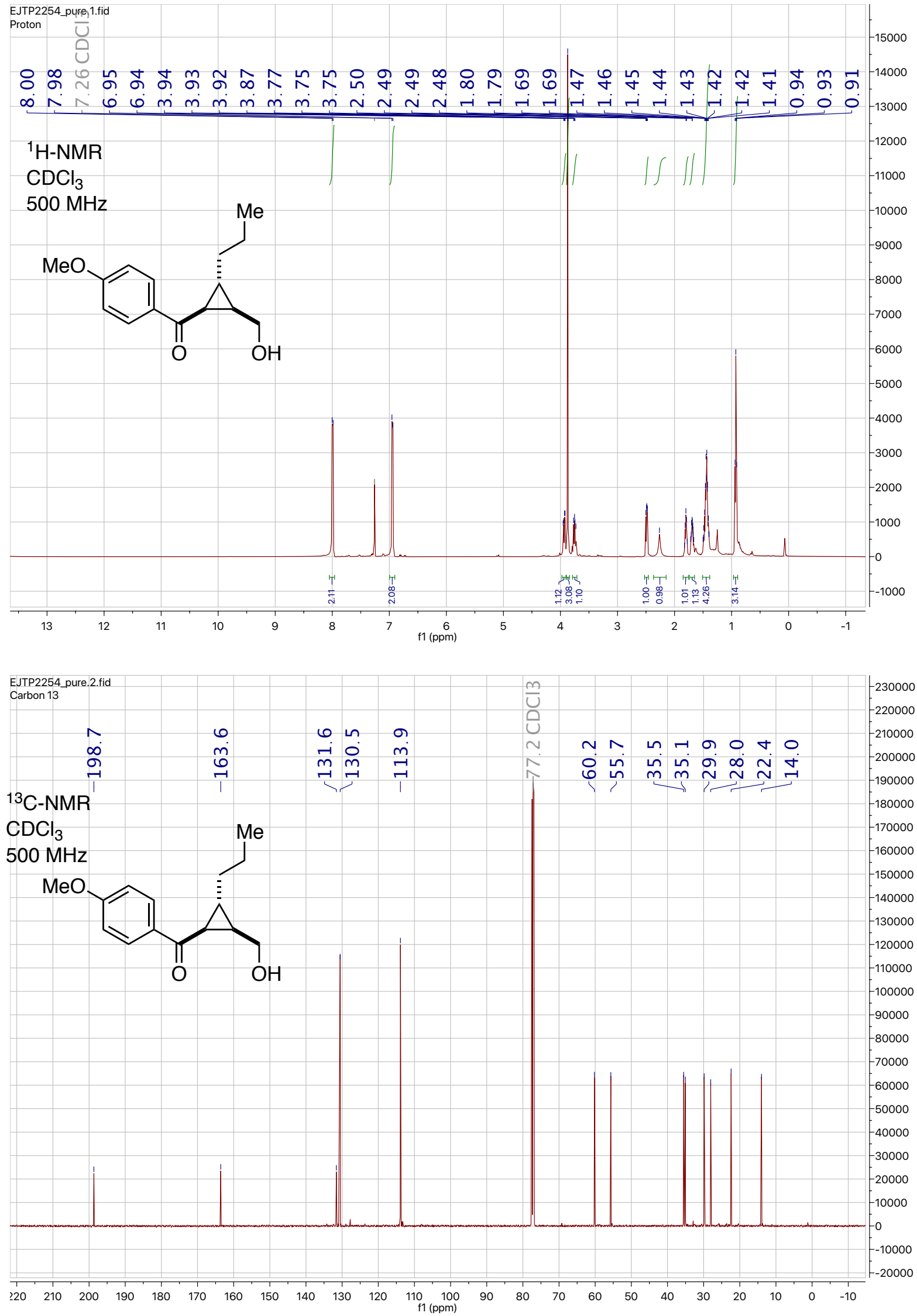




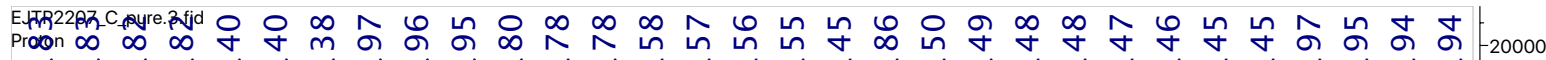

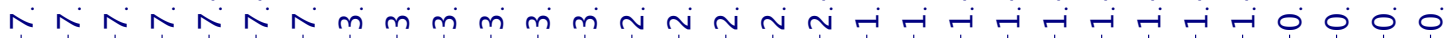

\section{${ }^{1} \mathrm{H}-\mathrm{NMR}$ \\ $\mathrm{CDCl}_{3}$}

$500 \mathrm{MHz}$
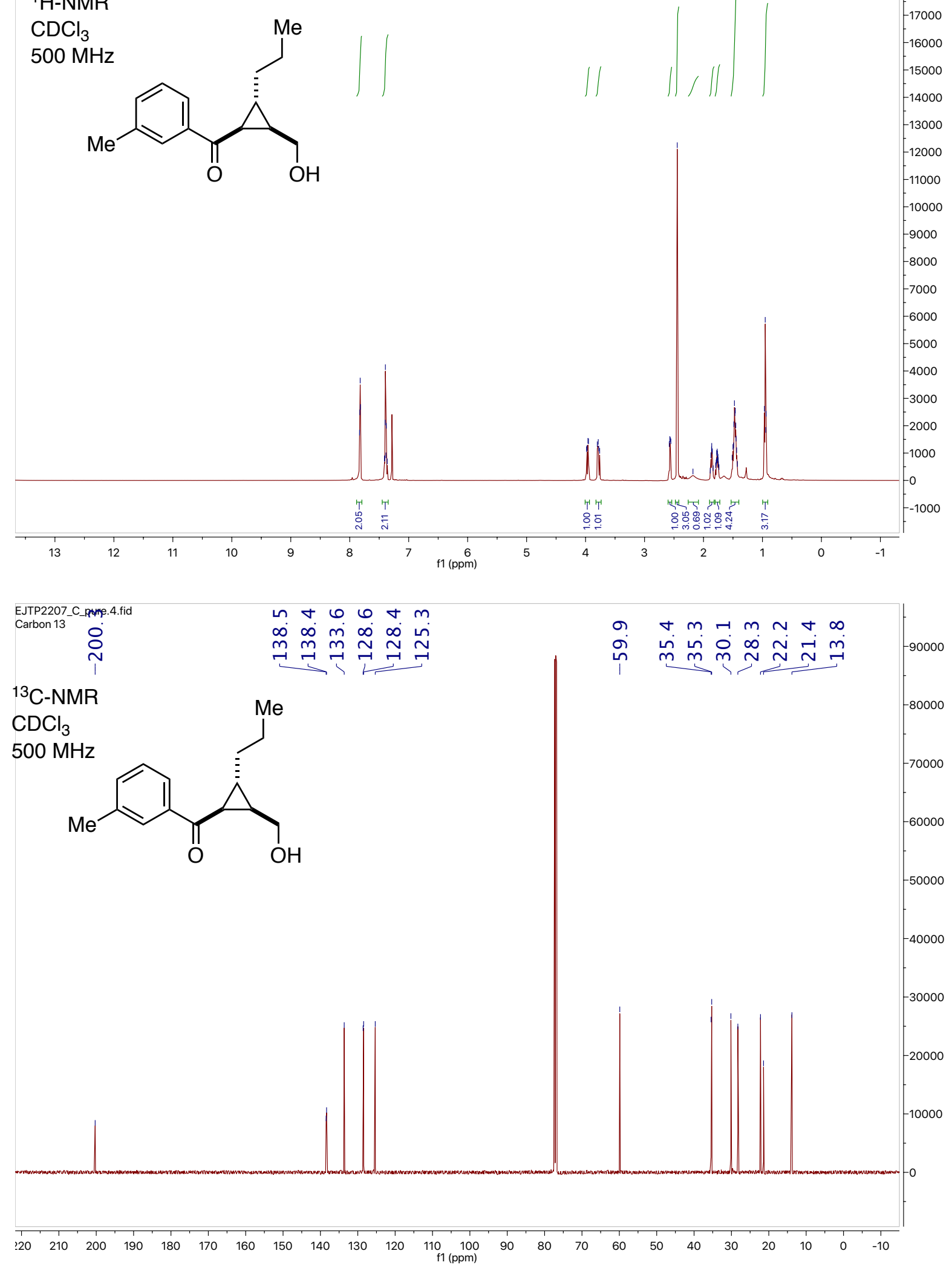


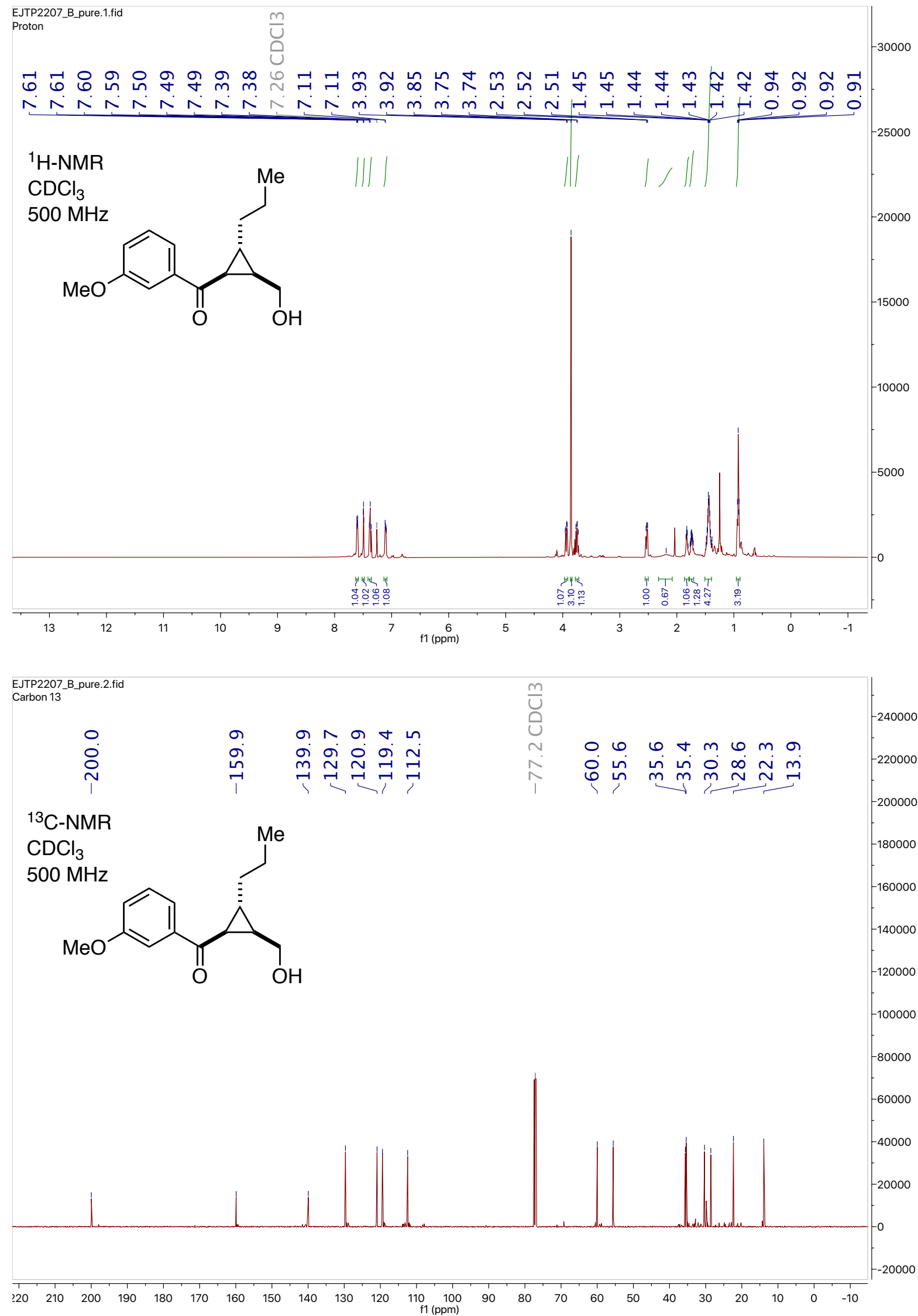



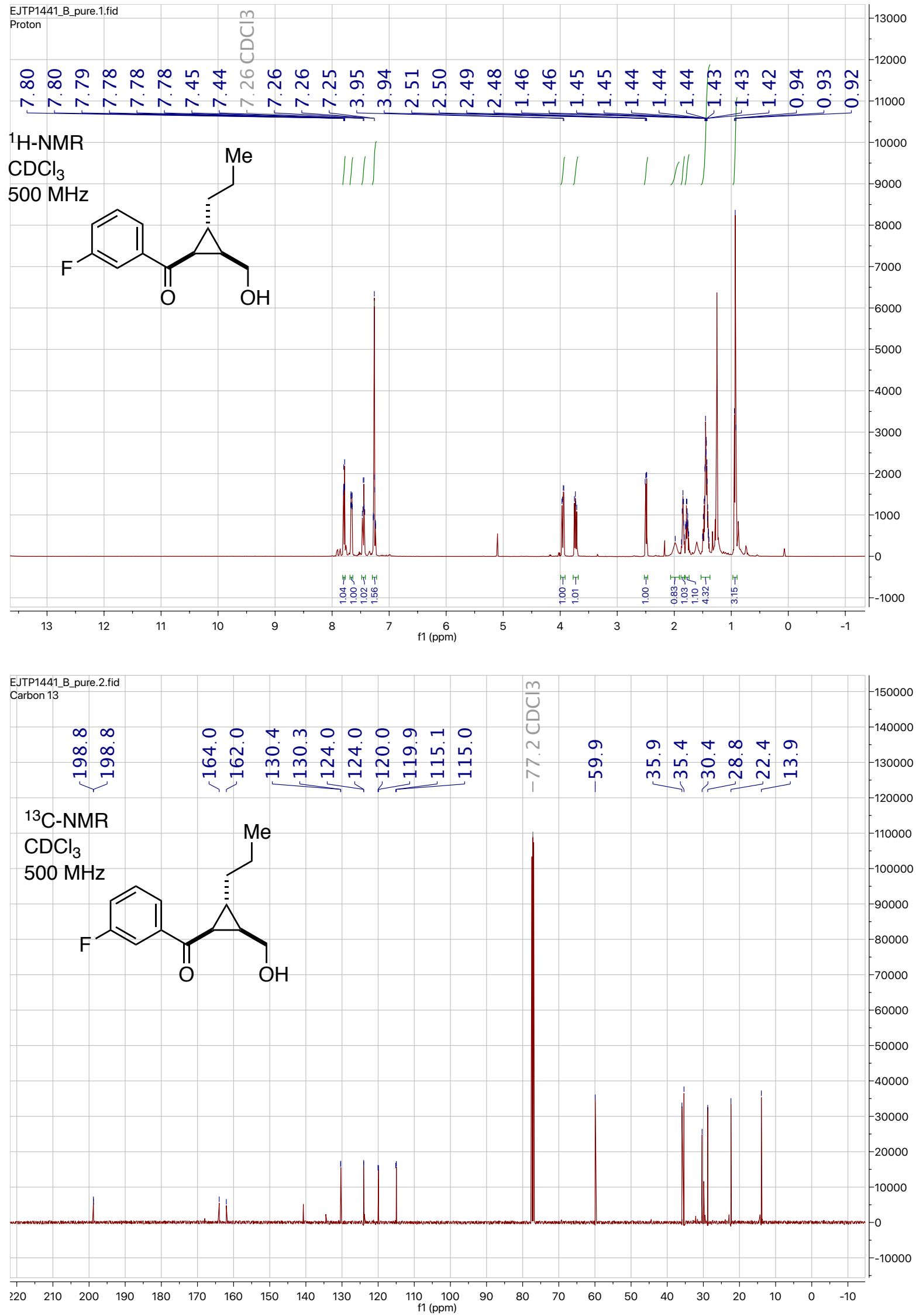


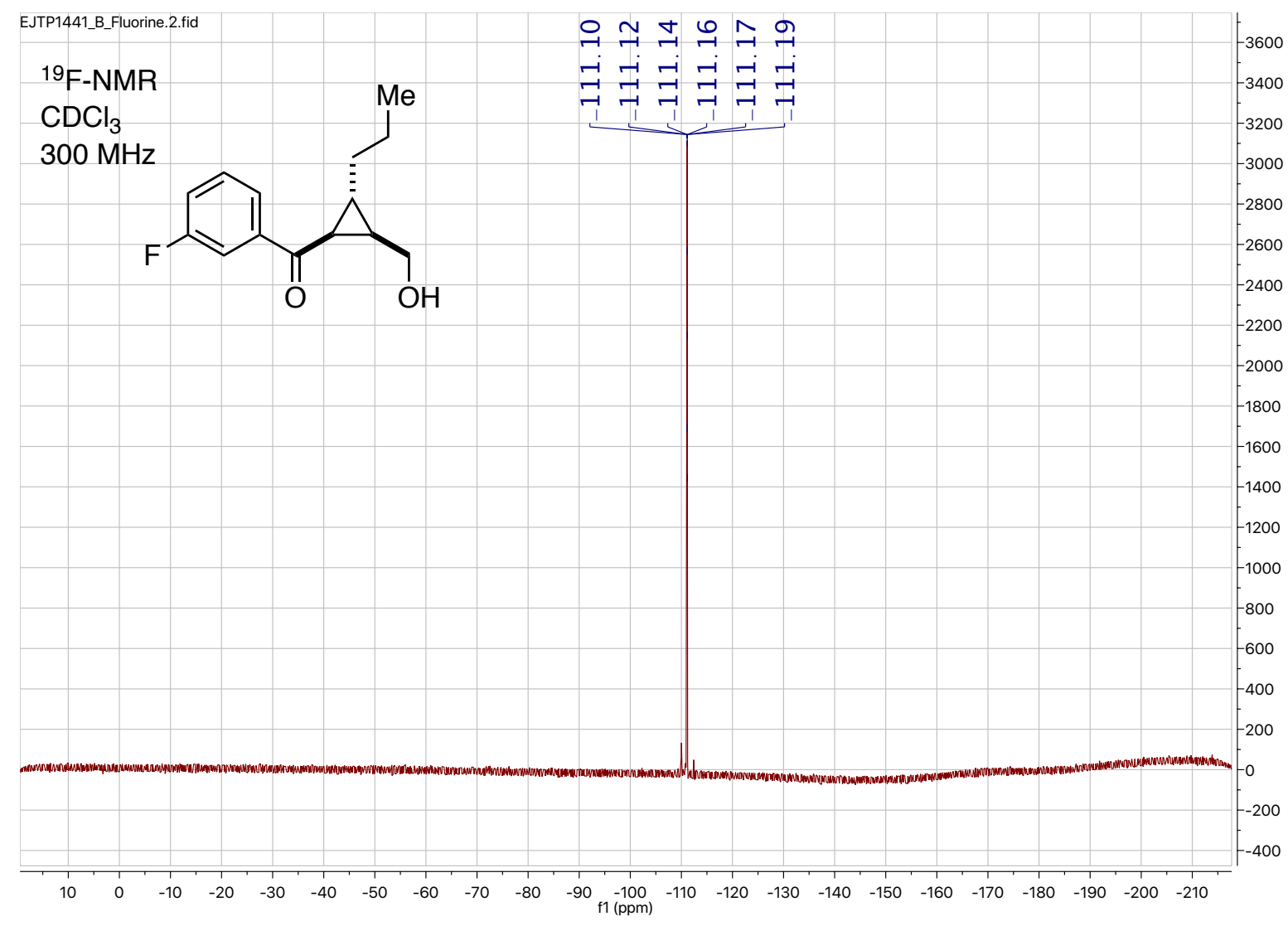



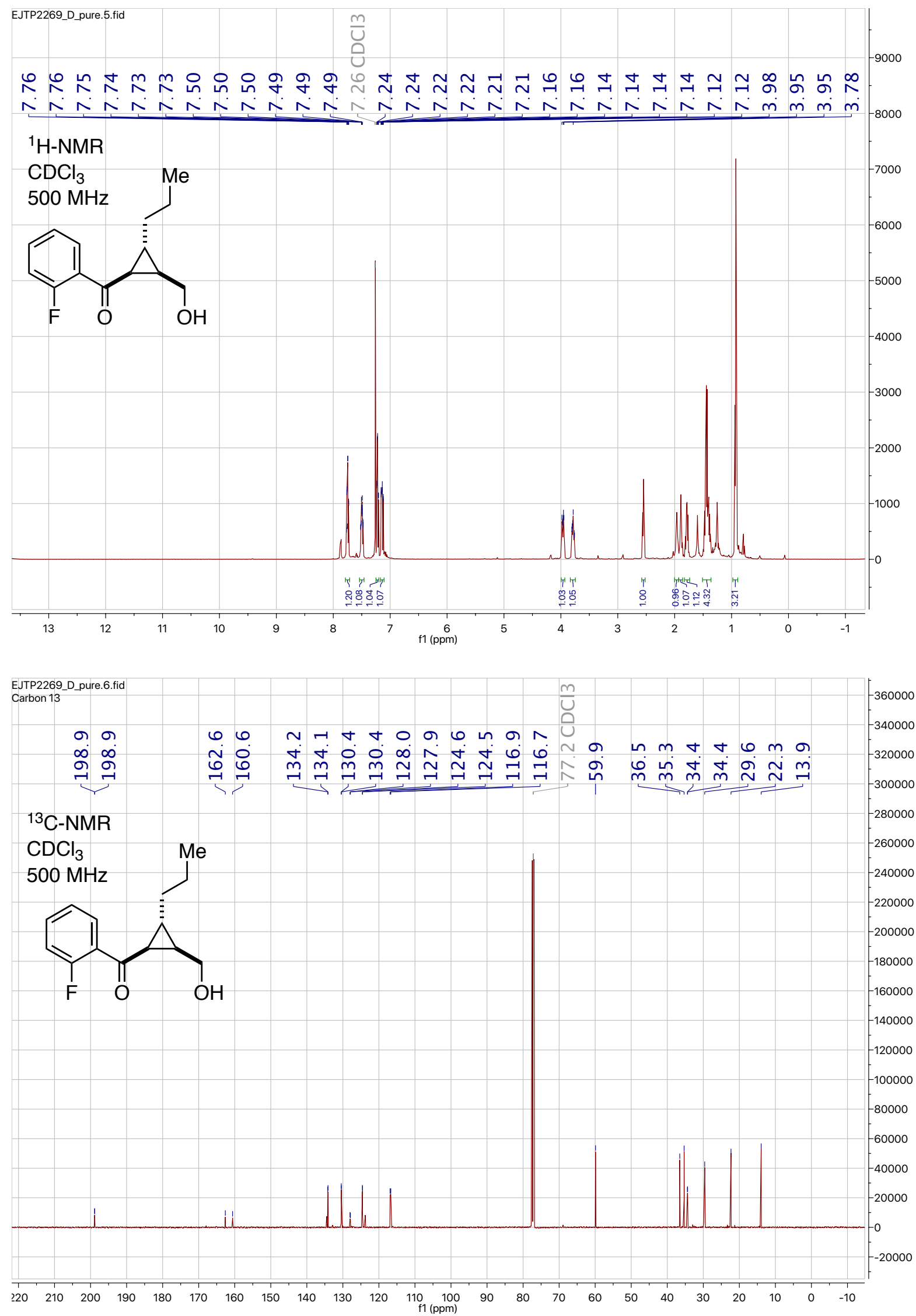


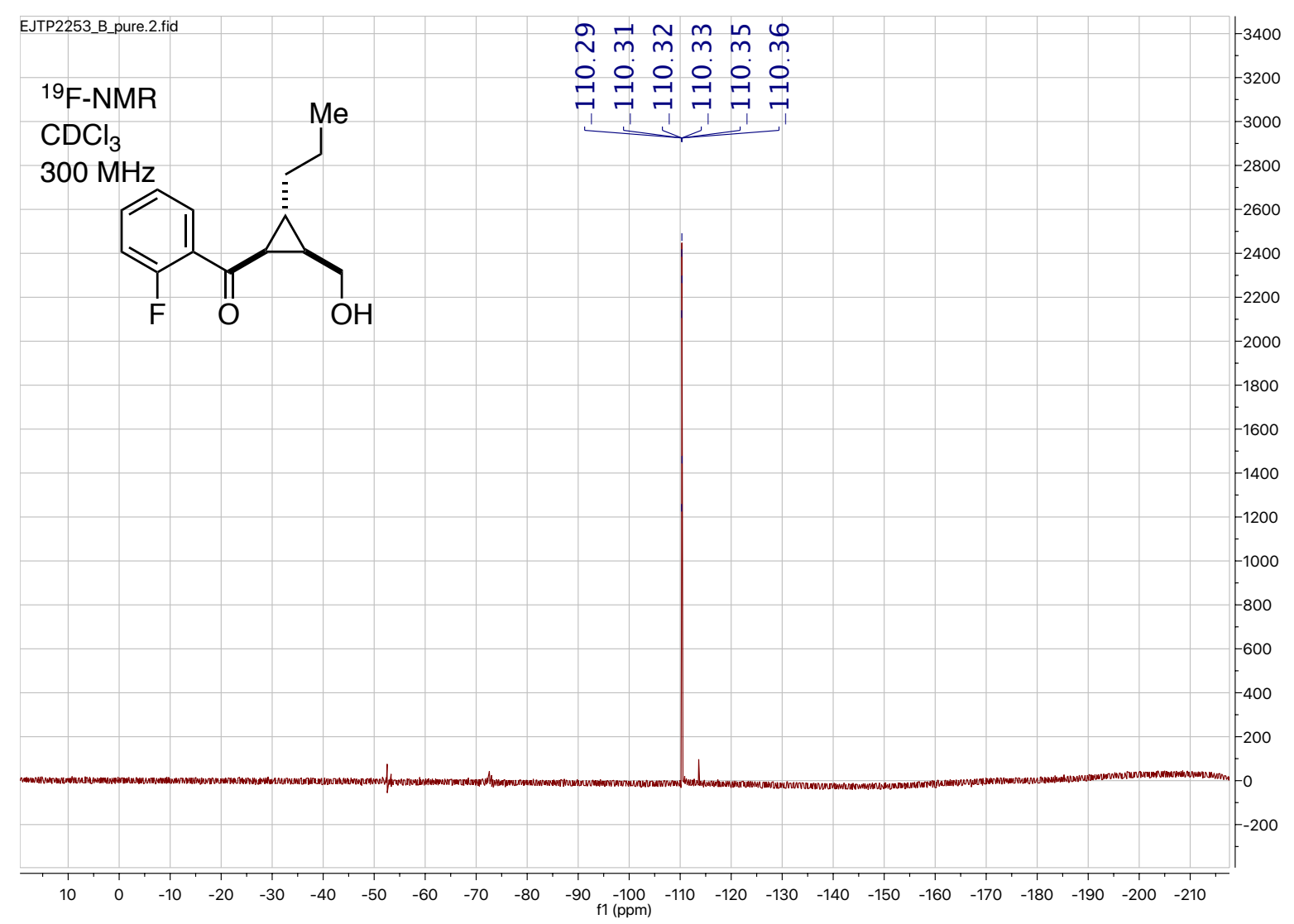



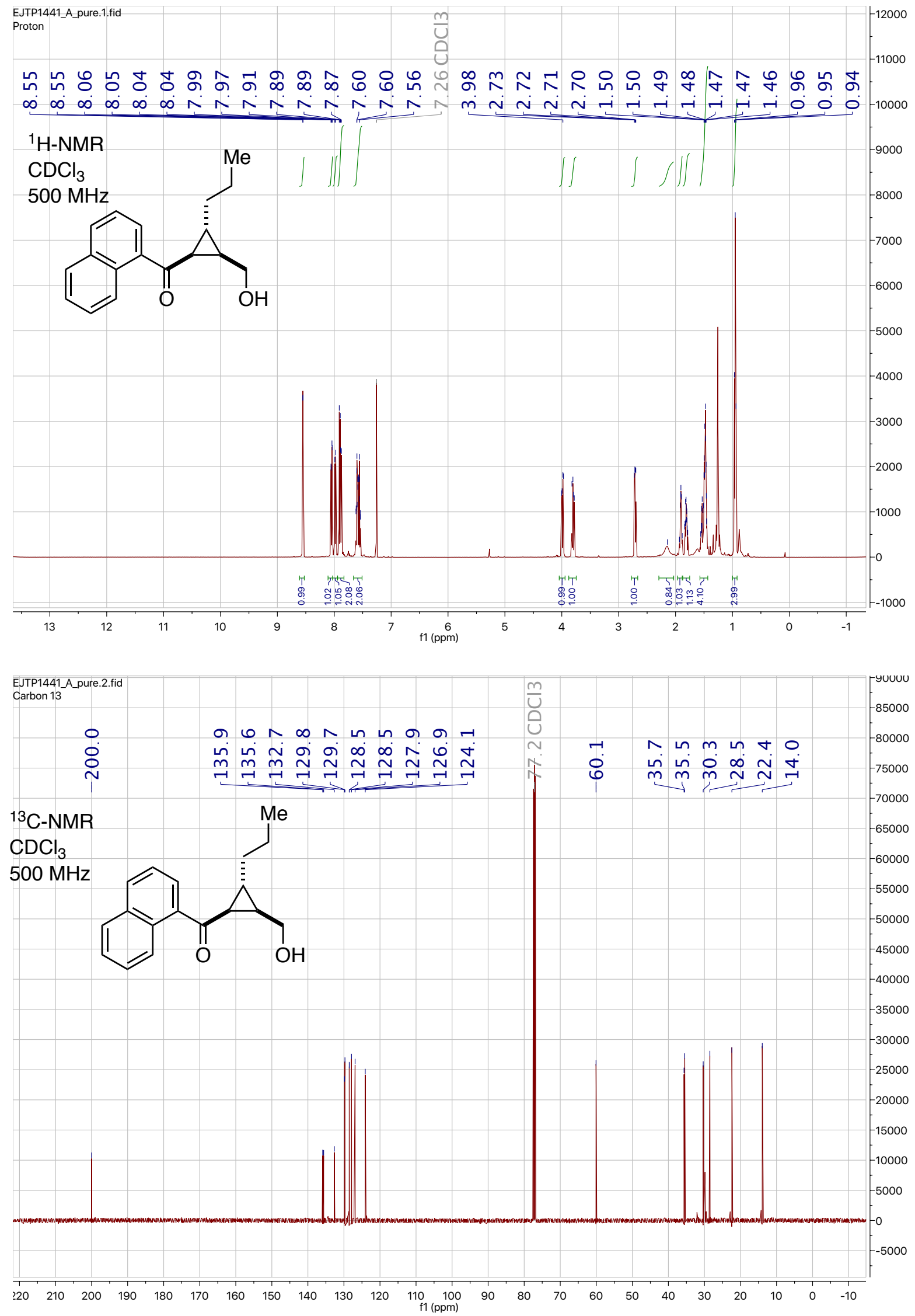

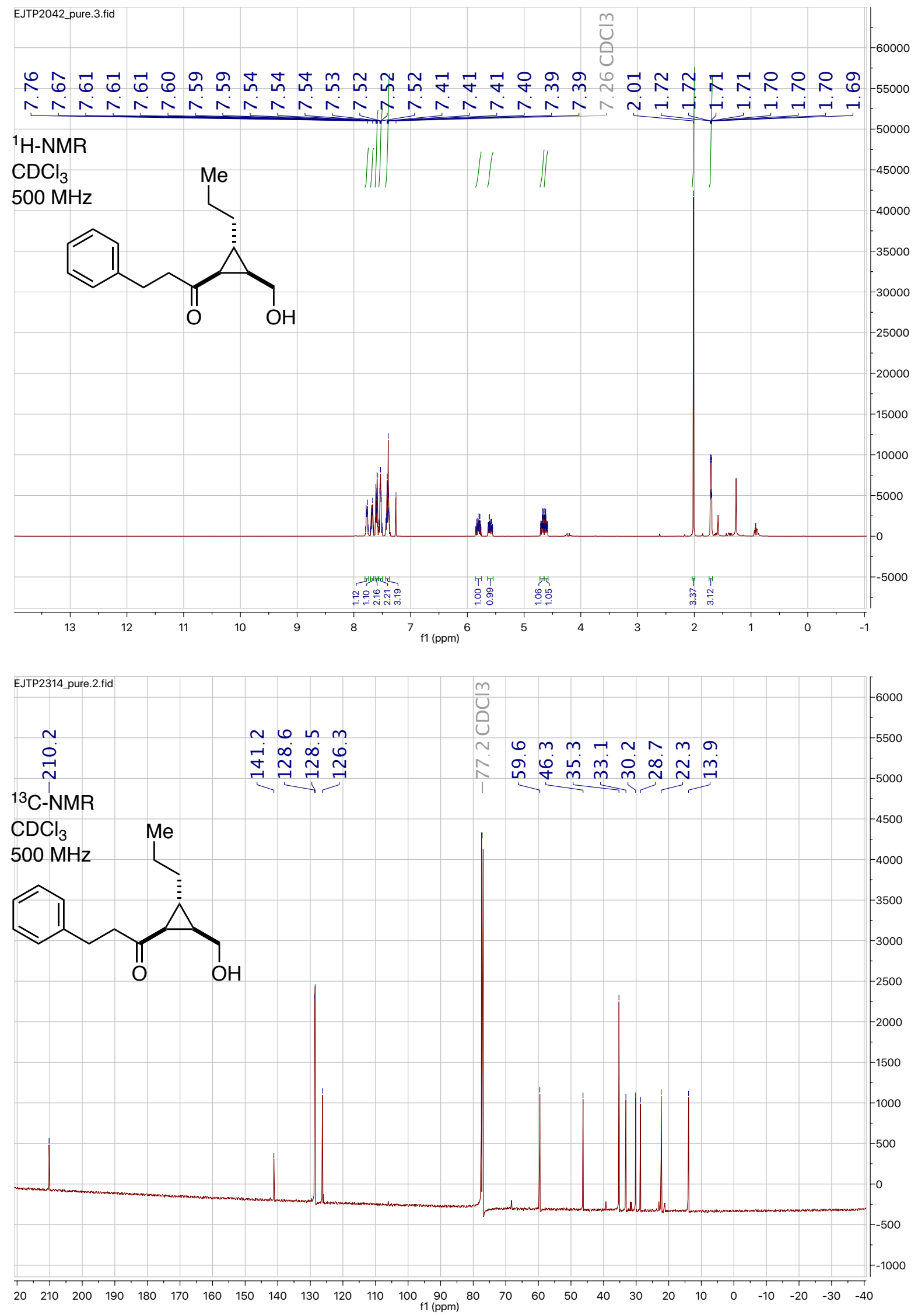

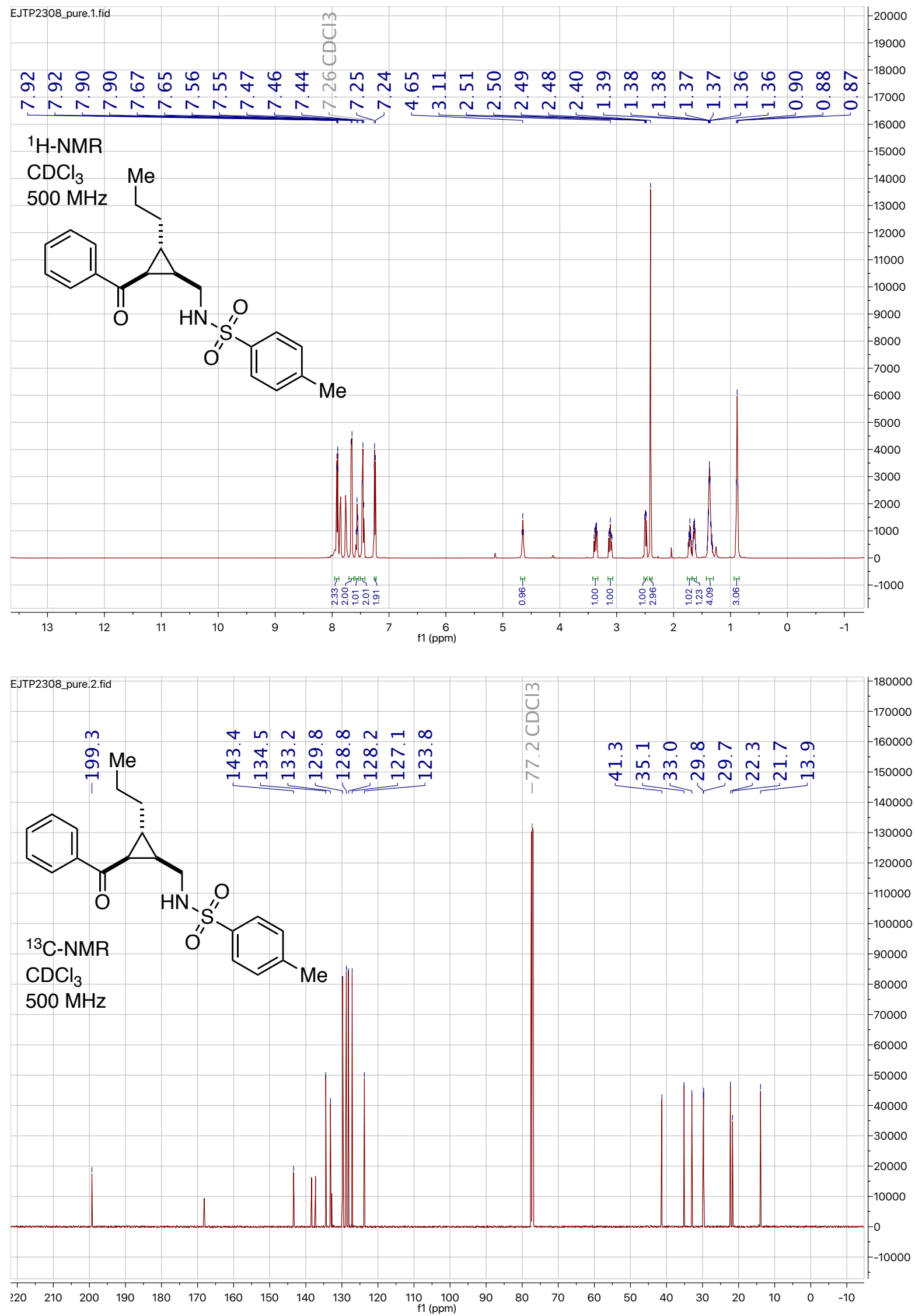

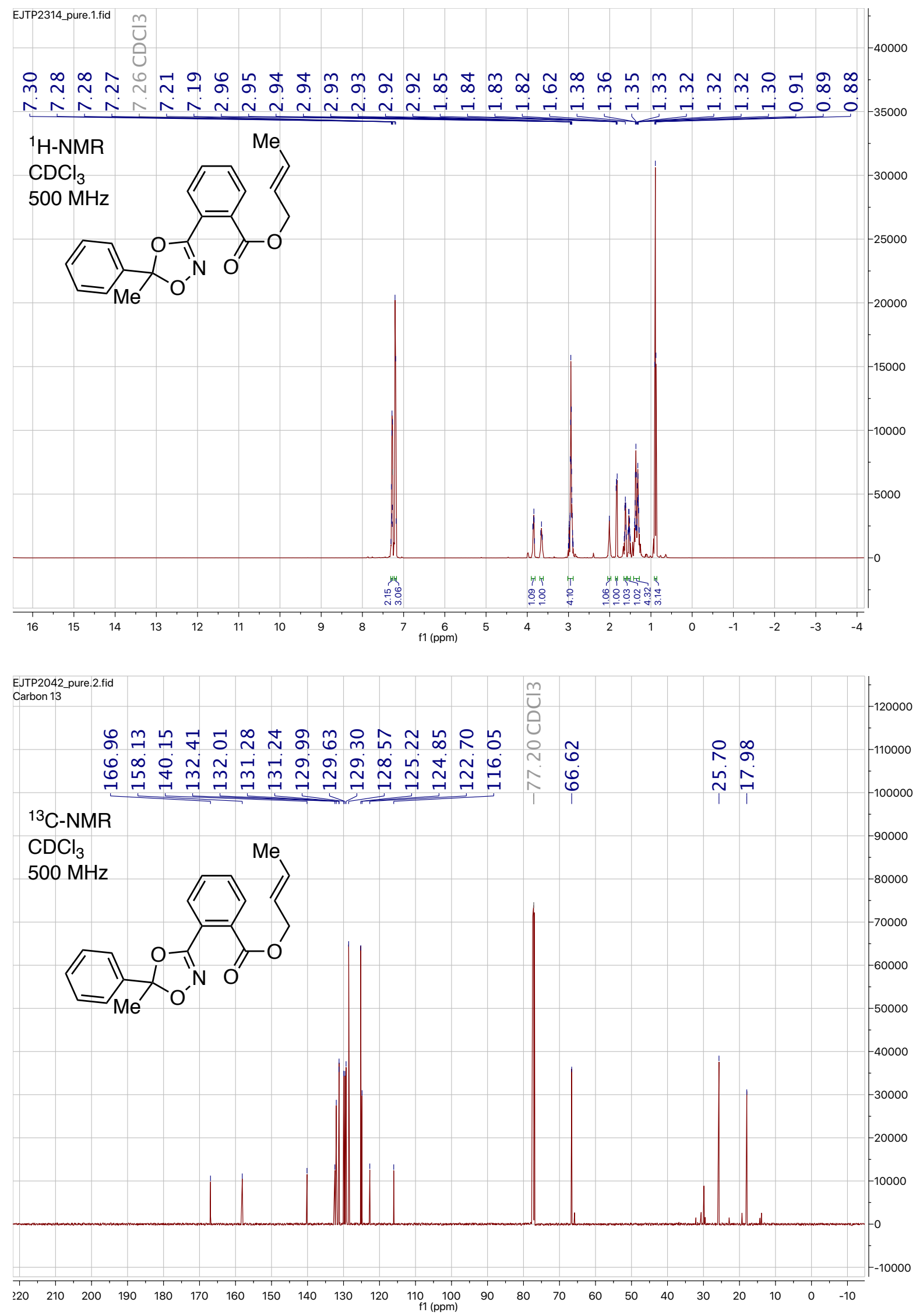

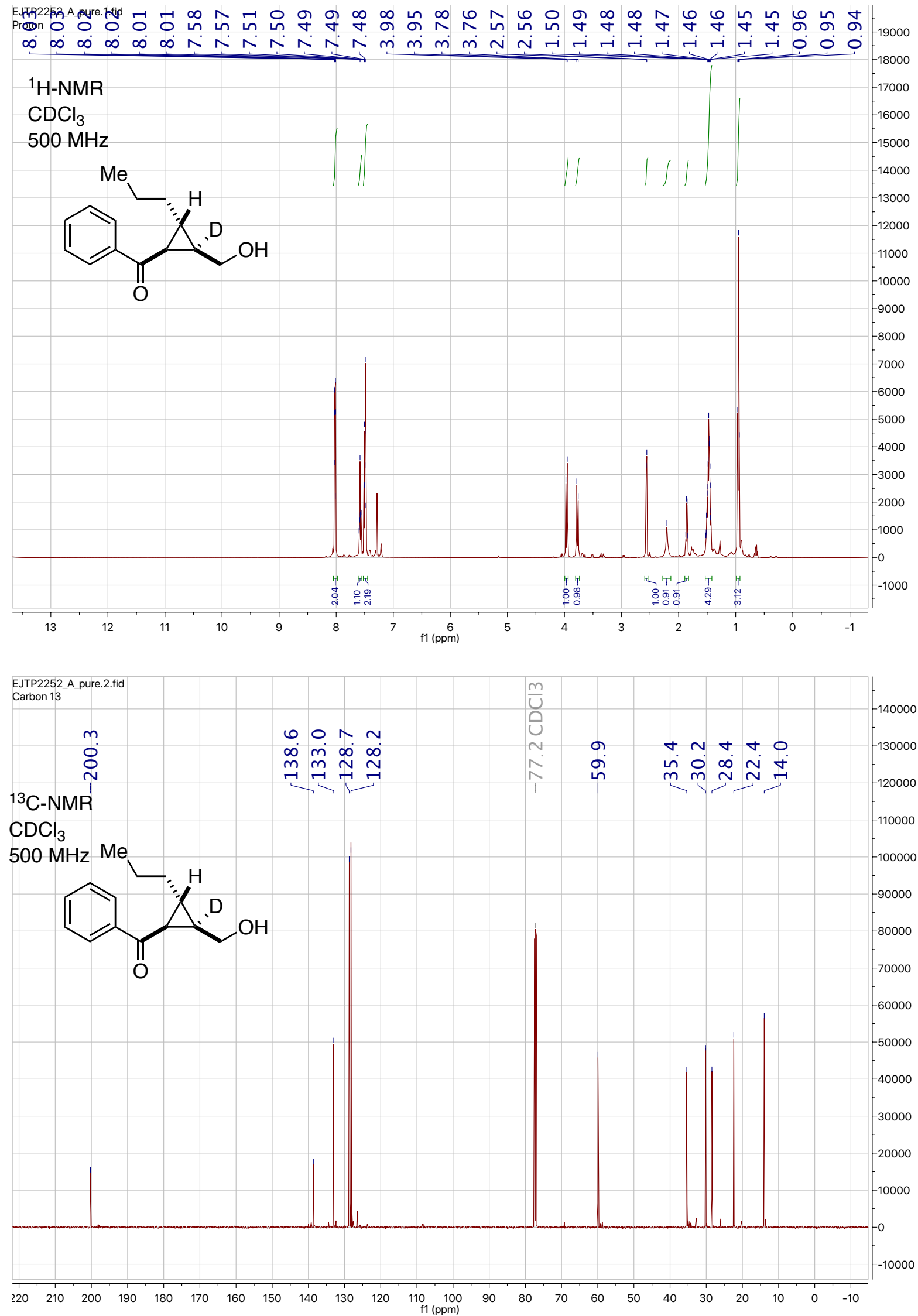


\section{References}

${ }^{1}$ Still, W. C.; Kahn, M.; Mitra, A. J. Org. Chem. 1978, 43, 2923-2925.

${ }^{2}$ Gassman, P.G.; Sowa, J.R. 1,2,3,4-Tetraalkyl-5-perfluoroalkyl-cyclopentadiene, di(perfluoroalkyl)-trialkylcyclopentadiene and transition metal complexes thereof, U.S. Patent 5,245,064, Sep. 14, 1993.

${ }^{3}$ 3a-3j: Piou, T.; Rovis, T. J. Am. Chem. Soc., 2014, 136, 11292.

${ }^{4}$ 3k: Duchemin, C.; Cramer, N. Org. Chem. Front., 2019, 6, 209.

${ }^{5}$ Kim, J.D.; Lee, M.H.; Han, G.; Park, H.; Zee, O.P.; Jung, Y.H. Tetrahedron, 2001, 57, 8257.

${ }^{6}$ 2j: Tasukawa, T.; Miyamura, H.; Kobayashi, S. J. Am. Chem. Soc., 2012, 134, 16963.

${ }^{7}$ 2k: Wonk, K.C.; Ng, E.; Wong, W.-T.; Chiu, P. Chem. Eur. J., 2016, 22, 3709.

${ }^{8}$ Cyclooctenol: Li, J.; Jia, S.; Chen, P. R. Nature Chemical Biology, 2014, 10, 1003.

${ }^{9}$ 2a-d $\boldsymbol{d}_{1}$ : Fox, R.J; Lalic, G; Bergman, R.G. J. Am. Chem. Soc., 2007, 129, 14144.

${ }^{10}$ 8a: Park, S. R.; Kim, C.; Kim, D.; Thrimurtulu, N.; Yeom, H.-S.; Jun, J.; Shin, S.;Rhee, Y.H. Org. Lett., 2013, 15, 1166.

${ }^{11}$ 9a: Motokuni, K; Takeuchi, D.; Osakada, K.; Polym. Chem., 2015, 6, 1248.

${ }^{12}$ (a) Graham, J. D.; Rogers, M. T. J. Am. Chem. Soc. 1962, 84, 2249. (b) Wiberg, K. B.; Nist, B. J. J. Am. Chem. Soc. 1963, 85, 2788.

${ }^{13}$ Blanc, E.; Schwarzenbach, D.; Flack, H. D. J. Appl. Cryst. 24 (1991), 1035-1041.

${ }^{14}$ Clark. R. C.; Reid, J. S. Acta Cryst. A51 (1995), 887-897.

${ }^{15}$ Version 1.171.38.46 (2015). Rigaku Oxford Diffraction.

${ }^{16}$ Sheldrick, G. M. Acta Cryst. A71 (2015), 3-8.

${ }^{17}$ Sheldrick, G. M. Acta Cryst. C71 (2015), 3-8.

${ }^{18}$ Dolomanov, O. V.; Bourhis, L. J.; Gildea, R. J.; Howard, J. A. K.; Puschmann, H. J. Appl. Cryst. 42 (2009), 339-341.

${ }^{19}$ Spek, A. Acta Cryst. D65 (2009), 148-155.

${ }^{20}$ CrystalMaker Software Ltd, Oxford, England (www.crystalmaker.com). 